\title{
INVESTIGATION OF DUAL ACTIVE NUCLEI, OUTFLOWS, SHOCK-HEATED GAS, AND YOUNG STAR CLUSTERS IN MARKARIAN 266
}

\author{
J. M. Mazzarella ${ }^{1}$, K. Iwasawa ${ }^{2}$, T. Vavilkin ${ }^{3}$, L. Armus ${ }^{4}$, D.-C. $\mathrm{KIm}^{5,6}$, G. Bothun ${ }^{7}$, A. S. Evans ${ }^{5,6}$,

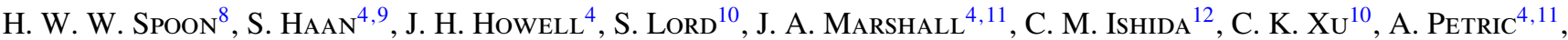 \\ D. B. Sanders ${ }^{13}$, J. A. Surace ${ }^{4}$, P. Appleton ${ }^{10}$, B. H. P. Chan ${ }^{1}$, D. T. FraYer $^{6}$, H. InAmi ${ }^{4}$, E. Ye. Khachikian ${ }^{14}$, \\ B. F. Madore ${ }^{1,15}$, G. C. Privon ${ }^{5}$, E. Sturm ${ }^{16}$, Vivian $U^{13}$, and S. Veilleux ${ }^{17}$ \\ ${ }^{1}$ Infrared Processing \& Analysis Center, MS 100-22, California Institute of Technology, \\ Pasadena, CA 91125, USA; mazz@ipac.caltech.edu, bchan@ipac.caltech.edu \\ 2 ICREA and Institut del Ciències del Cosmos (ICC), Universitat de Barcelona (IEEC-UB), Martí i Franquès 1, \\ E-08028 Barcelona, Spain; kazushi.iwasawa@icc.ub.edu \\ ${ }^{3}$ Department of Physics and Astronomy, State University of New York at Stony Brook, \\ Stony Brook, NY 11794-3800, USA; vavilkin@ grad.physics.sunysb.edu \\ ${ }^{4}$ Spitzer Science Center, MS 314-6, California Institute of Technology, Pasadena, CA 91125, USA; \\ lee@ipac.caltech.edu, jhhowell@ipac.caltech.edu, jason@ipac.caltech.edu, inami@ipac.caltech.edu, haan@ipac.caltech.edu \\ ${ }^{5}$ Department of Astronomy, University of Virginia, Charlottesville, VA 22904-4325, USA; aevans@ virginia.edu, gcp8y@ virginia.edu \\ ${ }^{6}$ National Radio Astronomy Observatory, Charlottesville, VA 22903-2475, USA; dkim@nrao.edu, dfrayer@nrao.edu \\ ${ }^{7}$ Physics Department, University of Oregon, Eugene, OR 97402, USA; nuts@ @igmoo.uoregon.edu \\ ${ }^{8}$ Department of Astronomy, Cornell University, Ithaca, NY 14853, USA; spoon@ isc.astro.cornell.edu \\ ${ }^{9}$ CSIRO Astronomy \& Space Science, ATNF, Marsfield NSW 2122, Australia; sebastian.haan@ @ csiro.au \\ ${ }^{10}$ NASA Herschel Science Center, MS 100-22, California Institute of Technology, Pasadena, CA 91125, USA; \\ lord@ipac.caltech.edu, apple@ipac.caltech.edu, cxu@ipac.caltech.edu \\ ${ }^{11}$ Astronomy Department, California Institute of Technology, MS 249-17, Pasadena, CA 91125, USA \\ jason.mashall@caltech.edu,ap@astro.caltech.edu \\ ${ }^{12}$ Subaru Telescope, National Astronomical Observatory of Japan, Hilo, HI 96720, USA; cmishida@mac.com \\ ${ }^{13}$ Institute for Astronomy, University of Hawaii, Honolulu, HI 96822, USA; sanders@ifa.hawaii.edu, vivian@ifa.hawaii.edu \\ ${ }^{14}$ Byurakan Astrophysical Observatory, Academician of National Academy of Sciences of Armenia, Byurakan, \\ 378433 Aragatsodn Province, Republic of Armenia; khache@bao.sci.am \\ 15 The Observatories, Carnegie Institution of Washington, Pasadena, CA 91101, USA; barry@obs.carnegiescience.edu \\ ${ }^{16}$ Max-Planck-Institut für extraterrestrische Physik, Postfach 1312, D-85741 Garching, Germany; sturm@mpe.mpg.de \\ ${ }^{17}$ Department of Astronomy, University of Maryland, College Park, MD 20742, USA; veilleux@ astro.umd.edu \\ Received 2010 November 13; accepted 2012 July 29; published 2012 September 24
}

\begin{abstract}
Results of observations with the Spitzer, Hubble, GALEX, Chandra, and XMM-Newton space telescopes are presented for the luminous infrared galaxy (LIRG) merger Markarian 266. The SW (Seyfert 2) and NE (LINER) nuclei reside in galaxies with Hubble types $\mathrm{SBb}$ (pec) and S0/a (pec), respectively. Both companions are more luminous than $L^{*}$ galaxies and they are inferred to each contain a $\approx 2.5 \times 10^{8} M_{\odot}$ black hole. Although the nuclei have an observed hard X-ray flux ratio of $f_{X}(N E) / f_{X}(S W)=6.4$, Mrk $266 \mathrm{SW}$ is likely the primary source of a bright $\mathrm{Fe} \mathrm{K} \alpha$ line detected from the system, consistent with the reflection-dominated X-ray spectrum of a heavily obscured active galactic nucleus (AGN). Optical knots embedded in an arc with aligned radio continuum radiation, combined with luminous $\mathrm{H}_{2}$ line emission, provide evidence for a radiative bow shock in an AGN-driven outflow surrounding the NE nucleus. A soft X-ray emission feature modeled as shock-heated plasma with $T \sim 10^{7} \mathrm{~K}$ is cospatial with radio continuum emission between the galaxies. Mid-infrared diagnostics provide mixed results, but overall suggest a composite system with roughly equal contributions of AGN and starburst radiation powering the bolometric luminosity. Approximately 120 star clusters have been detected, with most having estimated ages less than 50 Myr. Detection of $24 \mu \mathrm{m}$ emission aligned with soft X-rays, radio continuum, and ionized gas emission extending $\sim 34^{\prime \prime}\left(20 \mathrm{kpc}\right.$ ) north of the galaxies is interpreted as $\sim 2 \times 10^{7} M_{\odot}$ of dust entrained in an outflowing superwind. At optical wavelengths this Northern Loop region is resolved into a fragmented morphology indicative of Rayleigh-Taylor instabilities in an expanding shell of ionized gas. Mrk 266 demonstrates that the dust "blowout" phase can begin in a LIRG well before the galaxies fully coalesce during a subsequent ultraluminous infrared galaxy (ULIRG) phase, and rapid gas consumption in luminous dual AGNs with kiloparsec-scale separations early in the merger process may explain the paucity of detected binary QSOs (with parsec-scale orbital separations) in spectroscopic surveys. An evolutionary sequence is proposed representing a progression from dual to binary AGNs, accompanied by an increase in observed $L_{x} / L_{\text {ir }}$ ratios by over two orders of magnitude.
\end{abstract}

Key words: galaxies: active - galaxies: interactions - galaxies: nuclei - galaxies: Seyfert - galaxies: starburst galaxies: star clusters: general

Online-only material: color figures

\section{INTRODUCTION}

\subsection{LIRGs, ULIRGs, and GOALS}

Luminous infrared galaxies (LIRGs; $10^{11} L_{\odot} \leqslant L_{\text {ir }}<$ $10^{12} L_{\odot}$ ) and ultraluminous infrared galaxies (ULIRGs; $L_{\mathrm{ir}} \geqslant$
$10^{12} L_{\odot}$ ) are intriguing objects with widespread implications for galaxy evolution. They contain the highest known rates of star formation, they exhibit a high frequency of active galactic nuclei (AGNs) and large-scale outflows (superwinds), and mounting evidence indicates that the majority of local (U)LIRGs represent 
an evolutionary process involving the transformation of major mergers between dusty, gas-rich disk galaxies into elliptical galaxies hosting classical UV-excess QSOs or powerful radio galaxies (e.g., Sanders \& Mirabel 1996; Veilleux 2006). At redshifts of $z \sim 1$, LIRGs have a higher space density than ULIRGs and dominate the total star formation density at that epoch (Le Floc'h et al. 2005). At $z \sim 2$ the contributions of LIRGs and ULIRGs to the total IR luminosity density are about equal (e.g., Caputi et al. 2007). Since LIRGs and ULIRGs were much more common in the early universe, these populations are fundamental in understanding both star formation and galaxy evolution.

The Great Observatories All-Sky LIRG Survey (GOALS) ${ }^{18}$ is utilizing imaging and spectroscopic data from NASA's Spitzer, Hubble, Chandra, and GALEX space observatories, in combination with ground-based observations, in a comprehensive study of more than 200 of the most luminous infrared-selected galaxies in the local universe (Armus et al. 2009). The sample consists of 181 LIRGs and 23 ULIRGs that form a statistically complete subset of the fluxlimited IRAS Revised Bright Galaxy Sample (RBGS), which itself is composed of 629 extragalactic objects with $60 \mu \mathrm{m}$ flux densities above 5.24 Jy and Galactic latitudes $|b|>5^{\circ}$ (Sanders et al. 2003).

\subsection{Dual AGNs in Major Mergers}

A key scientific driver for GOALS is the investigation of the relative timescales and energetics of active star formation and AGN phenomena during different phases of the merger sequence. It is now widely accepted that all massive galaxies likely have a supermassive black hole $(\mathrm{SMBH})$ in their centers (e.g., Ferrarese \& Ford 2005), with a SMBH mass proportional to the mass of the stellar bulge $\left(M_{\mathrm{SMBH}} / M_{\text {bulge }} \approx 0.2 \%\right.$; Marconi \& Hunt 2003). However, many uncertainties remain regarding the fueling of paired SMBHs during major mergers. While the nuclei are still separated by kiloparsec scales, how common is it for both SMBHs to have accretion rates high enough to produce energetically significant dual AGNs, as opposed to one or both nuclei being powered predominantly by a burst of star formation? How do AGN characteristics depend on properties of the host galaxies and dynamics of the encounter? Are the fuel supplies and accretion rates sufficient to sustain two luminous AGNs well into a true binary stage (e.g., binary QSOs), when the SMBHs are closely bound $(r<100 \mathrm{pc})$ in Keplerian orbits inside a dynamically relaxed (elliptical) merger remnant (e.g., Colpi \& Dotti 2009)? Is there something special about the physical conditions in systems that host dual AGNs, or is their lack of detection in some (U)LIRGs merely a matter of observing them during the right stage prior to merging (i.e., an evolutionary timing coincidence), or accounting for differences in nuclear dust obscuration? What is the relative importance of AGNs and star formation in the energetics of the "superwind" phenomenon that appears to be ubiquitous in (U)LIRGs (e.g., see the review by Veilleux 2006)?

Although (U)LIRGs are predominantly systems involving major mergers, extensive observations utilizing optical and infrared spectroscopy have so far turned up surprisingly few systems containing confirmed dual AGNs. This has a number of possible explanations. First, (U)LIRGs contain large quantities of centrally concentrated dust that can effectively obscure one or both AGNs at optical, near-infrared, and even midinfrared wavelengths (e.g., Laurent et al. 2000; Spoon et al.

\footnotetext{
18 http://goals.ipac.caltech.edu/
}

2004). Therefore, circumnuclear star formation can dominate the observed spectra while in reality a powerful AGN may be buried inside. Second, about $30 \%$ of observed LIRGs (e.g., Veilleux et al. 1995) and $~ 40 \%$ of ULIRGs (e.g., Veilleux et al. 1999) are classified as LINERs based on optical spectroscopy. It has proven difficult to distinguish between low-luminosity AGNs (photoionization from radiation due to accretion onto a SMBH), photoionization from very hot stars, and shock heating (induced by supernovae ( $\mathrm{SNe}$ ) and starburst-driven superwinds) as the primary source of ionization in LINERs (e.g., Veilleux et al. 1999).

Recent results indicate that most nearby LINERs are dominated by photoionization rather than shock heating, and that they are an important class of AGNs distinguished primarily by a lower accretion rate than in Seyfert nuclei (Kewley et al. 2006; Ho 2008). This implies that the frequency of dual AGNs may be much higher than inferred to date. Only recently, with the capability of the Chandra X-ray Observatory to penetrate their extensive dust cocoons with high-resolution hard X-ray observations, have suspected dual AGNs been confirmed in a small number of (U)LIRGs. Perhaps the best-known example is NGC 6240, in which hard X-rays and strong $\mathrm{Fe} \mathrm{K} \alpha$ emission lines indicate the presence of two AGNs with a projected separation of 1".4 (1 kpc) (Komossa et al. 2003). A second case is the ULIRG Mrk 463, a system first pointed out as an active double-nucleus galaxy by Petrosian et al. (1978). The eastern component undoubtedly hosts a dust-enshrouded Type 1 AGN that is powering apparently young radio jets (Mazzarella et al. 1991b), but conflicting optical spectral classifications from various studies left the nature of the western nucleus in doubt (LINER, Seyfert 2, or starburst/AGN composite). Dual AGNs in Mrk 463 have been confirmed recently via detection of two compact, luminous sources of hard X-rays using Chandra (Bianchi et al. 2008). To date, very few systems in the GOALS sample have been confirmed to contain dual AGNs via X-ray observations. The first system is NGC 6240 and the second is Arp 299 (NGC 3690 + IC 694) (Ballo et al. 2004). This article presents an in-depth investigation of a third system in this rare class of objects that has considerable importance for understanding the evolution of galaxy mergers and their remnants.

\subsection{Complex Phenomena in Mrk 266}

One of the most remarkable LIRGs in the local universe is Mrk 266 (NGC 5256). The system was first called out as an extraordinary object in the Markarian Survey (First Byurakan Sky Survey) of ultraviolet-excess galaxies due to the presence of two nuclei within what appeared to be a single galaxy (Petrosian et al. 1978, 1979). In a detailed spectroscopic investigation at optical wavelengths, Petrosian et al. (1980) showed that the nuclei have a radial velocity difference of $280 \mathrm{~km} \mathrm{~s}^{-1}$, and based on the assumption that the nuclei revolve about a common center of gravity, masses of $7 \times 10^{9}$ and $10 \times 10^{9} M_{\odot}$ were estimated for the SW and NE nuclei, respectively. As for all objects in the Markarian Survey, Mrk 266 stood out because its UV-to-optical flux ratio is higher than in normal galaxies. However, Mrk 266 is also a LIRG, with an infrared luminosity of $L_{\mathrm{ir}}=3.6 \times 10^{11} L_{\odot}(8-1000 \mu \mathrm{m}$, as estimated from flux densities in all four IRAS bands; Armus et al. 2009). As shown in the current study (Section 3.5), the global spectral energy distribution (SED) indicates that the bulk of its energy is emitted in the mid- to far-infrared; much more energy is being emitted at the peak of the SED than is escaping in UV radiation $\left(\nu L_{v}(70 \mu \mathrm{m}) / \nu L_{v}(0.2 \mu \mathrm{m}) \approx 20\right)$. 
Mrk 266 contains a filamentary nebula of ionized hydrogen $\sim 30 \mathrm{kpc}$ in diameter (Armus et al. 1990) surrounding the two nuclei. A luminous X-ray nebula $\sim 100 \mathrm{kpc}$ in extent detected by ROSAT (also surrounding the two nuclei) and complex kinematics derived from optical spectroscopy revealed one of the most spectacular examples of an outflowing, starburstdriven superwind (Wang et al. 1997). In addition to radio continuum emission from the two nuclei, a bright radio source located between the nuclei was detected and interpreted as enhanced synchrotron emission induced by extensive shocks at the interface of the merging galaxies (Mazzarella et al. 1988). Imaging in $\mathrm{H} \alpha+\left[\mathrm{N}_{\mathrm{II}}\right]$ (Mazzarella et al. 1988; Armus et al. 1990), [O III] $\lambda 5007$ (Hutchings et al. 1988), and soft X-rays (Kollatschny \& Kowatsch 1998) also revealed a bright loop of emission extending $\sim 24^{\prime \prime}(17 \mathrm{kpc})$ to the northeast and connected to the SW (Seyfert 2) nucleus (Wang et al. 1997). Recent Chandra observations have resolved X-ray emission from both nuclei, and detected diffuse emission associated with the northern feature and between the nuclei (Brassington et al. 2007). The nature of the northern emission region (hereafter called the Northern Loop) has remained controversial, with suggestions including a component of the superwind (Wang et al. 1997), a "jet" from an AGN (Kollatschny \& Kowatsch 1998), and a tidal tail (Brassington et al. 2007).

Mrk 266 is worthy of further detailed study because it manifests complex processes that are rarely detected simultaneously, presumably due to their relatively short time scales: vigorous star formation, a candidate dual AGN (depending on the ionizing source of the LINER), a powerful large-scale superwind, and substantial radio continuum and X-ray emission between the nuclei. The relative proximity of the system permits closeup investigation of an important stage in the evolution of major mergers that may involve the transformation of LIRGs into ULIRGs, AGN feedback with the interstellar medium (ISM), and implications for fueling (or quenching) of accreting SMBHs which galaxy evolution models predict to have occurred in large numbers during earlier epochs.

In this study, new observations and re-processed archival data from the Spitzer, Hubble, Chandra, XMM-Newton, and GALEX space telescopes are analyzed in combination with multi-wavelength ground-based data. Imaging, photometric, and spectroscopic data are presented in Section 2. In Section 3, the new data are interpreted to obtain new insights into the nature of the colliding/merging system (Section 3.1), the nuclei and circumnuclear regions (Section 3.2), the region between the nuclei (Section 3.3), the extensive superwind (Section 3.4), the SEDs of the major components (Section 3.5), newly detected star clusters (SCs, Section 3.6), and the molecular gas properties (Section 3.7). In Section 3.8, Mrk 266 is examined in comparison with other interacting systems with strong radiation sources between the galaxies, and a sequence is proposed that may represent an evolutionary progression from dual AGNs (with kiloparsec-scale separations) to binary AGNs (with parsec-scale orbits). Due to the large number of new measurements spanning many spectral regions, the basic observations and data reductions are described in Section 2, but most of the corresponding figures and tables are displayed alongside their interpretation and analyses in Section 3. Conclusions are summarized in Section 4. A systemic heliocentric recession velocity of $8353 \pm 13 \mathrm{~km} \mathrm{~s}^{-1}$ (de Vaucouleurs et al. 1991) is adopted, corrected to $8825 \pm 22 \mathrm{~km} \mathrm{~s}^{-1}$ via the flow model of Mould et al. (2000a, 2000b) that accounts for three major attractors in the local universe. We adapt cosmological param- eters $H_{\mathrm{o}}=70 \mathrm{~km} \mathrm{~s}^{-1} \mathrm{Mpc}^{-1}, \Omega_{\mathrm{M}}=0.28$, and $\Omega_{\mathrm{V}}=0.72$ (Hinshaw et al. 2009). The corresponding luminosity distance to Mrk 266 is $129 \mathrm{Mpc}$ (distance modulus $35.55 \mathrm{mag}$ ), and the spatial scale is $0.59 \mathrm{kpc} \operatorname{arcsec}^{-1} .19$

\section{OBSERVATIONS}

\subsection{Optical and Near-infrared Imaging with HST}

Mrk 266 was observed on 2005 November 17 with the Advanced Camera for Surveys (ACS) Wide Field Channel (WFC) as part of a Hubble Space Telescope (HST) Cycle 14 GOALS program to investigate 88 LIRGs with $L_{\mathrm{ir}}>10^{11.4} L_{\odot}$ (PID 10592, PI: A. Evans). The system was imaged with an integration time of 22.5 minutes in the F435W filter ( $B$ band, $0.435 \mu \mathrm{m}$, image J9CV48010) and 13 minutes in the F814W filter ( $I$ band, $0.814 \mu \mathrm{m}$, image J9CV48020). The WFC has a field of view of $202^{\prime \prime} \times 202^{\prime \prime}$ with pixels subtending $00^{\prime} 05$. The images were processed through the standard STScI pipeline to remove instrumental signatures, subtract a dark frame, apply flat fielding, remove geometric distortion, and apply flux calibration. Residual cosmic-ray removal, background subtraction, and accurate astrometric calibration using sources from 2MASS (Skrutskie et al. 2006) were also performed using procedures detailed by A. S. Evans et al. (2012, in preparation). Figure 1 presents a composite color image constructed using the $B$ - and $I$-band data, and labels identify major features that are analyzed throughout this article.

Near-infrared observations at $1.6 \mu \mathrm{m}$ (F160W, $H$ band) with the NIC2 aperture of NICMOS were acquired on 1997 September 13 in Cycle 7 (PID 7328, PI: M. Malkan). The image (N44B40010) was retrieved from the HST archive, corrected for bad pixels, cosmic rays were removed, and astrometric calibration was applied by aligning the galaxies with the ACS images. The central regions of the SW and NE galaxies in these three HST band are displayed in context with their analysis in Section 3.1 .

\subsection{Deep $B+V+I$ Band Ground-based Imaging}

To complement the HST high-resolution imagery and reveal the faintest emission possible, Figure 2 shows the result of spatial registration and stacking of images in the $B, V$, and $I$ bands obtained on 1998 December 22 and 2000 May 4 with the University of Hawaii $2.2 \mathrm{~m}$ telescope (effective seeing $\sim 1$ 1.5 FWHM). Details of the data acquisition and reduction are given by Ishida (2004). The data are presented using Gaussian equalization to contrast the low and high surface brightness features in the same view. Highly asymmetric, low surface brightness emission can be seen spanning $\approx 103 \mathrm{kpc}(2.9)$ from the SE to NW extremities, which is approximately twice the extent of the faintest emission visible in the HST ACS data, SDSS, and previously published imagery.

\subsection{X-Ray Observations with Chandra and XMM-Newton}

\subsubsection{Chandra Observations}

Chandra observations were obtained with the Advanced CCD Imaging Spectrometer (ACIS-S) on 2001 November 2 (PI: A. Read) with a total integration time of $19.95 \mathrm{ks}$. The spatial resolution is $\sim 1^{\prime \prime}$ FWHM. These observations were first presented by Brassington et al. (2007). In order to make refined measurements, highlight additional features, and analyze the

\footnotetext{
19 Provided by NED at http://ned.ipac.caltech.edu/.
} 


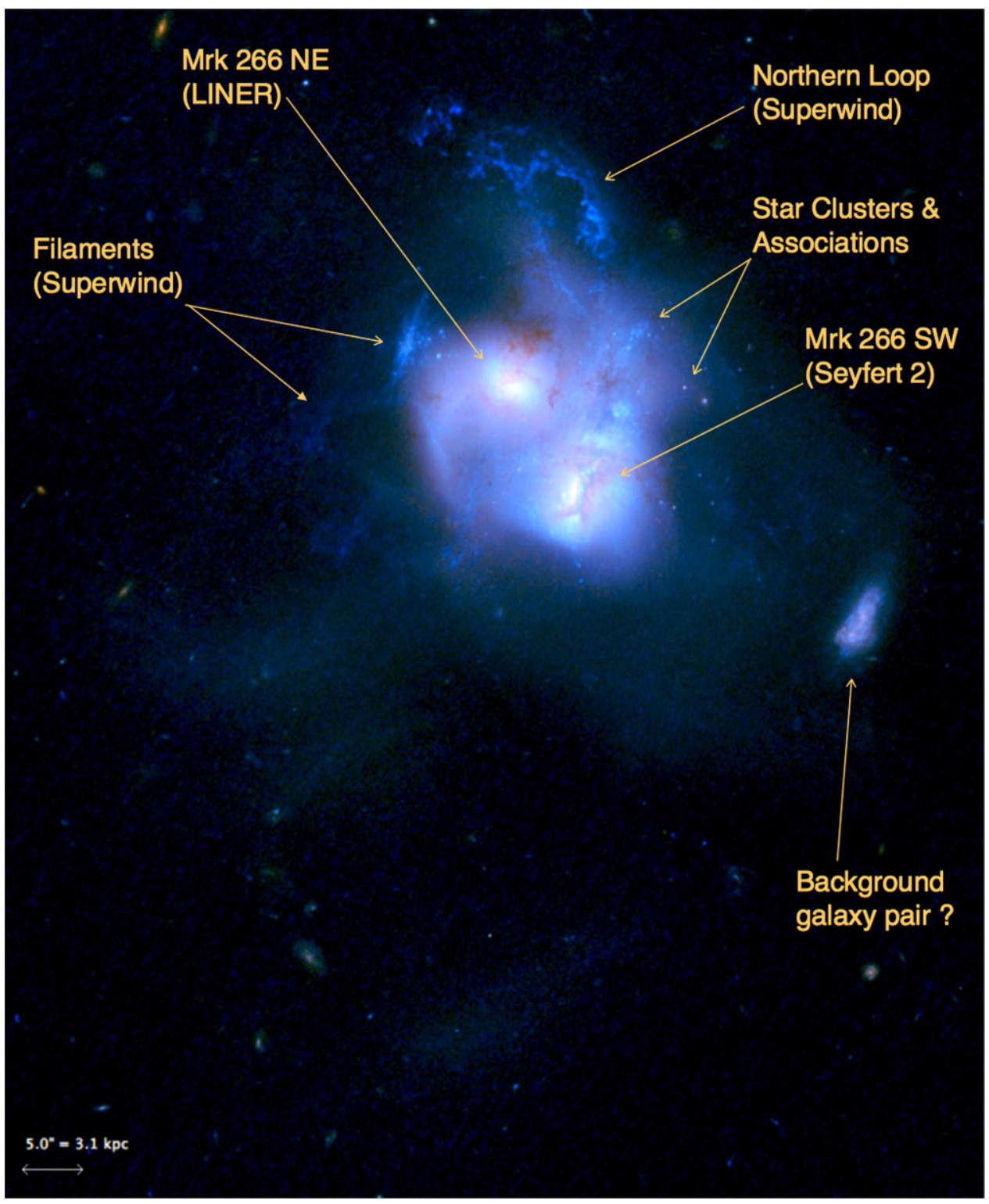

Figure 1. HST ACS $I$ band and $B$ band images combined to form a color composite image of the Mrk 266 system and its tidal features. North is up and east is left. The image field of view is $1^{\prime} .4 \times 1^{\prime} \cdot 6$, with a scale bar in the lower left corner. The color table has been chosen to maximize contrast for faint extended structures. The limiting surface brightness is 25.0 and $24.5 \mathrm{mag} \operatorname{arcsec}^{-2}$ in the $B$ and $I$ band, respectively. Labels identify major structures that are studied throughout this article.

(A color version of this figure is available in the online journal.)

X-ray properties alongside observations at other wavelengths, the data were taken from the Chandra Data Archive ${ }^{20}$ (ObSID: 2044) and re-processed using standard procedures in CIAO 4.0. The data were corrected for detector response and converted to flux density $\left(f_{v}\right)$ units. Details of the data reduction procedures can be found in Iwasawa et al. (2011). Figure 3 shows the resulting full band $(0.4-7 \mathrm{keV})$ image, the hard band $(2-7 \mathrm{keV})$ image, a smoothed version of the full band data, and the $20 \mathrm{~cm}$ radio continuum image from Mazzarella et al. (1988). Figure 4 illustrates apertures with parameters listed in Table 1 that were used to define regions of interest, and Figure 5 shows the X-ray spectra constructed in these regions.

\subsubsection{XMM-Newton Observations}

X-ray observations with XMM-Newton, reaching significantly higher energies $(10 \mathrm{keV})$ than Chandra $(7 \mathrm{keV})$, were acquired with the European Imaging Photon Camera (EPIC) on 2002 May 15 (PI: A. Read). The data obtained with the EPIC pn-CCD camera were utilized, providing an angular resolution of $\sim 6^{\prime \prime}$ FWHM and a spectral resolution of $(E / \delta E) \sim 40$. The observation was performed with the Thin Filter in the extended full-window imaging mode. The data set (0055990501) was taken from the XMM-Newton Science Archive ${ }^{21}$ and processed with standard procedures in the software packages SAS 8.1 and HEASoft 6.6 utilizing the latest calibration files (as of 2009 January). Time intervals with high particle background levels were discarded, leaving useful data with an effective exposure time of $13.2 \mathrm{ks}$. The count rate in the $0.4-10 \mathrm{keV}$ band is 0.19 count $\mathrm{s}^{-1}$. The X-ray emission is only marginally resolved by $X M M$, therefore the spectrum for the total system emission within a large aperture of $45^{\prime \prime}$ radius is produced here. Figure 6 shows the resulting $X M M$ spectrum of the integrated

20 http://cxc.harvard.edu/cda/

21 http://xmm.esac.esa.int/xsa/ 


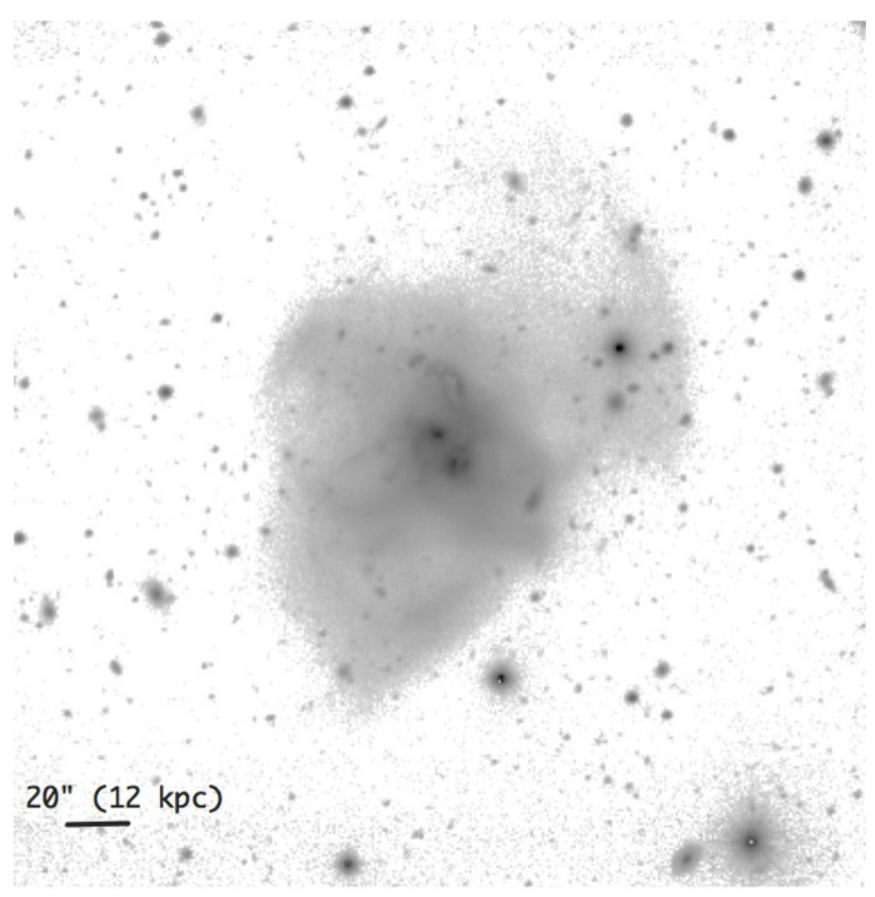

Figure 2. Result of stacking images in the $B, V$, and $I$ bands obtained with the University of Hawaii $2.2 \mathrm{~m}$ telescope (effective seeing $\sim 1^{\prime \prime} .5$ FWHM). The field of view is $150 \mathrm{kpc} \times 150 \mathrm{kpc}(4.2 \times 4.2)$, and a scale bar is provided in the lower left. Faint, low surface brightness emission spanning $2.9(\approx 103 \mathrm{kpc})$ is detected.
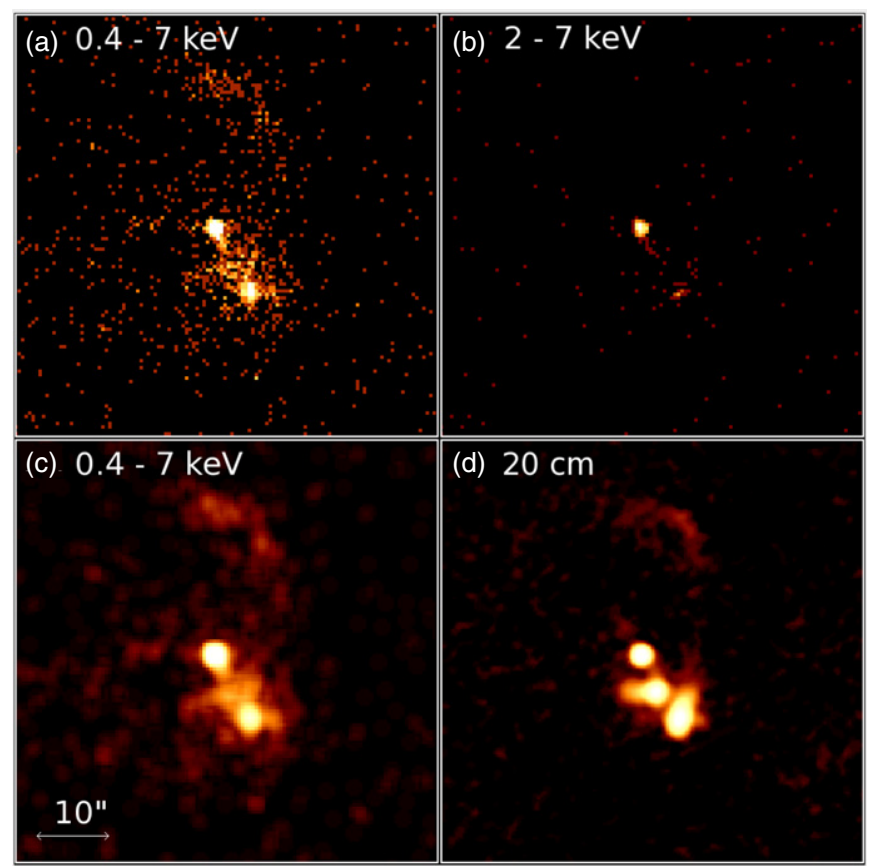

Figure 3. X-ray and radio continuum emission in Mrk 266: (a) Chandra 0.4-7 keV (full band) X-ray image; (b) 2-7 keV (hard band) X-ray image; (c) 0.4-7 keV X-ray data (same as panel (a)) smoothed with a 2 pixel Gaussian kernel; and (d) $20 \mathrm{~cm}$ radio continuum image (VLA) from Mazzarella et al. (1988). The scale bar indicates $10^{\prime \prime}$.

(A color version of this figure is available in the online journal.)

emission from the system. Key features include $\mathrm{Si}$ XIII $\mathrm{K} \alpha$ $(1.853 \mathrm{keV})$ emission, as also observed in the Chandra spectra of the two nuclei (Figure 5), and strong $\mathrm{Fe} \mathrm{K} \alpha$ line emission at $6.4 \mathrm{keV}$.

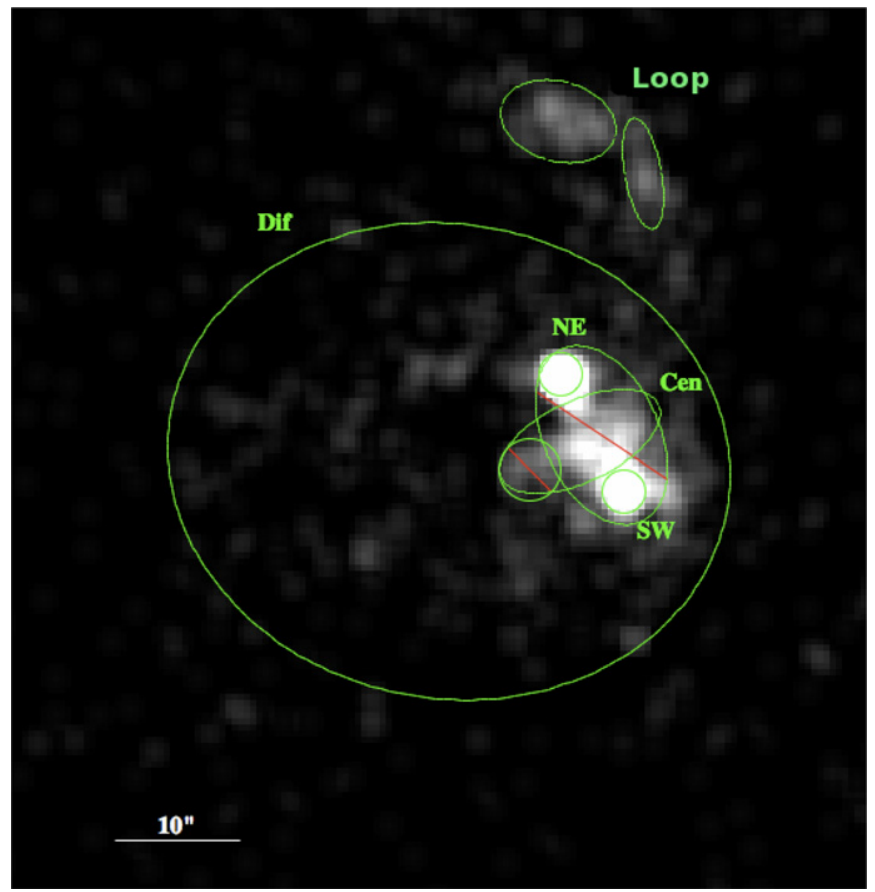

Figure 4. Elliptical apertures used to define regions of interest for extraction of X-ray spectra from the Chandra data: Mrk 266 NE ("NE"), Mrk 266 SW ("SW"), the central region between the nuclei ("Cen"), the Northern Loop ("Loop"), and surrounding diffuse emission ("Dif"). The two ellipses marked with red lines indicate regions subtracted from the ellipse labeled "Dif" to estimate the diffuse component. Parameters for the regions are listed in Table 1. A circular aperture of radius $33^{\prime \prime}$ used to measure the integrated system flux is not illustrated. (A color version of this figure is available in the online journal.)

Table 1

Elliptical Apertures for X-Ray Measurements

\begin{tabular}{lcccr}
\hline \hline Region & \multicolumn{1}{c}{$\begin{array}{c}\text { R.A., Decl. } \\
(\text { J2000) } \\
(1)\end{array}$} & $\begin{array}{c}a \\
\left({ }^{\prime \prime}\right)\end{array}$ & $b / a$ & $\begin{array}{r}\text { P.A. } \\
\left({ }^{\circ}\right)\end{array}$ \\
SW & $(13: 38: 17.31,+48: 16: 31.9)$ & 1.7 & 1.00 & $\ldots$ \\
NE & $(13: 38: 17.82,+48: 16: 41.2)$ & 1.7 & 1.00 & $\ldots$ \\
Center & $(13: 38: 17.66,+48: 16: 35.8)$ & 6.9 & 0.47 & 155 \\
Loop A & $(13: 38: 17.15,+48: 16: 57.2)$ & 4.5 & 0.32 & 10 \\
Loop B & $(13: 38: 17.83,+48: 17: 01.4)$ & 4.7 & 0.68 & 75 \\
Diffuse & $(13: 38: 18.71,+48: 16: 34.2)$ & 22.5 & 0.84 & 80 \\
Diffuse -1 & $-(13: 38: 17.49,+48: 16: 36.3)$ & 4.6 & 0.61 & 25 \\
Diffuse -2 & $-(13: 38: 18.07,+48: 16: 33.6)$ & 2.5 & 1.00 & $\ldots$ \\
Total & $(13: 38: 17.66,+48: 16: 35.8)$ & 33.0 & 1.00 & $\ldots$ \\
& & & &
\end{tabular}

Note. ${ }^{a}$ The diffuse emission is approximated by the flux within this large ellipse after subtraction of the flux in the two small ellipses in the following two rows, which are proceeded by a minus sign in this table and marked with red lines in Figure 4.

\subsubsection{X-Ray Measurements}

Spectral measurements and results from continuum model fitting of the Chandra and XMM-Newton observations are presented in Table 2. The sum of the emission in the elliptical regions misses some low surface brightness emission that is recovered in measurements of the total system in a circular aperture with a radius of $33^{\prime \prime}$. The total system fluxes measured by Chandra are, within the uncertainties (i.e., $20 \%$ in $F_{x}$ (soft) and $30 \%$ in $F_{x}$ (hard)), in agreement with the $X M M$ total flux measurements listed in Table 2 . There may be some contribution of photoionized gas (by the AGNs) in the soft X-ray emission, but the signal-to-noise ratio $(\mathrm{S} / \mathrm{N})$ is insufficient to fit this 

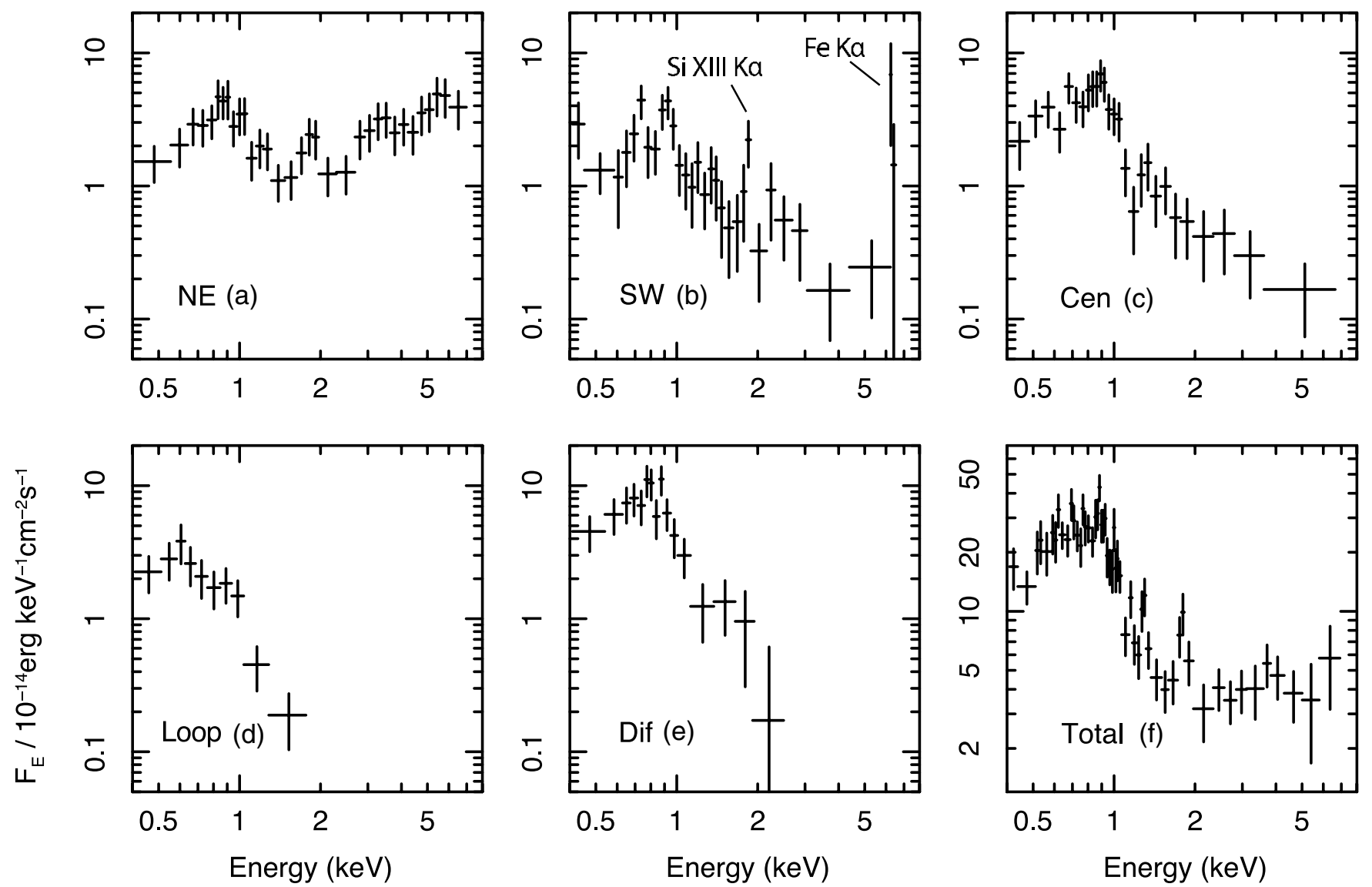

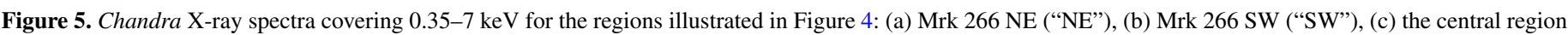

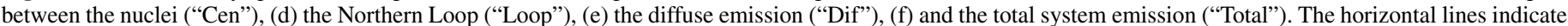
the variable-width energy bands, and the vertical lines are $1 \sigma$ error bars.

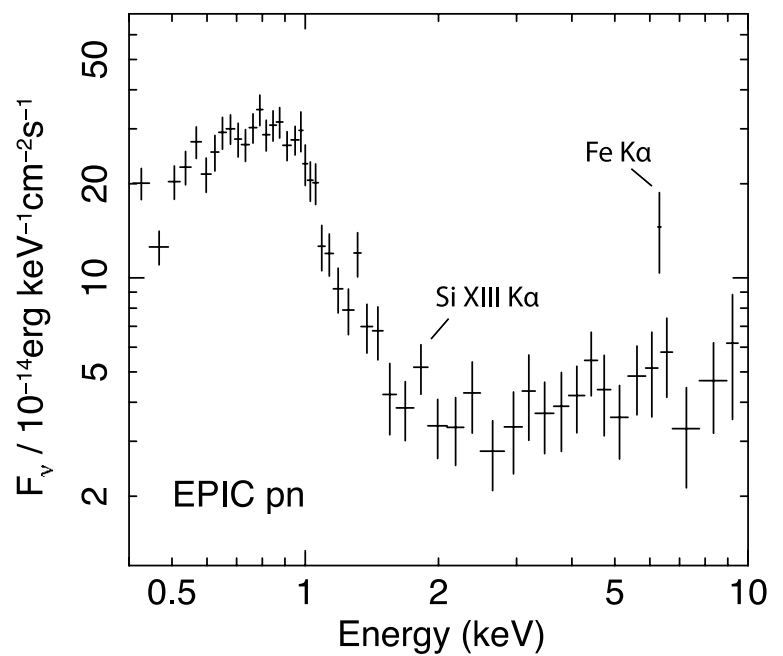

Figure 6. 0.5-10 keV X-ray spectrum of Mrk 266 (integrated system) measured with the EPIC instrument on board XMM-Newton. Primary features include the $\mathrm{Fe} \mathrm{K} \alpha$ emission line at $6.4 \mathrm{keV}$ and an $\mathrm{Fe} \mathrm{K}$ absorption edge at $7 \mathrm{keV}$.

component; therefore, the temperatures given in Table 2 are obtained from a model fit consisting of thermal emission due to star formation. Mrk $266 \mathrm{NE}$ has an AGN component, which is modeled by an absorbed power law with photon index 1.8 (fixed) and $N_{\mathrm{H}}=8.2_{-1.3}^{+1.8} \times 10^{22} \mathrm{~cm}^{-2}$; correcting for this absorption suggests an intrinsic hard-band flux of $2.7 \times 10^{-13} \mathrm{erg} \mathrm{cm}^{-2} \mathrm{~s}^{-1}$. There is a tail of hard X-ray emission in the source between the
Table 2

X-Ray Measurements

\begin{tabular}{lcccc}
\hline \hline Region & $F(\mathrm{Fe} \mathrm{K} \alpha)$ & $k T$ (soft) & $F_{x}$ (soft) & $F_{x}$ (hard) \\
& $(2)$ & $(3)$ & $(4)$ & $(5)$ \\
\hline Mrk 266 SW & $1.2_{-0.5}^{+0.7}$ & $0.77_{-0.08}^{+0.07}$ & 2.2 & 2.2 \\
Mrk 266 NE & $<1.6$ & $0.72_{-0.10}^{+0.11}$ & 3.0 & 14 \\
Between nuclei & $\ldots$ & $0.66_{-0.07}^{+0.06}$ & 2.6 & 1.0 \\
Northern Loop & $\ldots$ & $0.35_{-0.05}^{+0.05}$ & 1.4 & $\ldots$ \\
Diffuse Region & $\ldots$ & $0.54_{-0.04}^{+0.04}$ & 4.1 & $\ldots$ \\
Total $($ Chandra $)$ & $\ldots$ & $\ldots$ & 18 & 20 \\
Total $($ XMM) & $1.5_{-0.6}^{+0.8}$ & $\ldots$ & 18 & 23 \\
\hline
\end{tabular}

Notes. Measurements of the Chandra spectra in regions of interest; they are not corrected for absorption. Column 1: region of interest, as illustrated in Figure 4. Column 2: flux of the Fe K $\alpha$ line in units of $10^{-6}$ photons $\mathrm{cm}^{-2} \mathrm{~s}^{-1}$. The rest-frame energy measured from the $X M M$ spectrum for this feature is $6.50_{-0.05}^{+0.10} \mathrm{keV}$. The value for the NE nucleus is a $2 \sigma$ upper limit. Column 3 : temperature ( $k T$ in units of $\mathrm{keV})$ of the soft $(0.5-2 \mathrm{keV}) \mathrm{X}$-ray emission with $1 \sigma$ uncertainties. Column 4: flux in the soft $(0.5-2 \mathrm{keV}) \mathrm{X}$-ray band in units of $10^{-14} \mathrm{erg} \mathrm{cm}^{-2} \mathrm{~s}^{-1}$; the estimated relative uncertainty is $20 \%$. Column 5: flux in the hard (2-7 keV) X-ray band in units of $10^{-14} \mathrm{erg} \mathrm{cm}^{-2} \mathrm{~s}^{-1}$; the estimated relative uncertainty is $30 \%$. No flux is detected above $2 \mathrm{keV}$ in the Northern Loop or in the Diffuse Region. Mrk $266 \mathrm{SW}$ shows some emission above $2 \mathrm{keV}$, but most of the detected flux appears to originate in the Fe $\mathrm{K} \alpha$ line. The NE and SW galaxies also both show strong Si XIII emission at $1.8-1.9 \mathrm{keV}$.

nuclei, but its spectral shape is not well constrained; the $2-7 \mathrm{keV}$ flux given here is mostly due to this hard tail. 


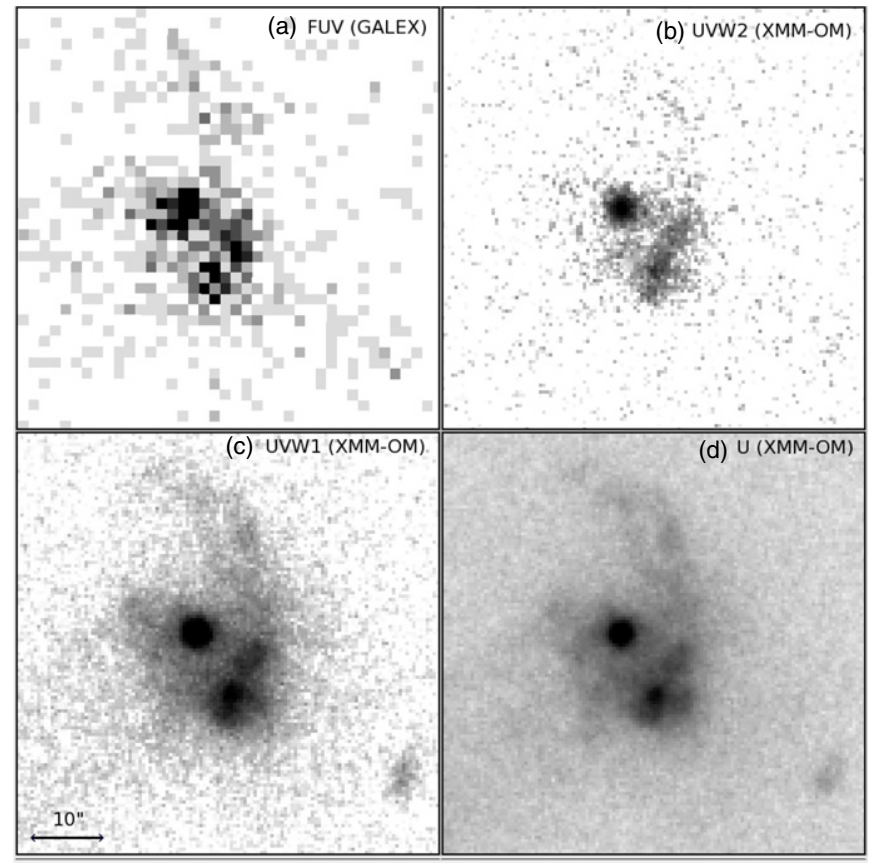

Figure 7. UV imagery of Mrk 266. (a) FUV (1528 $\AA$ ) band of GALEX,

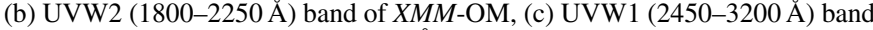
of $X M M-\mathrm{OM}$, and (d) U (3000-3900 Å) band of XMM-OM.

\subsection{Ultraviolet Imaging with GALEX and XMM-Newton OM}

Ultraviolet images of Mrk 266 were obtained on 2004 June 4 with an integration time of $111 \mathrm{~s}$ in the NUV (2271 $\mathrm{A})$ and FUV (1528 $\AA$ ) bands during the All Sky Imaging Survey (AIS) conducted by GALEX. The data were extracted from MAST (tile AISCHV3_104_01135) as provided by the standard GALEX data processing pipeline, and photometry was performed directly from these images using procedures outlined by Howell et al. (2010).

Contemporaneously with the X-ray observations by XMMNewton summarized above, the Optical/UV Monitor Telescope $(X M M-\mathrm{OM})$ obtained observations of Mrk 266 in the U (3000-3900 ̊), UVW1 (2450-3200 $)$, and UVW2 (1800-2250 A) filters with exposure times of 800,1000 , and $1400 \mathrm{~s}$, respectively. The $X M M-\mathrm{OM}$ instrument and data characteristics are described by Mason et al. (2001). These images have significantly better spatial resolution ( 2" FWHM) and higher sensitivity than the AIS observations from GALEX, and they also considerably extend the UV coverage. The reduced and calibrated $X M M-O M$ images, as produced using the methods documented by Kuntz et al. (2008), were obtained from MAST (data set 0055990501). Photometric measurements (presented in Section 2.6.2) were calibrated using zero points in Table 19 of the XMM-Newton Users Handbook, ${ }^{22}$ which are $19.1890,18.5662$, and 16.5719 AB mag in the U, UVW1, and UVW2 filters, respectively. Figure 7 displays the UV imagery of Mrk 266 in the FUV band of GALEX and in three filters of XMM-OM.

\subsection{Mid- and Far-infrared Imaging with Spitzer}

Imaging observations of Mrk 266 were obtained with the Spitzer Space Telescope as part of the Cycle 1 General Observing program for GOALS (PID 3672; PI: J. Mazzarella). The

\footnotetext{
22 http://heasarc.gsfc.nasa.gov/docs/xmm/uhb/
}

Infrared Array Camera (IRAC; Fazio et al. 2004) on board Spitzer was used to image the source at 3.6, 4.5, 5.8, and $8.0 \mu \mathrm{m}$ (AOR 12305408). Due to the presence of bright galactic nuclei, the high dynamic range (HDR) mode was utilized in all four bands. The HDR frames were composed of 1-2 s exposures to calibrate pixels that may have saturated in the $30 \mathrm{~s}$ exposures. The IRAC observations involved dithering to achieve redundancy to correct for cosmic-ray hits and bad pixels. The Multiband Imaging Photometer for Spitzer (MIPS; Rieke et al. 2004) was utilized to image Mrk 266 at 24, 70, and $160 \mu \mathrm{m}$ using the Photometry and Super Resolution mode (AOR 12349184). Each observation consisted of multiple $3 \mathrm{~s}$ integrations to insure adequate redundancy. Mapping cycles were used at each wavelength to correct the data for transients in the detectors. Details of the post-pipeline data processing and calibration of these data are given in J. M. Mazzarella et al. (2012, in preparation). Various Spitzer images and photometric measurements for Mrk 266 are incorporated into the following sections in conjunction with the analyses of data in other spectral regions.

\subsection{Mrk 266 from Radio Through X-Rays}

\subsubsection{Multi-waveband Imagery}

Figure 8 shows the structure of Mrk 266 in nine spectral regions spanning radio through X-rays using the observations discussed above. All images are displayed with the same orientation and scale to facilitate comparison. In each panel, contours of the $6 \mathrm{~cm}\left(4.885 \mathrm{GHz}, 0^{\prime \prime} .3 \times 0.0^{\prime} .4\right.$ beam $)$ image from Mazzarella et al. (1988) are superposed, primarily for astrometric reference. It is noteworthy that the elongated radio continuum structure with double peaks observed between the galaxy nuclei, as well as the elongated morphology of the SW galaxy, was confirmed in more sensitive $8.4 \mathrm{GHz}$ observations with $0^{\prime \prime} .27 \times 0^{\prime \prime} .22$ resolution (Leipski et al. 2006).

\subsubsection{Photometry and Spectral Energy Distributions}

Using the data displayed in Figure 8 and other passbands discussed above, aperture photometry was performed in elliptical regions parameterized in the Appendix (Table 11 and Figure 31). However, an alternative measurement procedure was necessary for the lower resolution far-infrared data. The spatial resolution of the MIPS images determined by the size of the Spitzer point-spread function (PSF) $(\lambda / D)$ are $6^{\prime \prime}, 18^{\prime \prime}$, and 40" FWHM at 24,70 , and $160 \mu \mathrm{m}$, respectively. The $24 \mu \mathrm{m}$ IRAC image (Figure 8(c)) clearly resolves the two galaxies, and it also reveals dust emission from the Northern Loop which is analyzed in Section 3.4.3. The $10^{\prime \prime}$ angular separation of the two galaxies is too small to be resolved by MIPS at 70 and $160 \mu \mathrm{m}$. However, as shown in Figure 8(b), the centroid of the $70 \mu \mathrm{m}$ emission clearly indicates that the SW galaxy dominates the far-infrared emission from the system. The centroid of the $160 \mu \mathrm{m}$ emission is also consistent with the SW galaxy providing the majority of the flux.

The 70 and $160 \mu \mathrm{m}$ flux densities for the two galaxies were estimated as follows. The relation derived by Xu et al. (2010),

$$
\begin{aligned}
\log \left(L_{\mathrm{ir}}\right)= & \log \left(L_{24}\right)+0.87( \pm 0.03) \\
& +0.56( \pm 0.09) \times \log \left(L_{8} / L_{24}\right)
\end{aligned}
$$

was used with measured monochromatic luminosities at 8 and $24 \mu \mathrm{m}$ to derive $L_{\mathrm{ir}}(1-1000 \mu \mathrm{m})$ estimates of $2.3 \times 10^{11}$, $6.6 \times 10^{10}$, and $3.4 \times 10^{11} L_{\odot}$ for Mrk $266 \mathrm{SW}, \mathrm{NE}$, and 


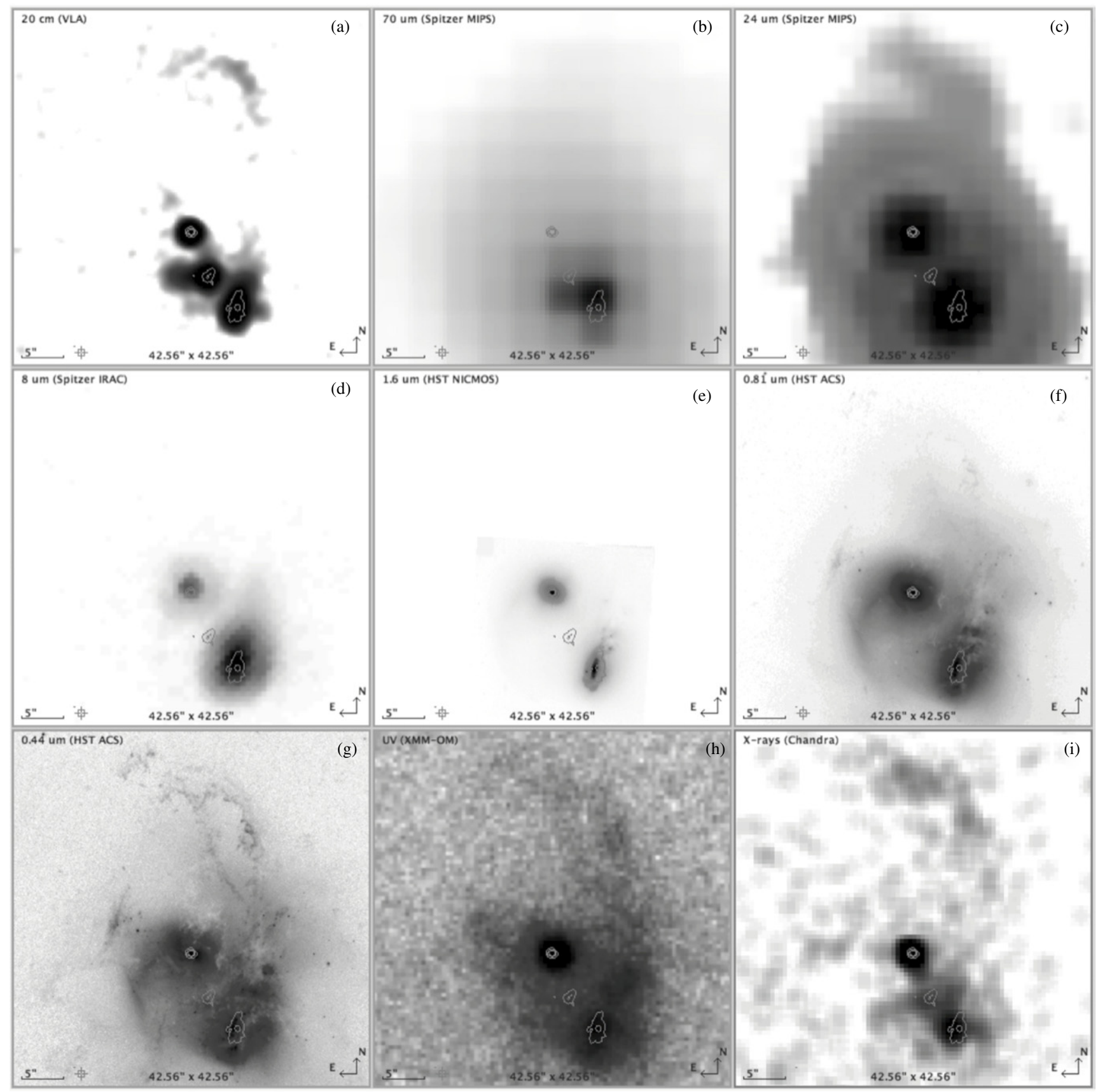

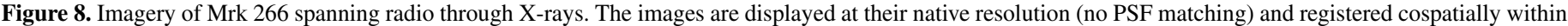

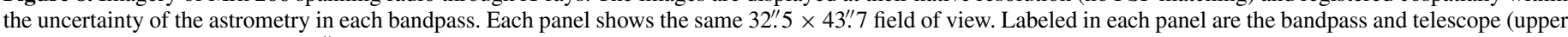

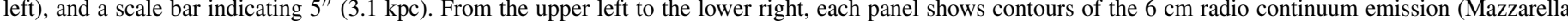

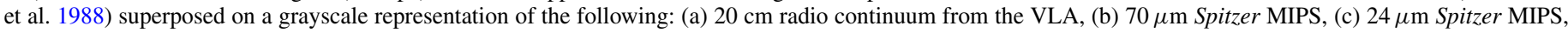

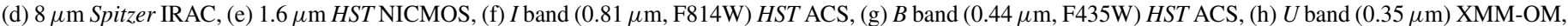
(i) X-rays (0.4-2 keV) Chandra.

the total system, respectively. Then, the ratios of $L_{70} / L_{\mathrm{ir}}=$ 0.58 and $L_{160} / L_{\mathrm{ir}}=0.19$ measured for the total system were applied to estimate the 70 and $160 \mu \mathrm{m}$ flux densities for the two galaxies (Table 13). The estimated uncertainty in the fluxes derived using this procedure is $\approx 20 \%$. Comparison of the summation of the estimated component flux densities with the measured total 70 and $160 \mu \mathrm{m}$ flux densities for the total system (Table 12) indicates consistency within the uncertainties.

Tables 12 and 13 (Appendix A.1) present photometry for the total system and for the components (SW, NE, the central region between the nuclei, and the Northern Loop). Figure 9 displays the resulting SEDs.

\subsection{Mid-infrared Spectroscopy with Spitzer}

Mid-infrared spectroscopy of Mrk 266 obtained in staring mode with the Infrared Spectrograph (IRS; Houck et al. 2004) on board Spitzer have been published by Brandl et al. (2006), Dudik et al. (2007), and Bernard-Salas et al. (2009). Unfortunately, these observations suffer from aperture effects that resulted in missing emission from some components in different spectral regions. Appendix A. 2 provides clarification of which emission features have been measured for each component of Mrk 266.

In order to overcome the limited spatial extent of the staringmode observations, the IRS spectral map obtained on 2005 


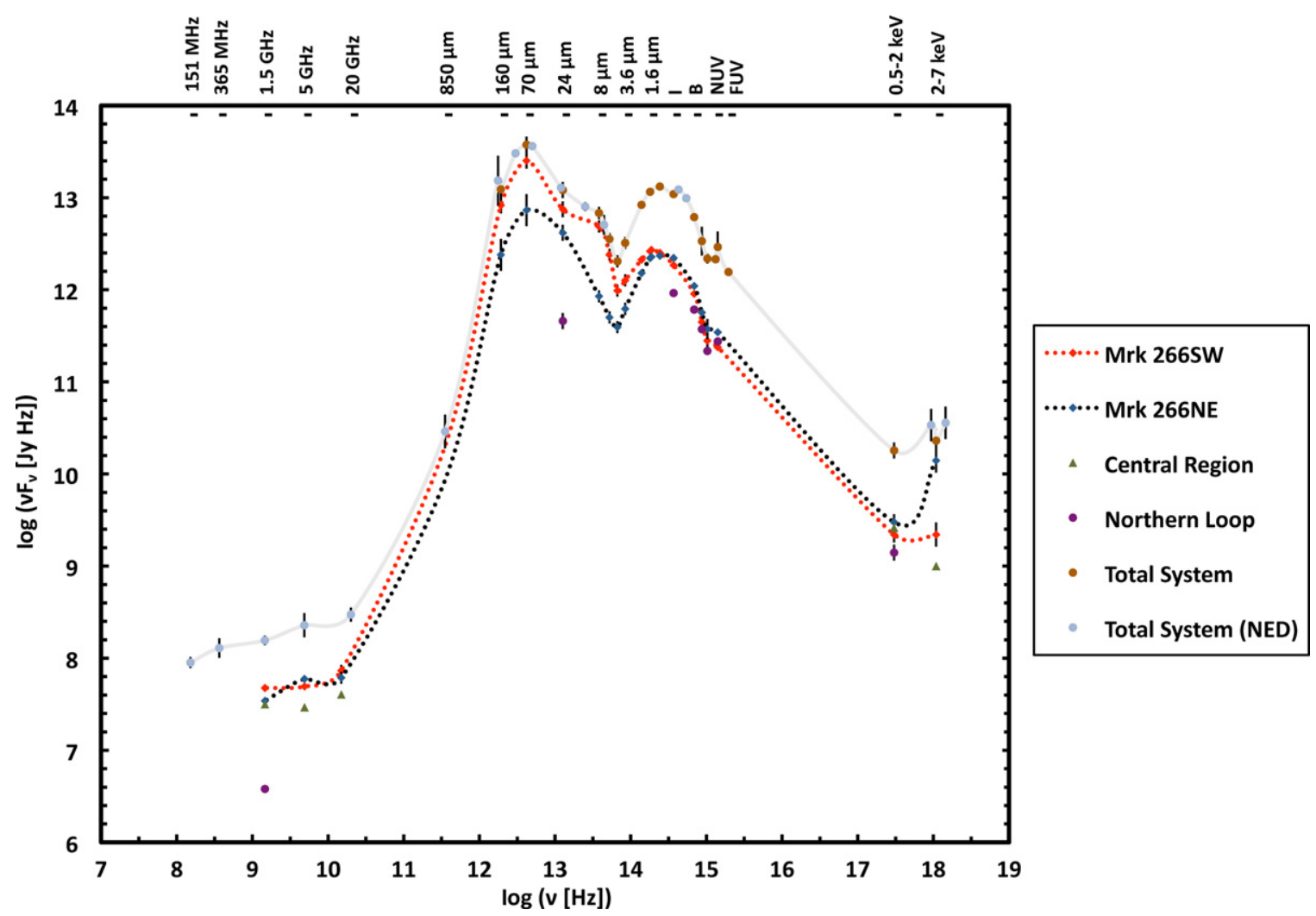

Figure 9. SEDs for Mrk 266 SW (red), Mrk 266 NE (blue), the Central Region (green) between the nuclei, the Northern Loop (purple), and the integrated emission from the Total System (brown). The legend shows the color-coding used to identify the various components. Most of the photometric measurements are presented here for the first time and some data were selected from NED, as listed in Tables 12 and 13 . The monochromatic fluxes plotted are computed via $\log \left(v f_{v}[\mathrm{Jy} H z]\right)$.

(A color version of this figure is available in the online journal.)

January 10 available in the Spitzer archive (program P03269; PI: J. Gallimore; AOR 12459264) was used to construct spectra for each nucleus and for the total system. Spectral mapping with the SL module consisted of 13 integrations, each $6 \mathrm{~s}$ in duration and stepped perpendicular to the slit with an interval of 1 1.8 (half the slit width). Mapping with the LL module was performed with five integrations, each $6 \mathrm{~s}$ in duration and stepped perpendicular to the slit with an interval of 4.85 (half the slit width). The individual calibrated spectra produced by version S15.3 of the Spitzer Science Center pipeline were used to construct a spectral cube with the CUBISM package (Smith et al. 2007). In all cases, background observations were assembled from the non-primary slit and subtracted from the data. CUBISM was used to construct images over specific wavelength ranges and spectral features, including 5.5, 10, and $14 \mu \mathrm{m}$ continuum and continuum-subtracted $6.2,7.7$, and 11.3 $\mu \mathrm{m}$ PAH, $9.7 \mu \mathrm{m} \mathrm{H}_{2} \mathrm{~S}(3)$, and [S IV] $10.5 \mu \mathrm{m}$ emission lines. These images are presented in Figure 10, where the first panel illustrates rectangular apertures used in CUBISM to extract one-dimensional (1D) spectra.

With a pixel size of $5^{\prime \prime} .1 \times 5^{\prime \prime} .1$ and a spatial resolution of $10^{\prime \prime}$ on the red end, we cannot use the LL observations $(14-38 \mu \mathrm{m})$ to separate the two galaxies as we can with the SL data $(5.2-14.5 \mu \mathrm{m})$. Further, the LL map did not completely cover the NE nucleus. A "total" SL+LL 1D spectrum was extracted that contains most of the integrated emission of the system over $5-38 \mu \mathrm{m}$ in a region $4 \times 4 \mathrm{LL}$ pixels in size (outlined in brown in Figure 10(a)). Another 1D spectrum was extracted that fully covers both galaxies in the 5-14 $\mu \mathrm{m}$ SL spectrum in a region $13 \times 13$ SL pixels in size, where each pixel is $1^{\prime \prime} .8 \times 1^{\prime \prime} .8$. The resulting $1 \mathrm{D}$ spectra, representing the sum of the emission from both galaxies, are presented in Figure 11 (SL + LL) and in Figure 12(a) (SL only), respectively. In addition, 1D extractions from the SL map were constructed that provide a clean separation between the NE and SW nuclei; these regions are shown as red and blue rectangles (each covering $5 \times 5$ SL pixels) in Figure 10(a), and the resulting 1D spectra are presented in Figures 12(b) and (c). Due to the very high mid-infrared surface brightness of the galaxies, with a spatial resolution of 3".6 (two 1".8 pixels), even the SL spectral map does not enable a reliable separation of the nuclear emission from the diffuse emission surrounding the nuclei.

This is the first time that mid-infrared spectra are available for each nucleus in Mrk 266 separately, as well as the integrated emission from both galaxies. Table 3 lists measured line fluxes and equivalent widths for the total system and, where possible, the individual galaxies. The fluxes were converted from surface brightness units $\left(\mathrm{W} \mathrm{m} \mathrm{m}^{-2} \mathrm{sr}^{-1}\right.$ ) to $\mathrm{W} \mathrm{cm}^{-2}$ by multiplying by the extraction aperture. Most of the spectral features in the SL + LL spectrum of the integrated emission from the galaxy pair (Figure 11) were measured using the PAHFIT package (Smith et al. 2007), which includes decomposition of various spectral components and an extinction correction based on a fit with a $\tau_{9.7 \mu \mathrm{m}}=0.30$ dust screen, corresponding to $A_{V} \approx 5.7 \mathrm{mag}$. However, since the nuclei were resolved only in the SL data (Figures 12(b) and (c)), and PAHFIT produces the most reliable continuum and feature fits when SL and LL are combined, we have used a spline-fitting technique (Hony et al. 2001; Peeters et al. 2002) to measure the SL features listed in Table 3 for the SW and NE galaxies. This further allows for 

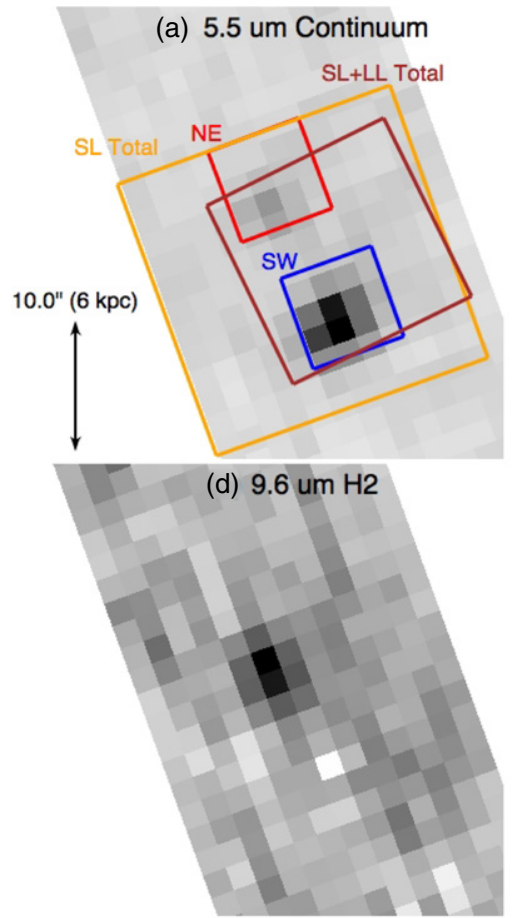

(g) 11.3 um PAH (b) 6.2 um PAH

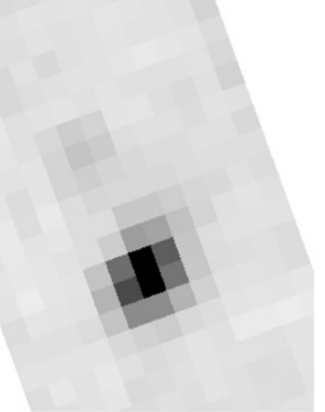

(e) 10 um Continuum

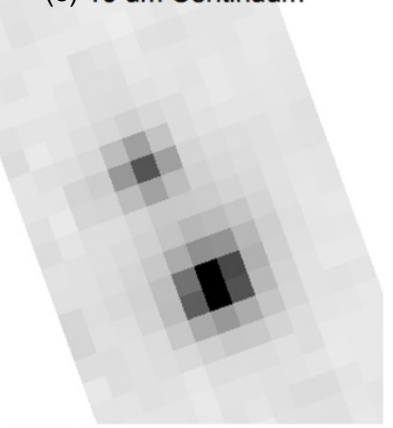

(h) 14 um Continuum (c) 7.7 um PAH
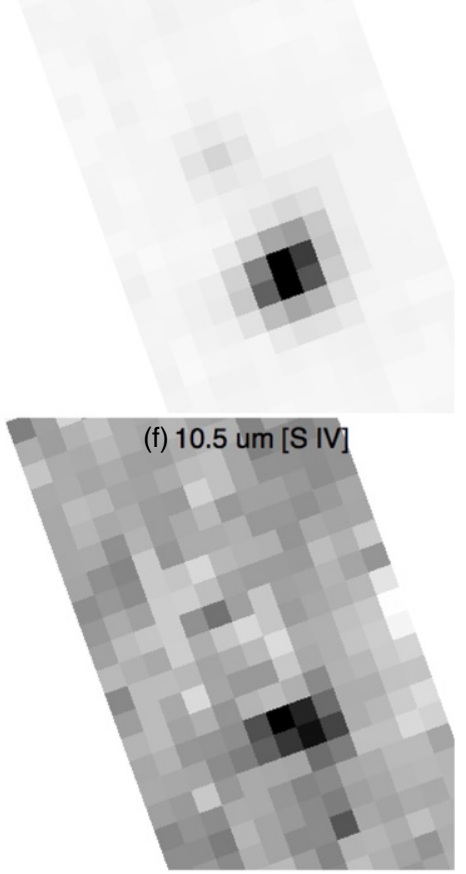
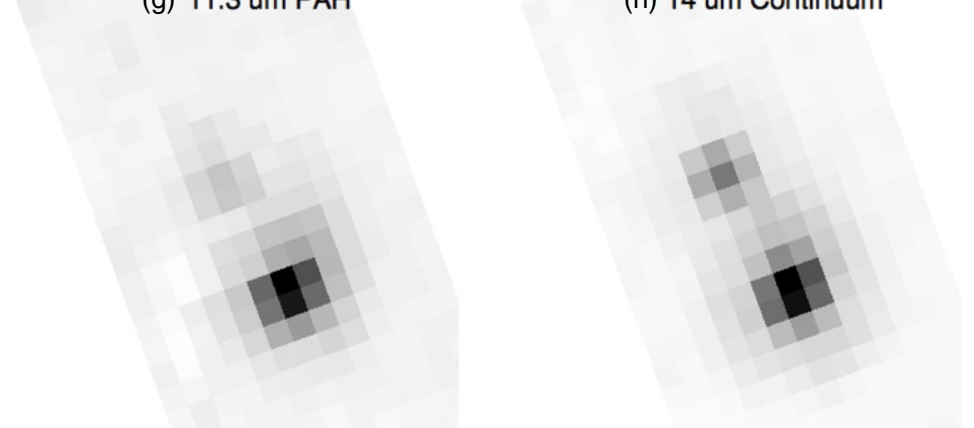

Figure 10. Image slices through the 2D spectral IRS map. Panels (a)-(h) show images in the indicated spectral features (pure line or continuum emission). All images have north up and east left, and have the same scale indicated by the bar in panel (a). The colored rectangles overlaid on the $5.5 \mu \mathrm{m}$ image in panel (a) indicate the regions of the 2D spectral cube used to extract 1D spectra. Brown box: "total" emission in the SL+LL modules plotted in Figure 11 (Mrk 266NE was not fully covered by the LL aperture); orange box: "total" emission in the SL module plotted in Figure 12(a); red box: emission from the NE nucleus in the SL module plotted in Figure 12(b); blue box: emission from the SW nucleus in the SL module plotted in Figure 12(c).

(A color version of this figure is available in the online journal.)

direct comparison to other sources in the literature where this technique has been employed. Spline fitting was also utilized for the polycyclic aromatic hydrocarbon (PAH) equivalent width (EQW) measurements to estimate the local continuum and the silicate absorption strength. As noted by Smith et al. (2007), spline fitting systematically underestimates PAH fluxes compared to full spectral decomposition via PAHFIT. The various measurements are identified in Table 3 with superscripts $p$ (PAHFIT) and $s$ (spline fitting), respectively.

\section{ANALYSIS AND DISCUSSION}

Mrk 266 is a complex collection of many different physical subsystems, each of which are identified in Figure 1 and discussed in the following subsections. At the macro level, Mrk 266 is clearly a merging system with two distinct AGNs coupled with strong spatial differences in current star formation, outflow, merger dynamics, and AGN heating. But within that framework there are several specific questions that we can directly address using our multiwavelength data set: What are the structural parameters and underlying stellar populations of the two host galaxies? Is there evidence for dynamic outflow from each nucleus? Is the NE LINER a genuine AGN or is it powered by star formation? Is the origin of the observed diffuse emission tidal or due to galactic superwinds? What are the properties of the suspected star clusters (SCs) that are forming in the merging system? Is Mrk 266 a unique dual AGN system or are we observing a short-lived phase of an evolutionary process that commonly occurs when two massive, dusty disks merge?

\subsection{Interacting/Merging Galaxies Revealed}

\subsubsection{Morphology and Bulge/Disk/Bar Decomposition}

High-resolution HST imagery (Figure 1) has revealed a wealth of morphological structures within Mrk 266. At optical wavelengths $(B$ and $I)$ there are two distinct galaxies within a diffuse and highly asymmetric envelope. Patchy dust obscuration and clumpy star formation regions are evident on a variety of scales. The underlying structure of the two galaxies is best revealed 


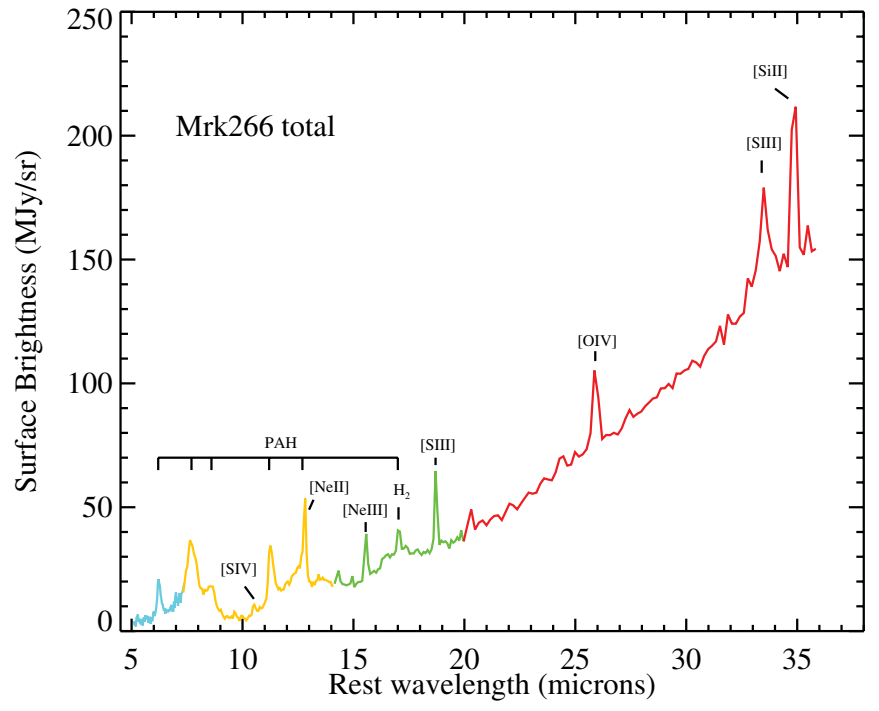

Figure 11. 1D spectrum of Mrk 266 extracted from a low-resolution spectral map constructed from the SL and LL modules of the IRS. The region used to produce this spectrum is shown as a brown box in Figure 10. This is our best attempt to represent the integrated emission for both galaxies across the full $5-38 \mu \mathrm{m}$ range covered by the low-resolution modules of the IRS, with the caveats noted in the text. The colors in this spectrum represent the various orders in the IRS instrument and are not related to the colors in Figure 10.

(A color version of this figure is available in the online journal.)

in the NICMOS $H$-band image. The SW galaxy (Figure 13) is a barred spiral with two arms and predominant dust features, and the estimated Hubble type is SBb (pec). The NE galaxy (Figure 14) has no discernible spiral arms and appears to be Hubble type $\mathrm{S} 0$ or $\mathrm{S} 0 / \mathrm{a}$ (pec).

In the extremities of the system are three notable features at optical wavelengths (Figure 1). (1) Extending 25" (15 kpc) to the north is a fragmented, filamentary feature known as the Northern Loop; this structure is examined in detail in Section 3.4. (2) Faint emission extending $\sim 60^{\prime \prime}(36 \mathrm{kpc}$ ) to the south and $\sim 40^{\prime \prime}(24 \mathrm{kpc})$ to the southeast from the center of the system appears to be tidal debris. The deep $B+V+I$ image (Figure 2) reveals much fainter, asymmetric emission spanning $\approx 103 \mathrm{kpc}(2 \cdot 9)$. The peculiar morphology and vast extent of this emission are consistent with numerical simulations of tidal debris created during a major merger (e.g., Mihos 1999; Cox et al. 2006). (3) Approximately 25" to the southwest are two objects that could be either knots near the end of a tidal tail or background galaxies. The latter interpretation is favored due to what appear to be compact nuclei and spiral arms. If these are background galaxies with a physical size and separation similar to Mrk 266 , their projected diameters $\left(\approx 3^{\prime \prime}\right)$ and separation $\left(\approx 2^{\prime \prime}\right)$ imply they are $\sim 5$ times further away than Mrk 266 (distance $\sim 650 \mathrm{Mpc}, z \sim 0.15$ ); redshift measurements are needed for confirmation.

Using GALFIT (Peng et al. 2002), quantitative isophotal analysis was performed on both component galaxies in the three $H S T$ bands. At $1.6 \mu \mathrm{m}$ ( $H$ band) where dust obscuration is minimal and thus permits the most reliable decomposition of the underlying galaxies, various trial fits involving possible point source, bulge, disk, and/or bar components were made; potential nuclei were modeled using a PSF generated from the Tiny Tim package (Krist 1993). ${ }^{23}$ Figure 15 shows the components of the best-fitting models in the $H$ band. Due to
Table 3

Measurements from Low-res Spectral Mapping

\begin{tabular}{|c|c|c|}
\hline Feature & $\begin{array}{c}\text { Flux } \\
\left(1 \times 10^{-20} \mathrm{~W} \mathrm{~cm}^{-2}\right) \\
(2)\end{array}$ & $\begin{array}{l}\text { EQW } \\
(\mu \mathrm{m}) \\
(3)\end{array}$ \\
\hline \multicolumn{3}{|c|}{ Mrk 266 Total $^{\mathrm{a}}$} \\
\hline $6.2 \mu \mathrm{m} \mathrm{PAH}$ & $40.2 \quad( \pm 1.8)^{\mathrm{b}}$ & $0.47( \pm 0.03)^{c}$ \\
\hline $\mathrm{H}_{2} \mathrm{~S}(5) 6.91 \mu \mathrm{m}$ & $0.66 \quad( \pm 0.50)^{\mathrm{b}}$ & $\ldots$ \\
\hline [Ar II] $6.99 \mu \mathrm{m}$ & $2.34( \pm 0.72)^{\mathrm{b}}$ & $\ldots$ \\
\hline $7.7 \mu \mathrm{m}$ PAH complex & $163.0 \quad( \pm 6.1)^{\mathrm{b}}$ & $0.46( \pm 0.02)^{\mathrm{c}}$ \\
\hline $8.3 \mu \mathrm{m}$ PAH & $13.7 \quad( \pm 1.8)^{\mathrm{b}}$ & $\ldots$ \\
\hline $8.6 \mu \mathrm{m}$ PAH & $26.8 \quad( \pm 1.5)^{\mathrm{b}}$ & $0.15( \pm 0.01)^{c}$ \\
\hline [Ar III] $9.02 \mu \mathrm{m}$ & $0.80 \quad( \pm 0.49)^{\mathrm{b}}$ & $\ldots$ \\
\hline $\mathrm{H}_{2} \mathrm{~S}(3) 9.65 \mu \mathrm{m}$ & $1.42 \quad( \pm 0.38)^{\mathrm{b}}$ & $\ldots$ \\
\hline$\left[\mathrm{S} \mathrm{IV}_{\mathrm{IV}} 10.51 \mu \mathrm{m}\right.$ & $1.59( \pm 0.29)^{\mathrm{b}}$ & $\ldots$ \\
\hline $11.3 \mu \mathrm{m}$ PAH complex & $31.9 \quad( \pm 1.2)^{\mathrm{b}}$ & $0.51( \pm 0.01)^{\mathrm{c}}$ \\
\hline $12.0 \mu \mathrm{m}$ РAH & $11.85( \pm 0.83)^{\mathrm{b}}$ & $\ldots$ \\
\hline $\mathrm{H}_{2} \mathrm{~S}(2) 12.27 \mu \mathrm{m}$ & $0.89 \quad( \pm 0.15)^{\mathrm{b}}$ & $\ldots$ \\
\hline $12.6 \mu \mathrm{m}$ PAH complex & $23.78( \pm 0.73)^{\mathrm{b}}$ & $\ldots$ \\
\hline$[\mathrm{Ne}$ II $] 12.81 \mu \mathrm{m}$ & $6.78( \pm 0.18)^{\mathrm{b}}$ & $\ldots$ \\
\hline $13.6 \mu \mathrm{m} \mathrm{PAH}$ & $8.86 \quad( \pm 0.70)^{b}$ & $\ldots$ \\
\hline $14.2 \mu \mathrm{m} \mathrm{PAH}$ & $2.80 \quad( \pm 0.52)^{\mathrm{b}}$ & $\ldots$ \\
\hline$[\mathrm{Ne} \mathrm{III}] 15.56 \mu \mathrm{m}$ & $3.57( \pm 0.24)^{\mathrm{b}}$ & $\ldots$ \\
\hline $16.4 \mu \mathrm{m}$ PAH & $2.08 \quad( \pm 0.38)^{\mathrm{b}}$ & $\ldots$ \\
\hline $\mathrm{H}_{2} \mathrm{~S}(1) 17.04 \mu \mathrm{m}$ & $1.61( \pm 0.19)^{\mathrm{b}}$ & $\ldots$ \\
\hline $17 \mu \mathrm{m}$ PAH complex & $19.4 \quad( \pm 1.7)^{\mathrm{b}}$ & $\ldots$ \\
\hline $17.4 \mu \mathrm{m} \mathrm{PAH}$ & $1.01 \quad( \pm 0.36)^{b}$ & $\ldots$ \\
\hline$[\mathrm{S} \mathrm{III]} 18.71 \mu \mathrm{m}$ & $4.16 \quad( \pm 0.28)^{\mathrm{b}}$ & $\ldots$ \\
\hline$[\mathrm{O}$ IV $] 25.9 \mu \mathrm{m}$ & $4.52 \quad( \pm 0.14)^{b}$ & $\ldots$ \\
\hline$[\mathrm{S} \mathrm{III]} 33.51 \mu \mathrm{m}$ & $3.71 \quad( \pm 0.28)^{\mathrm{b}}$ & $\ldots$ \\
\hline$[\mathrm{Si}$ II $] 34.86 \mu \mathrm{m}$ & $5.69 \quad( \pm 0.39)^{\mathrm{b}}$ & $\cdots$ \\
\hline \multicolumn{3}{|c|}{ Mrk $266 \mathrm{SW}^{\mathrm{d}}$} \\
\hline $6.2 \mu \mathrm{m}$ PAH & $17.78( \pm 0.35)^{\mathrm{c}}$ & $0.67( \pm 0.03)^{\mathrm{c}}$ \\
\hline $7.7 \mu \mathrm{m}$ PAH complex & $30.46 \quad( \pm 0.35)^{\mathrm{c}}$ & $0.54( \pm 0.01)^{c}$ \\
\hline $8.6 \mu \mathrm{m}$ PAH & $5.45 \quad( \pm 0.14)^{\mathrm{c}}$ & $0.17( \pm 0.01)^{\mathrm{c}}$ \\
\hline$[\mathrm{S} \mathrm{IV}] 10.51 \mu \mathrm{m}$ & $0.95( \pm 0.13)^{\mathrm{c}}$ & $\ldots$ \\
\hline $11.3 \mu \mathrm{m}$ PAH complex & $10.376( \pm 0.088)^{\mathrm{c}}$ & $0.73( \pm 0.01)^{\mathrm{c}}$ \\
\hline$[\mathrm{Ne} \mathrm{II}] 12.81 \mu \mathrm{m}$ & $6.15 \quad( \pm 0.99)^{\mathrm{c}}$ & $\cdots$ \\
\hline \multicolumn{3}{|c|}{ Mrk $266 \mathrm{NE}^{\mathrm{d}}$} \\
\hline $6.2 \mu \mathrm{m}$ PAH & $2.418( \pm 0.019)^{\mathrm{c}}$ & $0.4( \pm 0.1)^{\mathrm{c}}$ \\
\hline $7.7 \mu \mathrm{m}$ PAH complex & $2.86 \quad( \pm 0.28)^{\mathrm{c}}$ & $0.25( \pm 0.03)^{\mathrm{c}}$ \\
\hline $8.6 \mu \mathrm{m}$ PAH & $0.69 \quad( \pm 0.11)^{\mathrm{c}}$ & $0.10( \pm 0.02)^{c}$ \\
\hline $\mathrm{H}_{2} \mathrm{~S}(3) 9.65 \mu \mathrm{m}$ & $0.52( \pm 0.14)^{\mathrm{c}}$ & $\ldots$ \\
\hline $11.3 \mu \mathrm{m}$ PAH complex & $2.170( \pm 0.078)^{\mathrm{c}}$ & $0.34( \pm 0.02)^{\mathrm{c}}$ \\
\hline $\mathrm{H}_{2} \mathrm{~S}(2) 12.27 \mu \mathrm{m}$ & $0.308( \pm 0.063)^{\mathrm{c}}$ & $\ldots$ \\
\hline$[\mathrm{Ne} \mathrm{II}] 12.81 \mu \mathrm{m}$ & $1.84( \pm 0.29)^{\mathrm{c}}$ & $\ldots$ \\
\hline
\end{tabular}

Notes.

a Spectral measurements for the integrated emission from both galaxies in Mrk 266. Features in the 5.2-14.5 $\mu \mathrm{m}$ (SL) and 14.5-38 $\mu \mathrm{m}$ (LL) regions were measured from the $1 \mathrm{D}$ spectrum plotted in Figure 11; this region is 4 $\times 4$ LL pixels in size, where each pixel is $5^{\prime \prime} 1 \times 5^{\prime \prime}$. 1 , as outlined in brown in Figure 10(a).

b These line fluxes were measured using PAHFIT and include an extinction correction based on a fit with a $\tau_{9.7 \mu \mathrm{m}}=0.30$ dust screen, which corresponds to $A_{V} \approx 5.7 \mathrm{mag}$.

c EQW measurements for the PAH features were measured using a splinefitting technique for estimating the local continuum. Spline fitting was also used to estimate fluxes of features in the SL spectra of the individual galaxies which could not be measured with PAHFIT. See the text for details.

d Spectral features measured from the SL spectra of Mrk 266 SW and NE as plotted in Figures 12(c) and (b). These 1D spectra were extracted from the blue and red regions of the spectral map shown in Figure 10, which have dimensions of $5 \times 5$ SL pixels, where each pixel is $1^{\prime \prime} .8 \times 1^{\prime \prime} .8$.

$\overline{23}$ http://www.stsci.edu/software/tinytim/tinytim.html 


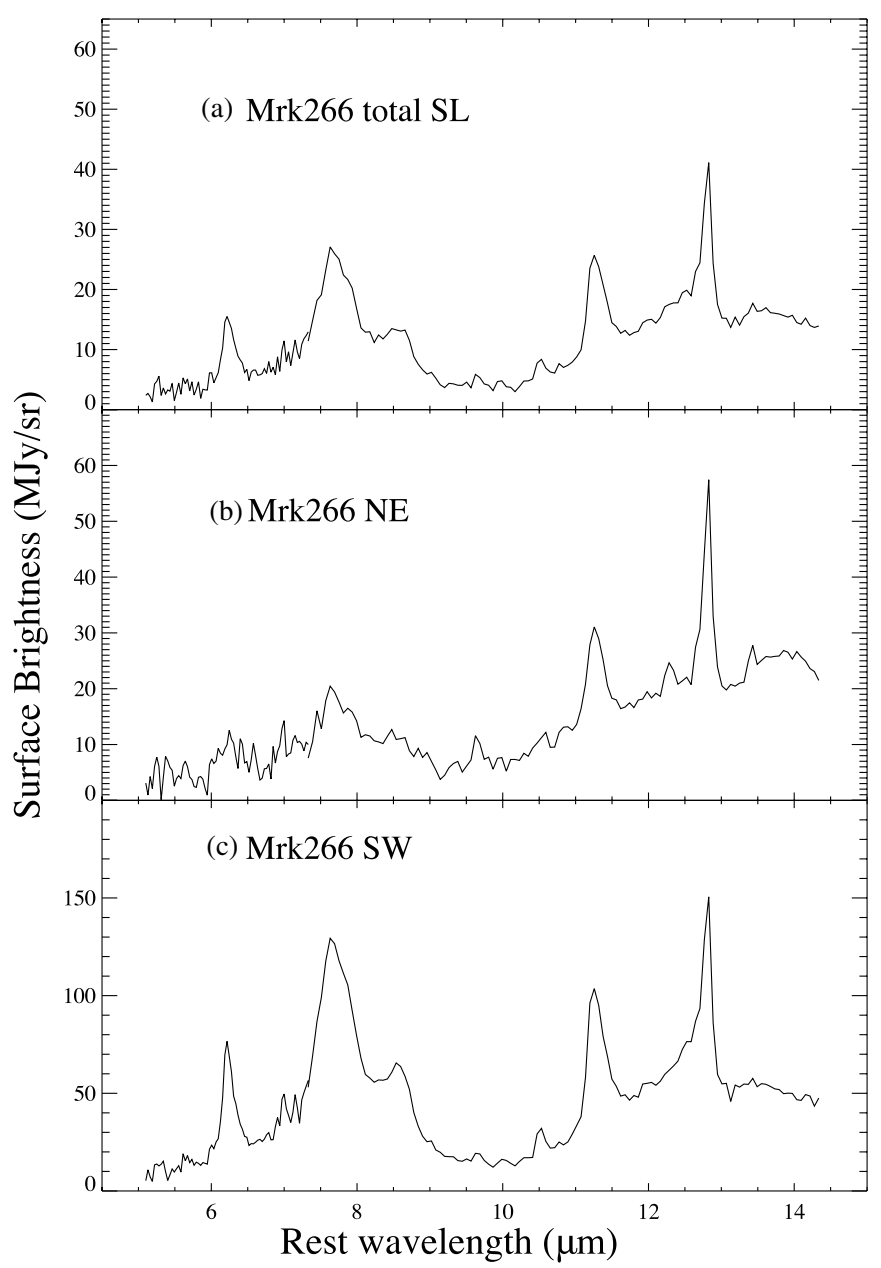

Figure 12. The spatial resolution of the SL observations permits a clean separation of the two galaxies that is not possible with the LL observations. Each panel is a 1D extraction from the SL spectral map presented in Figure 10: (a) orange region covering the entire region, (b) red region containing Mrk $266 \mathrm{NE}$, and (c) blue region containing Mrk 266 SW.

extensive, patchy dust obscuration and complex star-forming regions, only models with possible bulge and disk components were considered in the $B$ and $I$ bands. Subtraction of the bestfitting models from the direct images is useful to elucidate further fine structures, as shown visually for all three bands in Figure 16. The results of this structural analysis at $H$ band are the following. (1) The SW galaxy has no detected pointsource emission; its nucleus is therefore obscured by dust even at $1.6 \mu \mathrm{m}$. (2) The NE galaxy exhibits no significant disk component and therefore can be modeled using only a point source plus bulge profile. (3) Approximately $10 \%$ of the total $\mathrm{H}$-band light is in the form of diffuse emission which is likely tidal in origin (Figure 15(a)). In the optical bands the residual images reveal the following. (1) The SW galaxy has many knots of emission which are likely SCs or associations; these features are analyzed in detail in Section 3.6. (2) The largest features in the $B$-band residual image appear to be giant star-forming regions (superassociations) situated on both ends of the stellar bar. This phenomenon, in which superassociations occur on both sides of stellar bars much more frequently than they occur on a single side, was pointed out by Magtesian \& Khachikian (1991). (3) The NE galaxy has numerous radial, asymmetric filaments (most pronounced in the $B$ band) indicative of biconical outflow from the AGN; this region is analyzed more fully in Section 3.2.4. Parameters from the GALFIT modeling are listed in Table 4. Adopting a distance modulus of $35.55 \mathrm{mag}$ (see Section 1.3), the apparent $\mathrm{H}$ magnitudes (Vega system) of Mrk $266 \mathrm{SW}$ and NE (Table 4) correspond to remarkably similar absolute magnitudes of $M_{H}(\mathrm{SW})=-24.32$ and $M_{H}(\mathrm{NE})=-24.16$.

\subsubsection{Galaxy Luminosities and Derived Stellar Masses}

The $H$-band $(1.6 \mu \mathrm{m})$ luminosity function (LF) of galaxies in the Coma Cluster has been approximated as a Schechter function with $L_{H}^{*}=-23.9 \mathrm{mag}$ (de Propris et al. 1998). Therefore, Mrk $266 \mathrm{SW}$ and $\mathrm{NE}$ are $\approx 50 \%$ and $30 \%$ more luminous than the typical $L_{H}^{*}$ galaxy in the local universe. Applying the mean mass-to-light ratio at $H$ band of $4.6\left(M_{\odot} / L_{\odot}\right)$ for spiral galaxies (including early types S0 and S0/a; Gavazzi et al. 1996) results in total galaxy mass estimates of $6.3 \times 10^{10} M_{\odot}$ (SW) and $5.3 \times 10^{10} M_{\odot}(\mathrm{NE})$. Estimates of the stellar mass in old stars derived from the mean ratio of $M_{*} / \nu L_{v}(2.2 \mu \mathrm{m})=$ $5.6( \pm 1.5)$ based on model predictions by Lacey et al. (2008) produce similar results of $M_{*}=6.1 \times 10^{10} M_{\odot}(\mathrm{SW})$ and $M_{*}=4.4 \times 10^{10} M_{\odot}(\mathrm{NE}) .{ }^{24} \mathrm{SED}$ model fitting can improve on stellar masses estimated from monochromatic luminosities (U et al. 2012). Next, we turn attention to the nuclei and their circumnuclear regions.

\subsection{The Nuclei and Circumnuclear Regions}

\subsubsection{Optical and Infrared Spectral Diagnostics}

Using optical emission-line diagnostics, the NE nucleus has been classified as a LINER and the SW nucleus as a Seyfert 2 (e.g., Hutchings et al. 1988; Mazzarella \& Boroson 1993; Ishigaki et al. 2000). At optical wavelengths, the NE nucleus has brighter continuum emission and less extinction than the SW nucleus. Yuan et al. (2010) classified Mrk $266 \mathrm{NE}$ as a composite AGN/starburst nucleus, and they concluded that the NE and SW nuclei have similar relative AGN and starburst contributions within the LINER and Seyfert 2 branches of the [O III] 5007/ $H \beta$ versus [O I]6300/H $\alpha$ diagnostic diagram. ${ }^{25}$ Since the IRS apertures encompass an area of $5.4 \times 5.4 \mathrm{kpc}^{2}$ (Figure 10), whereas published optical diagnostics were obtained through smaller apertures of $\sim 1 \times 1 \mathrm{kpc}^{2}$, nuclear emission is more diluted by extranuclear emission in the mid-IR spectra than in the optical spectra. However, since the midIR spectra penetrate much more dust than the optical spectra, if there is sufficient centrally concentrated dust heated by an embedded AGN, this emission can potentially overpower the extended star formation (as in many ULIRGs). The IRS spectra of the two nuclei (Figure 12) show them to be similarly dominated by PAH emission, and the large PAH equivalent widths (Table 3) indicate the prominence of star formation (e.g., Armus et al. 2007). The NE source has lower PAH fluxes and equivalent widths, indicating excess warm dust emission compared to the SW source.

\footnotetext{
24 The model curve for $z=0$ galaxies in Figure 13(c) of Lacey et al. (2008), adjusted to $h=0.70$, corresponds to a range of 4.5-7.0 in $M_{*} / v L_{v}(2.2 \mu \mathrm{m})$. A significantly lower ratio of $M_{*} / L_{2.2} \mu \mathrm{m} \approx 1$ was found by Arnouts et al. (2007) and Cole et al. (2001). The nature of this discrepancy is beyond the scope of this article.

25 The identifications of the NE and SW nuclei of Mrk 266 were mistakenly swapped in the tables and figures of Kim et al. (1995) and Veilleux et al. (1995), and thus also in Table 2 of Yuan et al. (2010). The identifications are corrected here.
} 


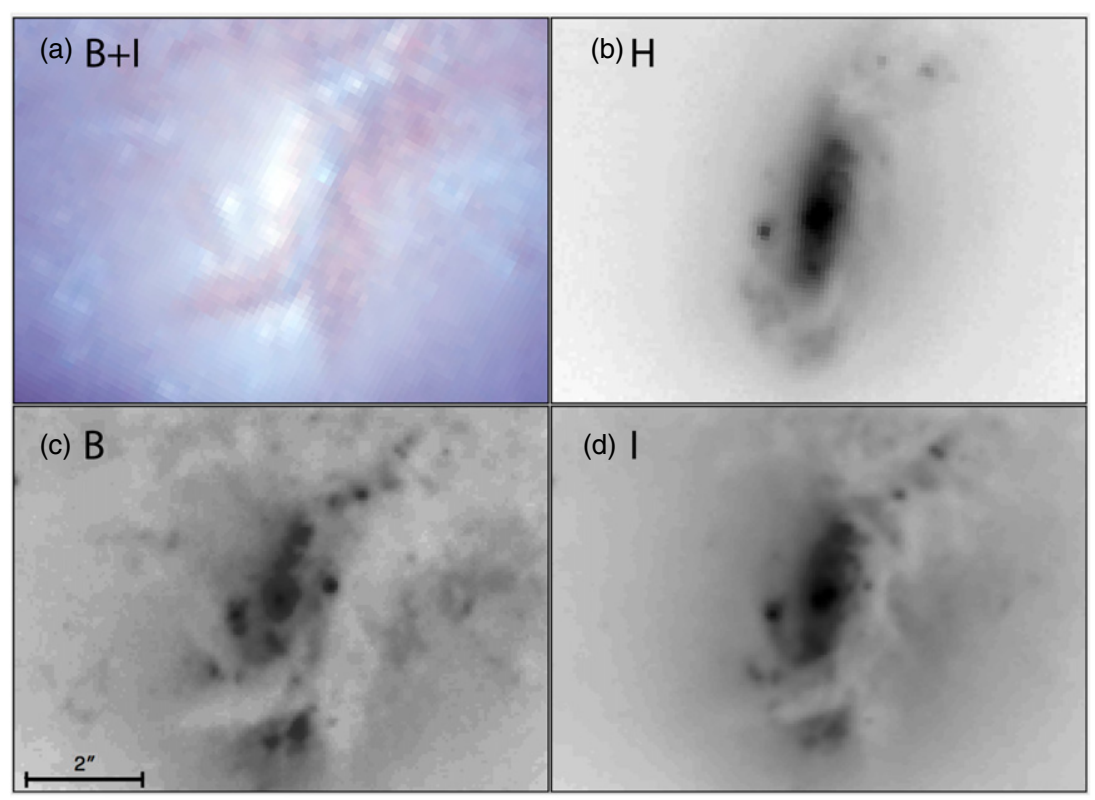

Figure 13. Close-up of $H S T$ imagery of Mrk 266 SW showing a color composite of (a) ACS $B+I$ bands, (b) NICMOS $H$ band, (c) ACS $B$ band, and (d) ACS $I$ band. The images are displayed with an emphasis on the high surface brightness emission near the nucleus. The spatial resolution is very similar in all four bands, but there has been no PSF matching. The scale bar indicates $2^{\prime \prime}$.

(A color version of this figure is available in the online journal.)

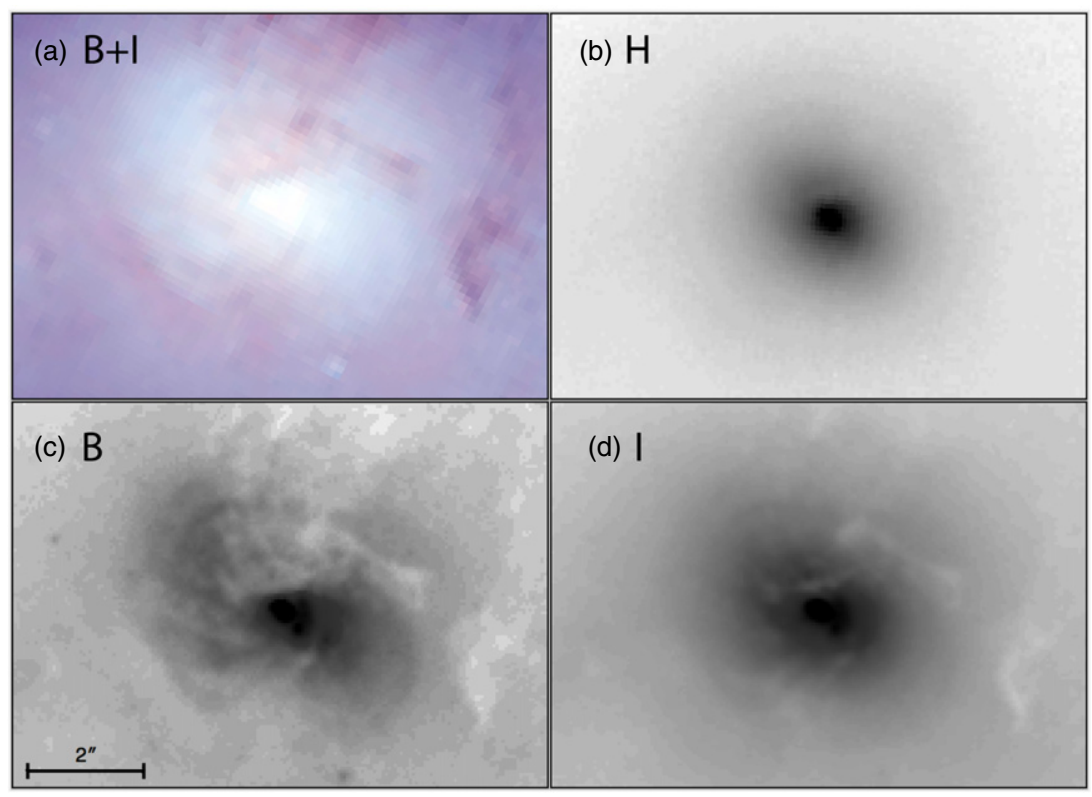

Figure 14. HST imagery of Mrk 266 NE displaying the same bands as in Figure 13.

(A color version of this figure is available in the online journal.)

The $6.2 \mu \mathrm{m}$ PAH EQWs and $9.7 \mu \mathrm{m}$ silicate absorption strengths ${ }^{26}$ place the SW galaxy on the bottom right vertex of the silicate depth versus PAH EQW plot of Spoon et al. (2007), a region dominated by galaxies with high star formation rates (SFRs); the NE galaxy (with similar Si strength but a lower $6.2 \mu \mathrm{m}$ EQW of $0.4 \mu \mathrm{m}$ compared to $0.67 \mu \mathrm{m}$ for the

\footnotetext{
${ }^{26}$ The empirical method of Spoon et al. (2007) was applied to the SL spectrum (5-14 $\mu \mathrm{m}$ region; Figure 12) to measure silicate strengths of $-0.62( \pm 0.06),-0.69( \pm 0.12)$, and $-0.66( \pm 0.07)$ for the SW nucleus, the NE nucleus, and the total system, respectively. A similar absorption strength, $-0.60( \pm 0.06)$, was measured using the $5-40 \mu \mathrm{m} \mathrm{SL}+\mathrm{LL}$ data for the total system (Figure 11).
}

SW galaxy) is in a region populated with AGNs and ULIRGs having substantial dust heating by non-stellar radiation. The observed silicate strength in the SW galaxy $(-0.60 \pm 0.06)$ is intermediate between the values observed for the face-on galaxy NGC 7714 and the edge-on galaxy M82, which is consistent with the derived disk axial ratio of $\approx 42^{\circ}$ for Mrk 266 SW (Table 4). The observed silicate absorption strengths correspond to $N_{\mathrm{H}}=$ $2.2 \times 10^{22}$ and $2.4 \times 10^{22} \mathrm{~cm}^{-2}$ for the SW and NE nuclei, respectively; these values result from applying the relationships $N_{\mathrm{H}}=\tau_{9.7 \mu \mathrm{m}} \times 3.5 \times 10^{22} \mathrm{~cm}^{-2}$ and $A_{9.7 \mu \mathrm{m}} / A_{v}=0.06$ (Roche \& Aitken 1984). However, for clumpy dust configurations expected in AGN tori, emission and absorption cannot be 


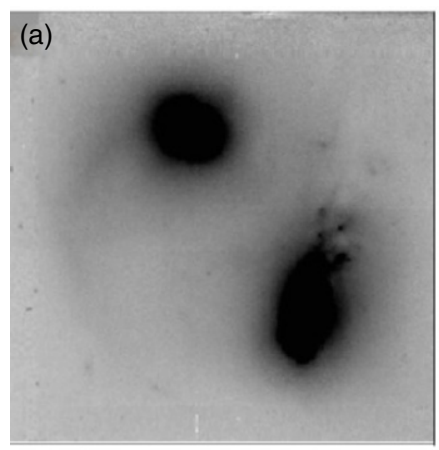

(d)

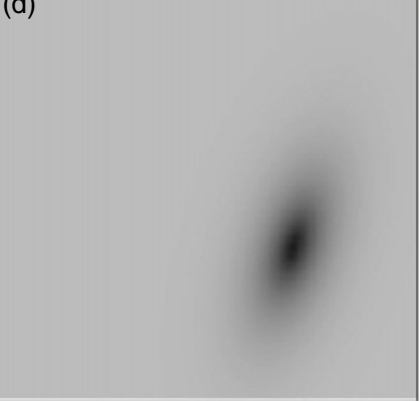

(g) (b)

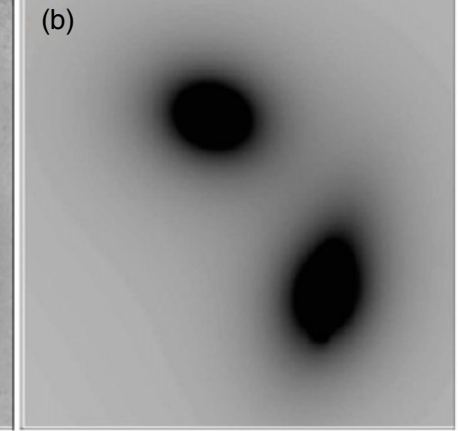

(e)

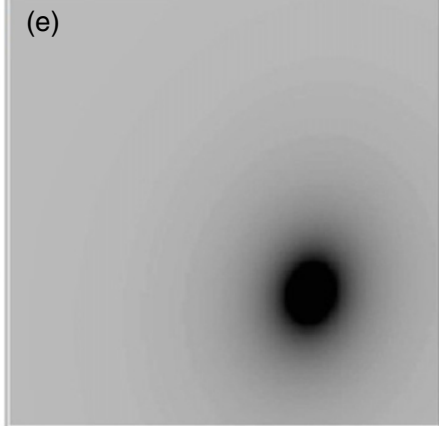

(h)

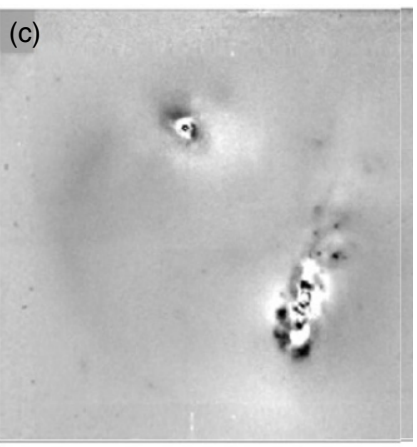

(f)

$10^{\prime \prime}(6 \mathrm{kpc})$

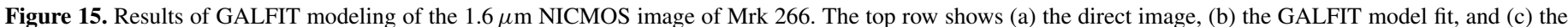

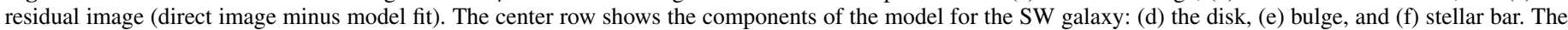

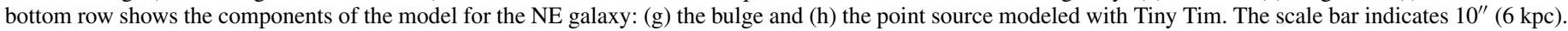

disentangled along the line of sight and therefore these are lower limits to the true silicate column for the central sources.

Various empirical diagnostics have been published to quantify the relative contributions to dust heating from AGN and starburst sources (e.g., Armus et al. 2007). We focus primarily on the suite of methods applied to local ULIRGs and QSOs by Veilleux et al. (2009), including application of their bolometric corrections to express the estimated AGN dust heating contributions as a fraction of $L_{\mathrm{bol}}$. All methods assume that the line-emitting regions are subject to the same amount of extinction within the aperture, which may be questionable, especially for Mrk 266 SW. Since the NE galaxy was not observed in the high-resolution IRS mode, methods that involve the fine structure lines can only be applied to the SW galaxy. The results are summarized in Table 5. For the SW galaxy, the $[\mathrm{OIV}] /[\mathrm{Ne}$ II] ratio indicates an overall AGN contribution to $L_{\mathrm{bol}}$ of $73 \%$, whereas the $[\mathrm{Ne} \mathrm{v}] /[\mathrm{Ne}$ II $]$ ratio suggests $53 \%$. The $6.2 \mu \mathrm{m}$ EQW indicates that the mid-IR luminosity of the SW component is due entirely to a starburst, and a $30 \%$ AGN contribution is inferred for the NE galaxy. This diagnostic suggests a $10 \%$ AGN contribution to the mid-IR luminosity of the total system. ${ }^{27}$ The AGN frac-

27 The CAFE SED fitting package provides an independent, model-dependent estimate of the AGN contribution to the total infrared luminosity. See Section 3.5 . tions estimated from the $6.2 \mu \mathrm{m}$ EQW are substantially lower than those obtained using the EQW of the $7.7 \mu \mathrm{m}$ PAH feature (see Table 5). This discrepancy may be due to greater difficulty measuring the $7.7 \mu \mathrm{m}$ PAH feature because of confusion from surrounding features ( $8.6 \mu \mathrm{m}$ PAH and silicate absorption) and complications in defining the proper continuum level.

The continuum shape over $3-16 \mu \mathrm{m}$ provides another diagnostic. The physical basis is that dust grains located near an AGN can reach temperatures as high as 500-1000 K (before they sublimate), and such high dust temperatures would completely dominate the continuum emission compared to dust heated by a starburst. This method was developed by Laurent et al. (2000), adapted by Peeters et al. (2004) for interpretation of mid-IR spectra from the Infrared Space Observatory (ISO), and modified further for Spitzer IRS data by Armus et al. (2007). Continuum fluxes in the relevant passbands were combined with the $6.2 \mu \mathrm{m}$ PAH fluxes, and the formulation of Veilleux et al. (2009) was used to compute AGN fractions before and after bolometric corrections. The results suggest $L_{\mathrm{AGN}} / L_{\text {bol }}$ is $33 \%$ for the NE galaxy, but only about $10 \%$ for the SW galaxy and the total system. The continuum flux density ratio $f_{v}(30 \mu \mathrm{m}) / f_{v}(15 \mu \mathrm{m})$ provides our final empirical diagnostic; Veilleux et al. (2009) found this to be an effective substitute for the PAH-free and silicate-free MIR/FIR ratio. 


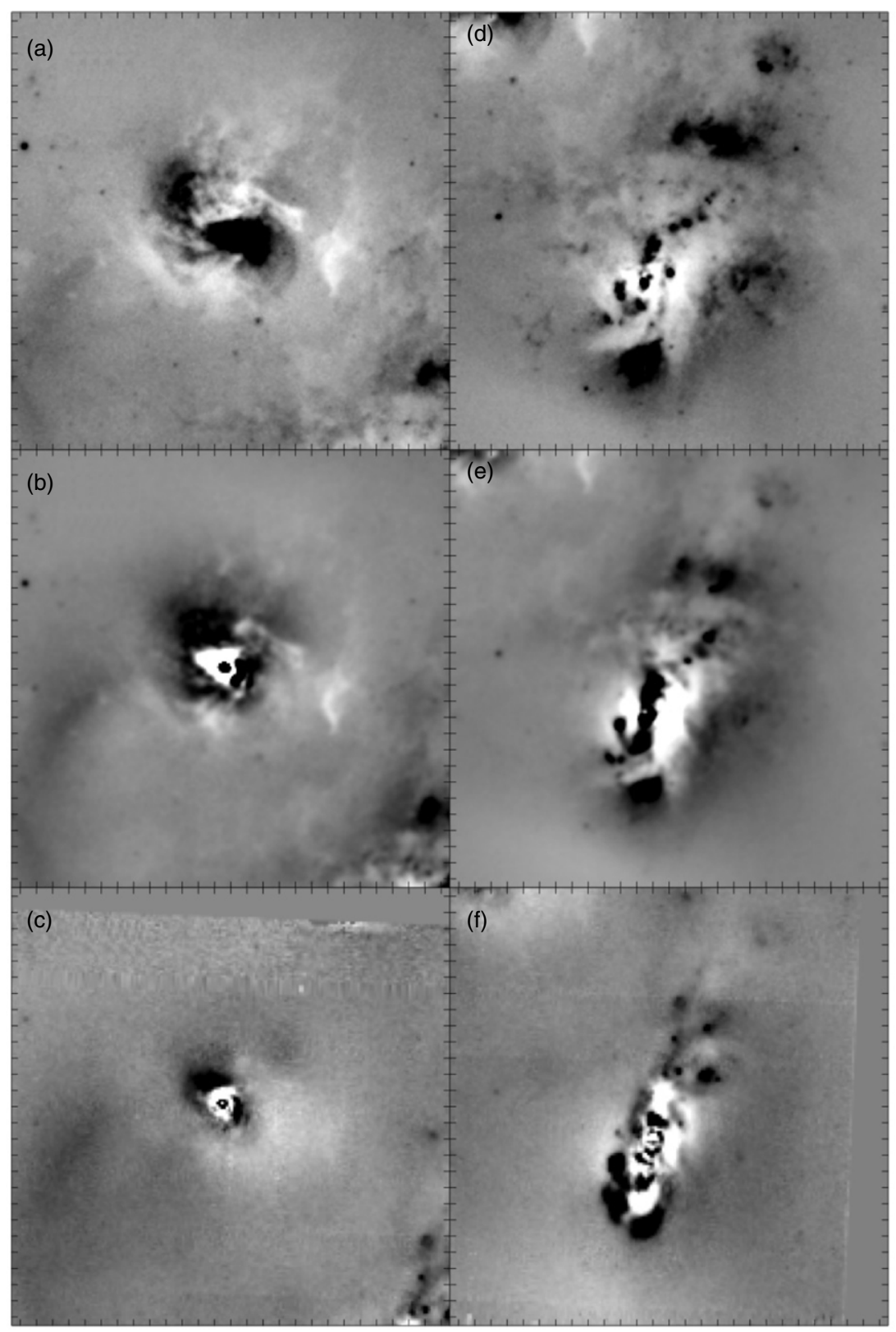

Figure 16. Residuals resulting from subtraction of GALFIT models parameterized in Table 4 from the direct $H S T$ images. The left column shows results for the NE galaxy at (a) $0.44 \mu \mathrm{m}$, (b) $0.81 \mu \mathrm{m}$, and (c) $1.6 \mu \mathrm{m}$. The right column shows results for the SW galaxy at (d) $0.44 \mu \mathrm{m}$, (e) $0.81 \mu \mathrm{m}$, and (f) $1.6 \mu \mathrm{m}$. Black (white) regions represent positive (negative) flux with respect to the image model fits. The tick marks are separated by 0.5.

On average, with a standard deviation of nearly $30 \%$, these diagnostics indicate approximately equal contributions of AGN and starburst heating of the dust in both galaxies (Table 5). This is similar to the average AGN contribution to $L_{\mathrm{bol}}$ found for ULIRGs in the QUEST sample with Seyfert 2 spectral classifications (Veilleux et al. 2009). The variation in these diagnostics may be due, in part, to the different optical depths probed by different wavelengths.

Finally, there is a significant difference in the PAH properties of the galaxies. The modeling of Draine \& Li (2001) combined with the ratios of the PAH features $\left(f_{11.3 \mu \mathrm{m}} / f_{7.7 \mu \mathrm{m}}\right.$ and $\left.f_{6.2 \mu \mathrm{m}} / f_{7.7 \mu \mathrm{m}}\right)$ indicate a dominance of relatively warm, ionized PAHs in the SW component and cooler, neutral PAHs in the NE component. The PAH line flux ratios for the total system reflect the fact that the SW galaxy dominates the total mid- and far-infrared emission from Mrk 266. The $f_{11.3 \mu \mathrm{m}} / f_{7.7 \mu \mathrm{m}} \mathrm{PAH}$ ratio in Mrk $266 \mathrm{NE}(0.76)$ is substantially larger than the ratio in the SW galaxy (0.34). The former is similar to the ratios observed in other galaxies containing AGNs, and the latter is near the mean value $(0.25)$ observed in galaxies with pure starburst (H II) spectra (Smith et al. 2007). Following the inferences proposed by Smith et al., this suggests that the hard radiation field surrounding the partially obscured AGN in the NE galaxy may be preferentially destroying PAH molecules small enough to emit at $7.7 \mu \mathrm{m}$, thus increasing the $11.3 \mu \mathrm{m} / 7.7 \mu \mathrm{m}$ PAH ratio compared to the SW nucleus which has a more heavily obscured AGN and a higher SFR.

\subsubsection{Warm Molecular Gas}

In the NE galaxy, the IRS spectral maps (Figures 10 and 12) reveal bright rotational lines of molecular hydrogen, specifically $\mathrm{H}_{2} \mathrm{~S}(3) 9.7 \mu \mathrm{m}$ and $\mathrm{H}_{2} \mathrm{~S}(2) 12.3 \mu \mathrm{m}$. In the $\mathrm{SW}$ galaxy, the $\mathrm{H}_{2} \mathrm{~S}(3) 9.7 \mu \mathrm{m}$ emission is much weaker, and any $\mathrm{H}_{2} \mathrm{~S}(2) 12.3 \mu \mathrm{m}$ emission that may be present intrinsically is 
Table 4

GALFIT Results

\begin{tabular}{|c|c|c|c|c|c|c|c|}
\hline Band & $\begin{array}{l}\frac{f}{f_{\text {tot }}} \\
(\%)\end{array}$ & $\mathrm{C}(\mathrm{n})$ & $\frac{B}{D}$ & $r_{e}$ & $\frac{b}{a}$ & P.A.$^{\circ}$ & $\operatorname{mag}_{A B}$ \\
\hline (1) & $(2)$ & (3) & (4) & (5) & (6) & (7) & (8) \\
\hline \multicolumn{8}{|c|}{ Mrk $266 \mathrm{SW}^{\mathrm{a}}$} \\
\hline $0.44 \mu \mathrm{m}$ & 68.2 & $\mathrm{~B}(4)$ & $\ldots$ & 9.99 & 0.79 & -38 & $\ldots$ \\
\hline $0.44 \mu \mathrm{m}$ & 68.2 & $\mathrm{~T}$ & $\ldots$ & $\ldots$ & $\ldots$ & $\ldots$ & $14.40 \pm 0.02$ \\
\hline $0.81 \mu \mathrm{m}$ & 63.0 & $\mathrm{~B}(4)$ & $\ldots$ & 8.28 & 0.79 & -19 & $\ldots$ \\
\hline $0.81 \mu \mathrm{m}$ & 63.0 & $\mathrm{~T}$ & $\ldots$ & $\ldots$ & $\ldots$ & $\ldots$ & $13.13 \pm 0.01$ \\
\hline $1.6 \mu \mathrm{m}$ & 41.8 & $\mathrm{~B}(4)$ & $\ldots$ & 4.92 & 0.49 & -13 & $\ldots$ \\
\hline $1.6 \mu \mathrm{m}$ & 9.6 & $\mathrm{D}(1)$ & 0.23 & 2.45 & 0.74 & -88 & $\ldots$ \\
\hline $1.6 \mu \mathrm{m}$ & 2.4 & $\mathrm{~b}$ & $\ldots$ & 0.57 & 0.27 & -9 & $\ldots$ \\
\hline $1.6 \mu \mathrm{m}$ & 0.0 & $\mathrm{P}$ & $\ldots$ & $\ldots$ & $\ldots$ & $\ldots$ & $\ldots$ \\
\hline $1.6 \mu \mathrm{m}$ & 53.8 & $\mathrm{~T}$ & $\ldots$ & $\ldots$ & $\ldots$ & $\ldots$ & $12.55 \pm 0.03$ \\
\hline \multicolumn{8}{|c|}{ Mrk $266 \mathrm{NE}^{\mathrm{b}}$} \\
\hline $0.44 \mu \mathrm{m}$ & 31.8 & $\mathrm{D}(1)$ & $\ldots$ & 3.11 & 0.70 & -91 & $\ldots$ \\
\hline $0.44 \mu \mathrm{m}$ & 31.8 & $\mathrm{~T}$ & $\ldots$ & $\ldots$ & $\ldots$ & $\ldots$ & $15.23 \pm 0.02$ \\
\hline $0.81 \mu \mathrm{m}$ & 13.9 & $\mathrm{~B}(4)$ & $\ldots$ & 1.43 & 0.60 & -113 & $\ldots$ \\
\hline $0.81 \mu \mathrm{m}$ & 23.1 & $\mathrm{D}(1)$ & 0.58 & 3.98 & 0.69 & -63 & $\ldots$ \\
\hline $0.81 \mu \mathrm{m}$ & 37.0 & $\mathrm{~T}$ & $\ldots$ & $\ldots$ & $\ldots$ & $\ldots$ & $13.71 \pm 0.02$ \\
\hline $1.6 \mu \mathrm{m}$ & 46.1 & $\mathrm{~B}(4)$ & $\ldots$ & 3.46 & 0.81 & -113 & $\ldots$ \\
\hline $1.6 \mu \mathrm{m}$ & 0.2 & $\mathrm{P}$ & $\ldots$ & 0.00 & 1.00 & 0 & $\ldots$ \\
\hline $1.6 \mu \mathrm{m}$ & 46.3 & $\mathrm{~T}$ & $\ldots$ & $\ldots$ & $\ldots$ & $\ldots$ & $12.71 \pm 0.03$ \\
\hline \multicolumn{8}{|c|}{ Mrk 266 Total $(\mathrm{SW}+\mathrm{NE})^{\mathrm{c}}$} \\
\hline $0.44 \mu \mathrm{m}$ & 1.0 & $\mathrm{~T}$ & $\ldots$ & $\ldots$ & $\ldots$ & $\ldots$ & $13.97 \pm 0.02$ \\
\hline $0.81 \mu \mathrm{m}$ & 1.0 & $\mathrm{~T}$ & $\ldots$ & $\ldots$ & $\ldots$ & $\ldots$ & $12.60 \pm 0.02$ \\
\hline $1.6 \mu \mathrm{m}$ & 1.0 & $\mathrm{~T}$ & $\ldots$ & $\ldots$ & $\ldots$ & $\ldots$ & $11.88 \pm 0.04$ \\
\hline
\end{tabular}

Notes. Fitting parameters and results of modeling the HST data using GALFIT. Column 1: HST filter: $B=\mathrm{F} 435 \mathrm{~W}(0.44 \mu \mathrm{m}) ; I=\mathrm{F} 814 \mathrm{~W}$ $(0.81 \mu \mathrm{m}) ; H=\mathrm{F} 160 \mathrm{~W}(1.6 \mu \mathrm{m})$. Column 2: percentage of the total flux of the GALFIT model of the galaxy pair accounted for by the indicated component of the model image at $0.44,0.81$, or $1.6 \mu \mathrm{m}$. Column 3: model components coded as follows: $B$ : bulge (Sérsic index $n=4$ ); $D$ : disk (Sérsic index $n=1$ ); $b$ : bar; $P$ : point source based on the Tiny Tim package (Krist 1993); $T$ : total flux from all GALFIT components for the galaxy. Column 4: bulge-to-disk ratio. Column 5: effective radius of the Sérsic fit (kpc units). Column 6: axis ratio of the component fit. Column 7: position angle of the component fit (degrees east of north). Column 8: apparent AB magnitude of the sum of the GALFIT model components for each galaxy, where $f_{v}(\mathrm{Jy})=10^{0.4\left(8.9-m_{A B}\right)}$. Conversions between the $\mathrm{AB}$ and Vega systems (http://www.stsci.edu/hst/acs/analysis/zeropoints/) are as follows: $B_{\mathrm{AB}}-B_{\mathrm{Vega}}=-0.11 ; I_{\mathrm{AB}}-I_{\mathrm{Vega}}=0.42 ; H_{\mathrm{AB}}-H_{\mathrm{Vega}}=1.32$.

${ }^{\text {a }}$ The $1.6 \mu \mathrm{m}$ image of Mrk $266 \mathrm{SW}$ is modeled as the sum of Point source + Bar + Disk $(\mathrm{n}=1)+$ Bulge $(\mathrm{n}=4)$ components. The 0.44 and $0.81 \mu \mathrm{m}$ images are too confused by extinction and $\mathrm{H}$ II regions to derive reliable models of the underlying stars; simple Bulge $(\mathrm{n}=4)$ models were fit at these wavelengths only to examine the residual images for fine structure in the star-forming regions (Figure 16).

${ }^{b}$ The $1.6 \mu \mathrm{m}$ image of Mrk 266 NE is modeled as the sum of Point source + Bulge $(n=4)$ components; there are no significant bar or disk components detected for this galaxy.

${ }^{\mathrm{c}}$ The total flux (apparent magnitude) accounted for in the GALFIT model for the galaxy pair in each HST passband.

overshadowed by the blue wing of the strong $12.6 \mu \mathrm{m}$ PAH complex. In contrast, the SW galaxy has bright [S IV] $10.51 \mu \mathrm{m}$ emission, whereas the NE nucleus is undetected in this feature. Given that the NE galaxy has 8 and $24 \mu \mathrm{m}$ flux densities that are 1.8 and 5.7 times fainter than the SW galaxy (see Table 13 and Figure 9), it is surprising that the $\mathrm{H}_{2}$ line emission in Mrk $266 \mathrm{NE}$ is substantially more luminous than in Mrk 266 SW. This suggests that the majority of the detected $\mathrm{H}_{2}$ line emission in the NE galaxy has a different origin than in the SW galaxy. A prime candidate for the enhanced $\mathrm{H}_{2}$ emission in Mrk $266 \mathrm{NE}$ is shock excitation.

Analysis of near-infrared spectra of Mrk 266 led Davies et al. (2000) to conclude that fast C shocks $\left(v_{s} \sim 40 \mathrm{~km} \mathrm{~s}^{-1}\right)$ are responsible for $\approx 70 \%$ of the $\mathrm{H}_{2} \quad 1-0 \mathrm{~S}(1)$ emission in the two nuclei, with fast $\mathrm{J}$ shocks $\left(v_{s} \sim 300 \mathrm{~km} \mathrm{~s}^{-1}\right)$ and UV fluorescence responsible for the remaining $\approx 30 \%$. Davies et al. demonstrated that the powerful radio continuum and 1-0 S(1) emissions observed in Mrk $266 \mathrm{NE}$ cannot be explained with pure starburst models, and they are likely generated predominantly by shocks. Furthermore, the presence of 1-0 S(1) emission extended out to $\sim 1 \mathrm{kpc}$ from the NE nucleus, with a large equivalent width and low $\mathrm{S}(1) / \mathrm{B} \gamma$ ratio that rule out star formation, provides additional evidence that the $\mathrm{H}_{2}$ emission is excited by spatially extended shocks. Davies et al. speculated that Mrk 266 NE may itself be a very close pair of galactic nuclei with shocked molecular gas settled between them, as observed in NGC 6240. Our interpretation of the latest data from Spitzer, $H S T$, and Chandra favors an AGN-driven outflow as the origin of strong shocks in Mrk $266 \mathrm{NE}$ (see Section 3.2.4).

\subsubsection{AGN Black Hole Masses}

Marconi \& Hunt (2003) established an empirical relation between $H$-band bulge luminosity and nuclear black hole mass. Combining measurements of the total $H$-band absolute magnitudes with the GALFIT image decomposition results showing that $78 \%$ and $99 \%$ of the total $H$-band light is in the bulge components of the SW and NE galaxies (Section 3.1.1), the calibration of Marconi \& Hunt (Group 1 Galaxies) yields black hole masses of $2.3 \times 10^{8}(\mathrm{SW})$ and $2.6 \times 10^{8} M_{\odot}(\mathrm{NE})$. An independent diagnostic is available from the high-ionization, 
Table 5

Estimated AGN Contributions to the Mid-infrared and Bolometric Luminosity

\begin{tabular}{|c|c|c|c|c|c|c|c|c|c|}
\hline \multirow[t]{2}{*}{ Diagnostic } & \multicolumn{3}{|c|}{ SW Galaxy } & \multicolumn{3}{|c|}{ NE Galaxy } & \multicolumn{3}{|c|}{ Total System } \\
\hline & $\begin{array}{c}\text { Value } \\
\text { (2) }\end{array}$ & $\begin{array}{c}\frac{L_{\mathrm{AGN}}}{L_{\mathrm{Diag}}} \\
(3)\end{array}$ & $\begin{array}{c}\frac{L_{\mathrm{AGN}}}{L_{\mathrm{Bol}}} \\
(4)\end{array}$ & $\begin{array}{c}\text { Value } \\
(5)\end{array}$ & $\begin{array}{c}\frac{L_{\mathrm{AGN}}}{L_{\mathrm{Diag}}} \\
(6)\end{array}$ & $\begin{array}{c}\frac{L_{\mathrm{AGN}}}{L_{\mathrm{Bol}}} \\
(7)\end{array}$ & $\begin{array}{c}\text { Value } \\
(8)\end{array}$ & $\begin{array}{c}\frac{L_{\mathrm{AGN}}}{L_{\mathrm{Diag}}} \\
(9)\end{array}$ & $\begin{array}{l}\frac{L_{\mathrm{AGN}}}{L_{\mathrm{Bol}}} \\
(10)\end{array}$ \\
\hline (1) $\left[\mathrm{O}_{\mathrm{IV}}\right] 25.89 \mu \mathrm{m} /[\mathrm{Ne}$ II $] 12.81 \mu \mathrm{m}^{\mathrm{a}}$ & 0.93 & $23 \%$ & $73 \%$ & $\ldots$ & $\ldots$ & $\ldots$ & 0.67 & $17 \%$ & $64 \%$ \\
\hline (2) $[\mathrm{Ne} \mathrm{v}] 14.32 \mu \mathrm{m} /\left[\mathrm{Ne}\right.$ II] $12.81 \mu \mathrm{m}^{\mathrm{a}}$ & 0.14 & $11 \%$ & $53 \%$ & $\ldots$ & $\ldots$ & $\ldots$ & $\ldots$ & $\ldots$ & $\ldots$ \\
\hline (3) PAH $6.2 \mu \mathrm{m}$ EQW $[\mu \mathrm{m}]^{\mathrm{b}}$ & 0.67 & $0 \%$ & $\ldots$ & 0.4 & $30 \%$ & $\ldots$ & 0.47 & $10 \%$ & $\ldots$ \\
\hline (4) PAH $7.7 \mu \mathrm{m}$ EQW $[\mu \mathrm{m}]^{\mathrm{b}}$ & 0.54 & $69 \%$ & $43 \%$ & 0.25 & $79 \%$ & $56 \%$ & 0.46 & $71 \%$ & $46 \%$ \\
\hline (5) $f(\mathrm{PAH} 6.2 \mu \mathrm{m}) / f_{5.5} \mu \mathrm{m}$ vs. $f(15 \mu \mathrm{m}) / f_{5.5} \mu \mathrm{m}^{\mathrm{b}}$ & $1.6,8.6$ & $47 \%$ & $9 \%$ & $0.56,6.4$ & $81 \%$ & $33 \%$ & $2.0,2.9$ & $52 \%$ & $11 \%$ \\
\hline (6) $f_{v}(30) / f_{v}(15)^{\mathrm{c}}$ & 3.9 & $89 \%$ & $82 \%$ & 3.3 & $92 \%$ & $87 \%$ & 4.8 & $85 \%$ & $76 \%$ \\
\hline Mean $^{\mathrm{d}}$ & & $40 \%$ & $52 \%$ & & $71 \%$ & $59 \%$ & & $47 \%$ & $49 \%$ \\
\hline Std. Dev. & & $35 \%$ & $29 \%$ & & $28 \%$ & $27 \%$ & & $33 \%$ & $28 \%$ \\
\hline
\end{tabular}

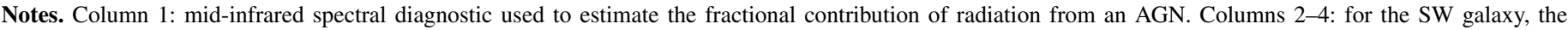

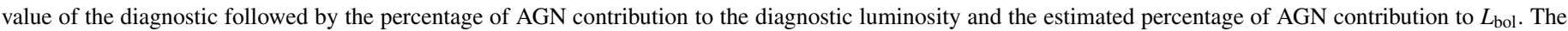

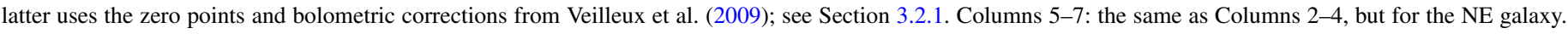
Columns 8-10: the same as Columns 2-4, but for the Total System.

${ }^{a}$ Based on published high-resolution, staring-mode data (Table 14). The NE nucleus was not fully covered in these observations.

b Based on new low-resolution measurements from the IRS spectral map (Table 3).

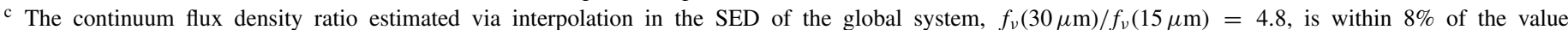

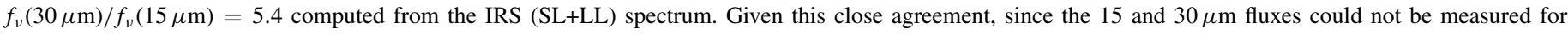

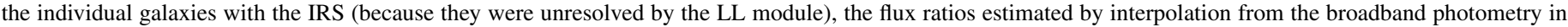
the SEDs for the individual galaxies (Table 13) were substituted.

d The mean $L_{\mathrm{AGN}} / L_{\mathrm{Bol}}$ values omit the $6.2 \mu \mathrm{m}$ PAH EQW because there is no bolometric correction available for this diagnostic.

Table 6

Photometry of Knots Embedded in the Arc 0.'4 West of the NE Nucleus

\begin{tabular}{|c|c|c|c|}
\hline $\begin{array}{l}\text { R.A. } \\
\text { (hh:mm:ss.sss) } \\
\text { (1) }\end{array}$ & $\begin{array}{c}\text { Decl. } \\
\text { (dd:mm:ss.ss) } \\
\text { (2) }\end{array}$ & $\begin{array}{c}m_{\mathrm{F} 435 \mathrm{~W}} \\
(\mathrm{mag}) \\
(3)\end{array}$ & $\begin{array}{c}m_{\mathrm{F} 814 \mathrm{~W}} \\
(\mathrm{mag}) \\
(4)\end{array}$ \\
\hline $13: 38: 17.75$ & $48: 16: 40.9$ & $19.94 \pm 0.03$ & $19.02 \pm 0.05$ \\
\hline $13: 38: 17.75$ & $48: 16: 41.1$ & $20.09 \pm 0.03$ & $18.89 \pm 0.04$ \\
\hline $13: 38: 17.75$ & $48: 16: 41.3$ & $20.27 \pm 0.03$ & $19.06 \pm 0.04$ \\
\hline
\end{tabular}

Notes. Columns 1 and 2: J2000 R.A. and decl., with $1 \sigma$ uncertainty in the absolute astrometry of 0.5 . Columns 3 and 4: apparent magnitudes and uncertainties measured in the HST/ACS F435W (B) and F814 (I) bands, respectively.

mid-IR emission lines detected in Mrk 266 SW. Using the approach of Dasyra et al. (2008), the [Ne v] $14.32 \mu \mathrm{m}$ $\left(\left[\mathrm{O}_{\mathrm{IV}}\right] 25.89 \mu \mathrm{m}\right)$ emission line luminosity of $1.58(10.5) \times$ $10^{41} \mathrm{erg} \mathrm{s}^{-1}$ computed from published high-resolution IRS measurements (Table 14) corresponds to $M_{B H}=1.7(3.2) \times 10^{8} M_{\odot}$ for the SW nucleus, which is consistent with the IR photometry. The inferred black hole masses are therefore in the typical range known to power other luminous AGNs.

\subsubsection{Evidence for Nuclear Outflows and Shocks}

In the previous section, the radiation field heating the dust in the two nuclear environments was established. In this section, evidence is presented for dynamical outflows from the nuclei. Within a radius of $2^{\prime \prime}(1.2 \mathrm{kpc})$ around the NE nucleus (Figures 14(c) and (d) and Figure 16(a)), the HST optical imaging reveals structure that is strongly suggestive of a bi-conical outflow with patchy dust extinction. Evidence for this interpretation rather than spiral arms is as follows. (1) Multiple filamentary structures extend radially and asymmetrically from the nucleus rather than in a symmetric spiral pattern. (2) There is the lack of improved definition in any spiral arm pattern in the $I$-band image relative to the $B$-band. (3) Photometric deconvolution
(Section 3.1.1) indicates that the NE galaxy is bulge-dominated. There are also conspicuous knots of concentrated emission along a concave arc located $0.4 \mathrm{~W}$ of the nucleus. Table 6 lists photometric measurements of the three most prominent optical knots. The individual knot luminosities of $L_{B} \approx 3 \times 10^{8} L_{\odot}$ are substantially higher than other luminous SCs in Mrk 266 (see Section 3.6, Figure 27), and their orientation in an arc embedded within a triangular bow-like structure suggests they may be energized by an AGN-driven outflow in a situation similar to M51 (Cecil 1988) rather than by unusually massive SCs.

A comparison of the $B$-band emission with published highresolution $18 \mathrm{~cm}$ radio continuum observations (Thean et al. 2001) is shown in Figure 17. The $18 \mathrm{~cm}$ MERLIN map exhibits limb brightening at the location of the optical arc and knots 0.4 (240 pc) west of the nucleus, and a secondary radio continuum peak is located 0.'6 (360 pc) NE of the nucleus. An axis at position angle $56^{\circ}$ intersects the radio/optical nucleus, the radio emission 0.'6 NE of the nucleus, and $B$-band knots situated 0.'4 and $0.9 \mathrm{SW}$ of the nucleus. Bi-conical AGN outflows with aligned radio continuum and optical emission-line radiation with dimensions of $\lesssim 1 \mathrm{kpc}$ are commonly observed in Seyfert galaxies and in some LINERS, especially those that are nearly face-on such as NGC 1068 and M51. (See, for example, the review of Veilleux et al. 2005.) The optical morphology of the NE nuclear environment is remarkably similar to the radiative bow shock observed $5^{\prime \prime}(230 \mathrm{pc})$ south of the nucleus of M51, which is also a LINER (Cecil 1988; Ford et al. 1985).

The combined data suggest that the AGN in Mrk $266 \mathrm{NE}$ is ejecting radio-emitting plasma along an axis with P.A. $\approx 56^{\circ}$, which is also aligned with a bi-conical ionization cone that extends to a radius of $1.2 \mathrm{kpc}$ from the nucleus. The $6 \mathrm{~cm}$ radio power $\left(4.2 \times 10^{22} \mathrm{~W} \mathrm{~Hz}^{-1}\right)$ and spectral index $\left(\alpha_{20,6,2 \mathrm{~cm}}=\right.$ $0.61 \pm 0.04)$ of Mrk $266 \mathrm{NE}$ are consistent with optically thin synchrotron emission (Mazzarella et al. 1988), and these parameters are similar to other luminous AGNs in the local universe (e.g., Rush et al. 1996). The radio jet appears to be running into dense material concentrated 0.'4 (240 pc) SW from 


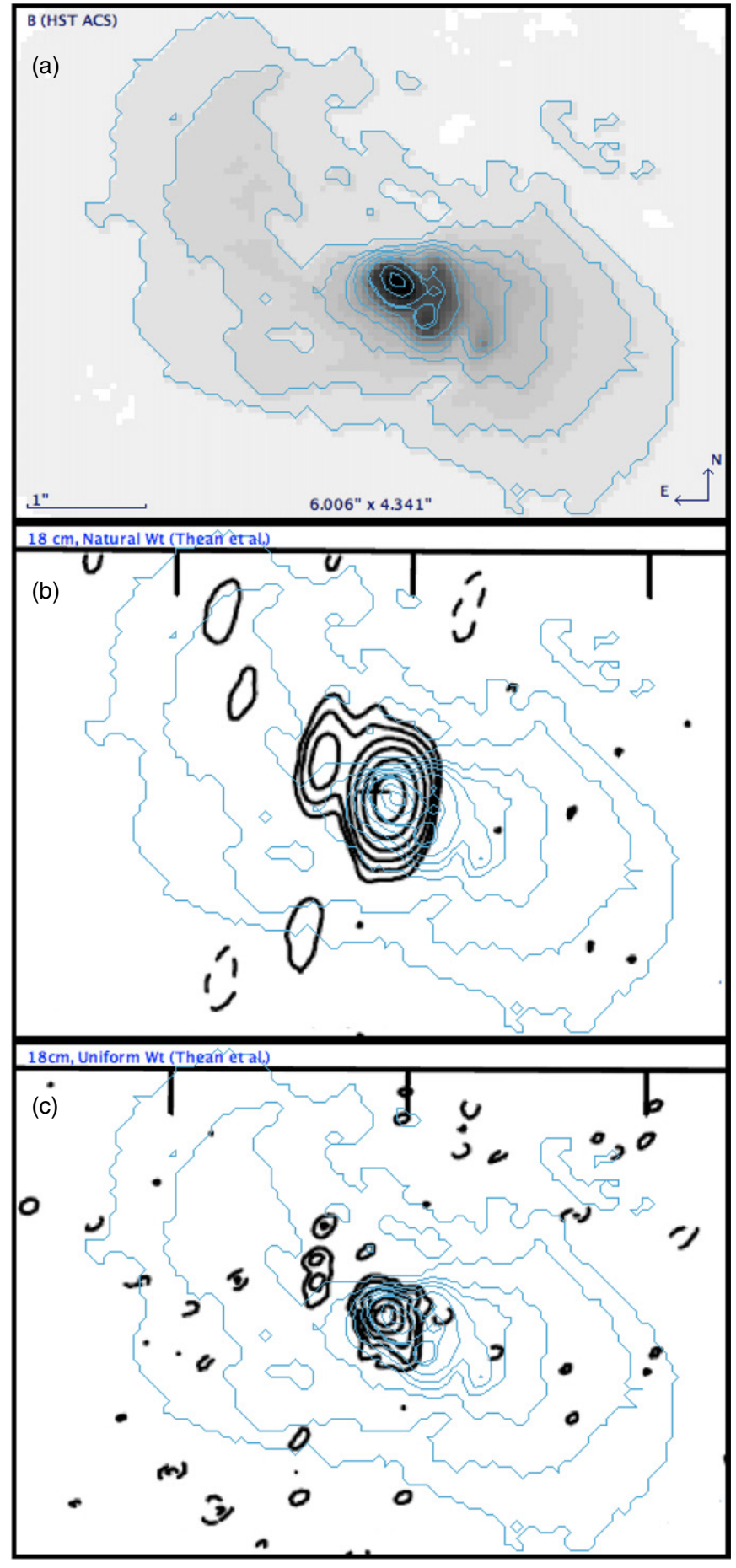

Figure 17. Comparison of new $0.44 \mu \mathrm{m}$ (HST ACS) imagery with published $18 \mathrm{~cm}$ radio continuum (MERLIN) emission (Thean et al. 2001, Figure 1) in the circumnuclear region of Mrk 266 NE. Top (a): HST ACS $B$-band image in gray scale; the same $B$-band data are contoured (blue) and overlaid here and in the following two panels. Center (b): $18 \mathrm{~cm}$ radio continuum interferometry with natural weighting and spatial resolution $0.5 \times 0.5$ HPBW (thick black contours) overlaid with ACS $B$ band (thin blue contours). Bottom (c): $18 \mathrm{~cm}$ radio continuum interferometry with uniform weighting and spatial resolution $0.2 \times 0.1$ HPBW (thick black contours) overlaid with ACS $B$ band (thin blue contours). The plus signs in panels (b) and (c) are from Thean et al. (2001, see their Figure 1) and they mark the optical nuclear positions, where the marker extent indicates the $2 \sigma$ positional uncertainty. These positions are in good agreement with the astrometry we derived for the ACS images. The scale bar indicates $1^{\prime \prime}$, and the field of view is 6 .' $0 \times 4$.' 3 for all three panels.

(A color version of this figure is available in the online journal.) the nucleus, but it is able to escape to a greater distance in the opposite direction (Figure 17). This hypothesis is consistent with the velocity field measured for the molecular gas (Section 3.7) which shows a rotating disk aligned roughly orthogonal to this putative ionization cone. The $B-I$ color map from the $H S T$ data (Figure 26) reveals that dust is distributed primarily along the NS direction, which again is roughly orthogonal to the AGN jet/outflow axis. Regions inside a radius of $\sim 2^{\prime \prime}$ from the nucleus of Mrk 266 NE (Figure 26) are as blue as the Northern Loop, which is known to be dominated by bright [O III] $\lambda 5007$ and $\mathrm{H} \alpha$ line emission (Hutchings et al. 1988; Ishigaki et al. 2000). ${ }^{28}$ Davies et al. (2000) provided independent evidence for shockheated gas in this region in the form of the radial profile of the 1-0 S(1) $\mathrm{H}_{2} 2.12 \mu \mathrm{m}$ emission line. Furthermore, the $\mathrm{Br} \gamma$ emission-line image presented by Davies et al. (2000) shows a bimodal structure oriented at P.A. $\approx 50^{\circ}$ centered on the NE nucleus, providing additional support for our hypothesis. While most of this evidence is indirect, its combined weight favors our interpretation that the extranuclear emission around Mrk $266 \mathrm{NE}$ is dominated by an AGN-powered outflow that is generating spatially extended shocks.

Similar events may be occurring in the SW galaxy. The HST images show numerous compact structures over a $1 \mathrm{kpc}$ size region that share the same NS orientation as the prominent stellar bar seen in the $H$-band image. It is highly likely that these compact structures are young stellar clusters (see Section 3.6). Observations at 1.6 GHz (Thean et al. 2001) show sub-arcsecond structure aligned NW-SE with respect to the SW nucleus, similar to previous $5 \mathrm{Ghz}$ measurements (Mazzarella et al. 1988). Comparing the new HST images with the extended radio emission maps suggests that the circumnuclear emission in this case is dominated by star formation. An AGN outflow in the SW galaxy may be present but is difficult to detect due to its relatively high inclination $\left(\approx 42^{\circ}\right)$. It is very difficult to disentangle starburst-driven winds from AGN-driven winds in galaxies containing both a Seyfert 2 and a powerful starburst (e.g., Veilleux et al. 2005). Although evidence for an AGNdriven outflow in the circumnuclear region of Mrk $266 \mathrm{SW}$ is weak, there is ample evidence for a starburst-driven superwind on a larger scale based on the filamentary $\mathrm{H} \alpha$ and [O III] line emission that comprise the Northern Loop, and from the fainter emission-line filaments labeled "eastern arm-like region" and "west knot" in Figure 2 of Ishigaki et al. (2000), all of which appear to connect or align with the SW nucleus. Properties of the kiloparsec-scale outflow revealed by the new data are discussed in Section 3.4.

\subsubsection{Hard X-Ray Properties of the Nuclei}

Basic properties of the Chandra data for Mrk 266 were presented by Brassington et al. (2007). We have performed additional processing to reveal previously unknown structural details and physical properties. As shown in Figure 18(a), the SW (Seyfert 2) nucleus has a much softer X-ray spectrum and lower luminosity than the NE (LINER or composite AGN/starburst) nucleus. The luminosity and hard X-ray spectrum of the NE nucleus provide a clear signature of an obscured

\footnotetext{
28 The [0 III] $\lambda 5007$ line emission around Mrk 266 NE (Hutchings et al. 1988) is elongated along the same position angle as the HST B-band image contoured here in Figure 17. This provides evidence that the HST B-band image is dominated by emission-line gas in this region. The passband of the F435W filter omits the [O III] lines, but it includes the [O II] $\lambda 3727$ line observed in the spectrum of Mrk $266 \mathrm{NE}$ (Kim et al. 1995) and also at large radii within the ionization cones of many AGNs (e.g., Tadhunter 2008).
} 

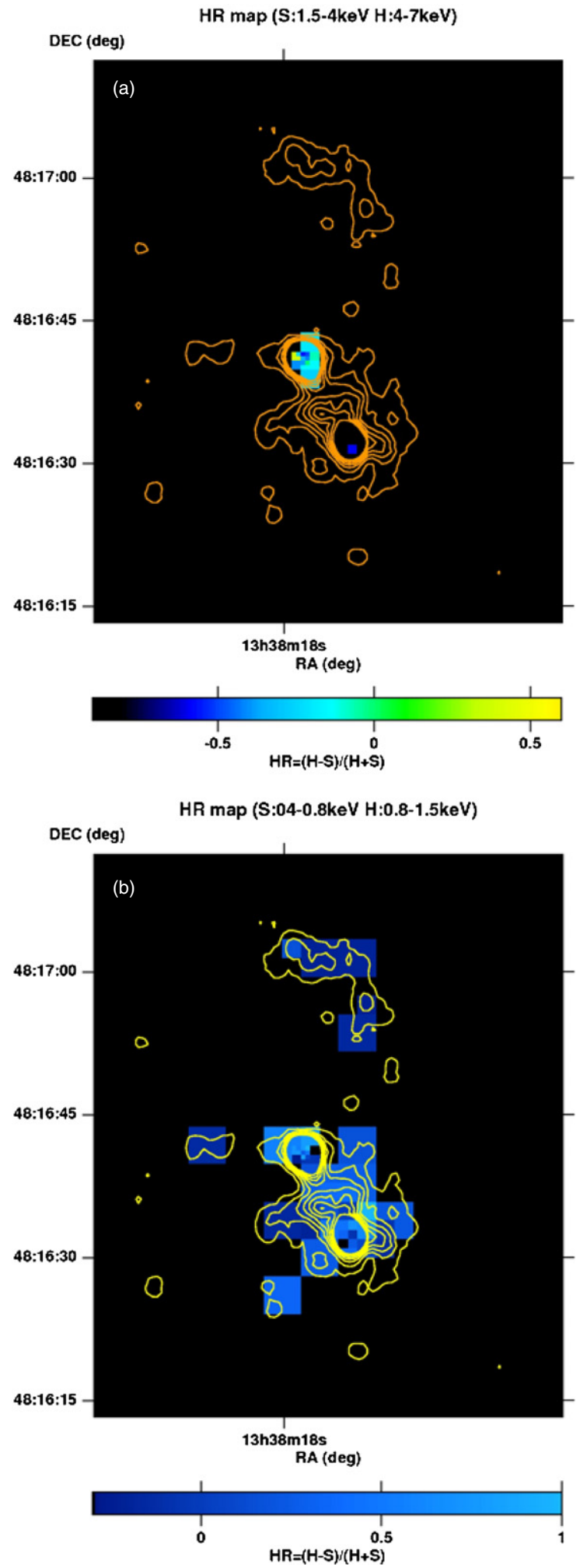

Figure 18. Maps of the hardness of the Chandra X-ray emission. (a) The hardness ratio between 1.5 and $7 \mathrm{keV}$, defined as $\mathrm{HR}_{\text {hard }}=\left(\mathrm{F}_{4-7 \mathrm{keV}}-\right.$ $\left.\mathrm{F}_{1.5-4 \mathrm{keV}}\right) /\left(\mathrm{F}_{4-7 \mathrm{keV}}+\mathrm{F}_{1.5-4 \mathrm{keV}}\right)$. The hard X-ray emission from the $\mathrm{SW}$ nucleus is too faint to measure this flux ratio, with the exception of the peak pixel which has $\mathrm{HR}_{\text {hard }}=-0.4$ (blue). (b) The hardness ratio between 0.4 and $1.5 \mathrm{keV}$, defined as $\mathrm{HR}_{\text {soft }}=\left(\mathrm{F}_{0.8}-1.5 \mathrm{keV}-\mathrm{F}_{0.4-0.8 \mathrm{keV}}\right) /\left(\mathrm{F}_{0.8}-1.5 \mathrm{keV}+\mathrm{F}_{0.4-0.8 \mathrm{keV}}\right)$; this illustrates the temperature variation over the diffuse emission. $\mathrm{HR}_{\text {hard }}$ and $\mathrm{HR}_{\mathrm{soft}}$ are plotted using colors as indicated in their respective legends. The soft band $\mathrm{X}$-ray data are overlaid as contours drawn with linear intervals between $1.4 \%$ and $12 \%$ of the peak brightness (in the NE nucleus) to emphasize the extended, diffuse emission.

(A color version of this figure is available in the online journal.)
AGN. The X-ray spectrum obtained of the integrated system with $X M M$ (Figure 6) shows strong $\mathrm{Fe} \mathrm{K} \alpha$ emission at $6.4 \mathrm{keV}$. This high-ionization feature has been observed in the ULIRG Arp 220 (Iwasawa et al. 2005), in the LIRG NGC 3690 (Ballo et al. 2004), and more recently in the integrated (stacked) spectrum of 30 LIRGs in the GOALS sample that are relatively faint in the hard X-ray band (Iwasawa et al. 2009). Modeling the global XMM spectrum of Mrk 266 with a thermal (soft, extended) component due to star formation and an absorbed power-law (hard, compact) component from the AGNs results in an absorption column of $N_{\mathrm{H}}=6.5 \times 10^{22} \mathrm{~cm}^{-2}$, which is close to the lower limits derived from the $9.6 \mu \mathrm{m}$ silicate absorption feature (Section 3.2.1).

Unlike XMM, Chandra was able to resolve the nuclei and has detected two separate sources of (2-7 keV) X-ray emission, with the bulk of the combined emission emanating from the NE component (Figure 3). The physical origin of the bright Fe $\mathrm{K} \alpha$ line observed in the $X M M$ spectrum of the integrated system is less clear. The Chandra spectrum of the total emission from Mrk 266 (Figure 5(f)) does not show the $\mathrm{Fe} \mathrm{K} \alpha$ line. However, the large aperture ( $33^{\prime \prime}$ radius) used to produce this spectrum introduces substantial noise in the background in the higher energy bands where there is very little extended emission. Detailed inspection of the Chandra data within small apertures centered on the nuclei provides a strong clue that the $\mathrm{Fe} \mathrm{K} \alpha$ line originates primarily from the SW nucleus, ${ }^{29}$ indicative of a reflection-dominated spectrum of a heavily obscured AGN. Since optical spectra reveal the presence of Seyfert 2 activity in the SW nucleus, the weakness of the hard X-ray emission can be understood as a consequence of suppression of X-rays by Compton thick absorption. Heavy obscuration toward the SW nucleus as inferred from the Spitzer IRS spectrum and the large column density of obscuring material estimated from the $\mathrm{CO}$ (1-0) observations (Section 3.7) support this interpretation of the X-ray data.

If the 6-7 keV photons in the Chandra spectrum of the SW nucleus are due to $\mathrm{Fe} \mathrm{K} \alpha$ line emission, as our analysis suggests, the flux of $1.2_{-0.5}^{+0.7} \times 10^{-6}$ photons $\mathrm{cm}^{-2} \mathrm{~s}^{-1}$ accounts for the majority of the Fe $\mathrm{K} \alpha$ line flux detected in the XMM-Newton spectrum. This means that the NE nucleus accounts for most of the escaping hard X-ray continuum, but it emits little Fe K emission. The equivalent width of $\mathrm{Fe} \mathrm{K}$ expected from a source absorbed by $N_{\mathrm{H}} \approx(6-8) \times 10^{22} \mathrm{~cm}^{-2}$, as inferred for the $\mathrm{NE}$ nucleus (Table 2), is at most $0.1 \mathrm{keV}$ (e.g., Awaki et al. 1991; Krolik et al. 1994; Ghisellini et al. 1994), while the equivalent width measured in the XMM-Newton spectrum is $\sim 0.5 \mathrm{keV}$. This mismatch can be readily explained if the bulk of the Fe $\mathrm{K} \alpha$ line emission is from the $\mathrm{SW}$ nucleus. The $\mathrm{Fe} \mathrm{K} \alpha$ line luminosity is $3 \times 10^{40} \mathrm{erg} \mathrm{s}^{-1}$. Using the relation derived in Iwasawa et al. (2005) for the Type $2 \mathrm{AGN}$ in the HyLIRG IRAS $\mathrm{F} 15307+3252$, the intrinsic 2-10 keV luminosity of the obscured AGN in Mrk $266 \mathrm{SW}$ is $\sim 1 \times 10^{42} f^{-1} \mathrm{erg} \mathrm{s}^{-1}$, where $f$ is the (unknown) visible fraction of the reflecting matter in the line of sight. The observed $F_{x} / F_{\left[\mathrm{O}_{\text {III }}\right]}$ ratio for the $\mathrm{SW}$ nucleus also indicates a highly absorbed X-ray source with $N_{\mathrm{H}} \gtrsim 10^{24} \mathrm{~cm}^{-2}$ (see Appendix A.3).

\footnotetext{
29 The 6-7 keV (Fe K) image contains 10 counts from the NE nucleus and 4 counts from the SW nucleus, while the neighboring 3-6 keV band shows a much larger contrast, with 79 counts from the NE nucleus and only 1 count from the SW nucleus. In other words, the spectrum of the SW nucleus has almost no detected flux at energies $>4 \mathrm{keV}$, but it shows a sudden rise at the Fe K band (Figure 5). This suggests that the hard band spectrum of the SW nucleus is dominated by flux from the $\mathrm{Fe} \mathrm{K} \alpha$ line.
} 
Finally, it should be emphasized that the $\mathrm{Fe} \mathrm{K} \alpha$ line detections are marginal. However, we gain confidence through independent $2 \sigma$ detections for the global system with $X M M$ and for the SW galaxy with Chandra, combined with line flux values that agree within the uncertainties in the calibrations and $\mathrm{S} / \mathrm{N}$ (Table 2). A detection of Fe has also been reported at the $90 \%$ confidence level with ASCA GIS observations (Risaliti et al. 2000). Confirmation requires more sensitive observations.

\subsubsection{The LINER in Mrk 266 NE}

The relative strength of the PAH features, silicate absorption, and continuum shape indicates that Mrk 266 NE is typical of an "IR LINER" or "Transition" between IR-luminous LINERs and "Type 1" LINERs. Although the mid-IR data for Mrk $266 \mathrm{NE}$ are only sufficient to compute 3 of the 6 parameters in diagnostic diagrams (Figure 2 of Sturm et al. 2006), the relatively small $6.2 \mu \mathrm{m} / 11.2 \mu \mathrm{m}$ PAH flux ratio (1.1) is on the low end of the distribution observed in "IR LINERs" (where the transition to Type 1 occurs), whereas the continuum flux ratios $f_{v}(15 \mu \mathrm{m}) / f_{v}(6 \mu \mathrm{m})=16$ and $f_{v}(30 \mu \mathrm{m}) / f_{v}(6 \mu \mathrm{m})=54$ place Mrk 266 NE on the extreme end of the distribution of "IR LINERs" with very red, warm dust continua. Mid-IR spectral data alone do not permit a clear distinction between the various kinds of energy sources that can power a LINER (e.g., Kewley et al. 2006; Ho 2008). However, there are at least four indicators that Mrk $266 \mathrm{NE}$ is energized primarily by an AGN, in addition to shock excitation: (1) small PAH equivalent widths and a deficiency of 6.2 and $7.7 \mu \mathrm{m}$ PAH emission relative to $11.3 \mu \mathrm{m}$ PAH emission (Section 3.2.1), (2) morphological evidence for a radiative bow shock within an ionization cone aligned with radio plasma in an outflow (Section 3.2.4), (3) spectral properties of the luminous, hard X-ray point source (Section 3.2.5), and (4) a high $\mathrm{H}_{2}(1-0) \mathrm{S}(1)$ to $\mathrm{Br} \gamma$ flux ratio (Imanishi et al. 2009). Indeed, the strength and spatial extent of $\mathrm{H}_{2}$ emission indicate the presence of extensive shocks in the region (Section 3.2.2) that may be triggered by outflow from the AGN (Section 3.2.4).

\subsection{Between the Colliding Galaxies}

\subsubsection{A Search for Counterparts to the Radio Emission}

The bright radio continuum source located between the nuclei of Mrk 266 (Figures 8(a) and 19) was interpreted as a source of enhanced synchrotron emission induced by shocking of the ISM at the interface of the merging galaxies (Mazzarella et al. 1988). Direct imaging of this area with HST ACS and NICMOS, Spitzer IRAC and MIPS (Section 2.6.1), and spectral mapping with Spitzer IRS (Section 2.7) reveals no obvious source at optical continuum, near-IR, or mid-IR wavelengths. This strongly rules out any third (dust-obscured) galaxy nucleus or a luminous off-nuclear star-forming region as observed in other interacting systems such as the Antennae (Mirabel et al. 1998) and II $\mathrm{Zw} 96$ (Inami et al. 2010). Additionally, there is no detectable emission from this region in the near-UV or far-UV (Figures 7 and $8(\mathrm{~h})$ ), and there is no detection of warm molecular gas in the continuum-free $\mathrm{H}_{2} \mathrm{~S}(3) 9.67 \mu \mathrm{m}$ emission-line image (Figure 10). Although a bridge of CO (1-0) emission has been detected between the galaxies (Imanishi et al. 2009), it is shown below (Section 3.7) that this material is located to the north of the central radio continuum emission. Next we turn attention to the only other waveband where a source of radiation has been detected that is spatially correlated with the radio continuum emission between the galaxies.

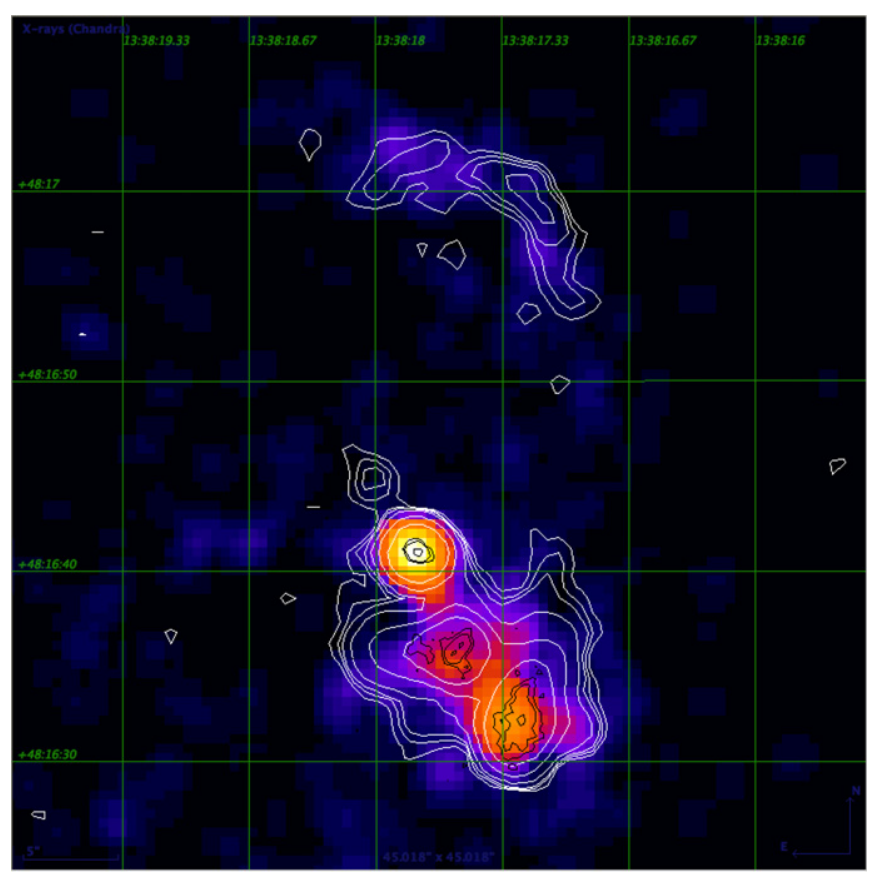

Figure 19. Mrk 266 Chandra $0.4-7 \mathrm{keV}$ (full band) X-ray image in pseudocolor, VLA $20 \mathrm{~cm}$ radio continuum in white contours, and VLA $6 \mathrm{~cm}$ radio continuum in black contours. The X-ray data are based on image processing described in Section 2.3.1 of this study and the radio data are from Mazzarella et al. (1988). The brightest $20 \mathrm{~cm}$ contours have been omitted so as not to obscure the $6 \mathrm{~cm}$ and X-ray emission (see also Figure 8). The field of view is $45^{\prime \prime} \times 45^{\prime \prime}$ and the coordinate grid is in $\mathrm{J} 2000$ equatorial coordinates.

(A color version of this figure is available in the online journal.)

\subsubsection{X-Rays Between the Nuclei}

Substantial soft X-ray emission has been detected between the nuclei of Mrk 266 by Chandra. Analysis by Brassington et al. (2007) indicates that the X-ray-emitting gas in this region has an intrinsic luminosity of $9.8( \pm 0.5) \times 10^{40} \mathrm{erg} \mathrm{s}^{-1}$ and has a temperature of $1.07 \mathrm{keV}$, approximately twice the temperature of much more diffuse emission surrounding the two galaxies (omitting the nuclei and northern source). Brassington et al. suggested that this X-ray emission is likely associated with the collision between the two galaxies, as proposed previously for the radio continuum emission (Mazzarella et al. 1988). The present work extends these results using reprocessing of the Chandra data to enhance the definition of structure in the diffuse X-ray emission and to further characterize its physical properties.

To begin with, Figures 3 and 19 illustrate the remarkable correspondence between the $0.4-7.0 \mathrm{keV}$ X-ray emission and the $20 \mathrm{~cm}$ radio continuum emission. As shown in Figures 8 and 19, the higher resolution $6 \mathrm{~cm}$ radio continuum emission $\left(0^{\prime} .3 \times 0\right.$.'.4 FWHM) resolves the $20 \mathrm{~cm}$ source into a flattened structure with two sub-components. These components are coincident with the brightest knot of soft (0.4-4.0 keV) X-ray emission located between the galaxies. The contours of the fullband (0.4-7.0 keV) X-ray image in Figure 18 further delineate the structure of the diffuse emission between the nuclei and to the southwest. Figure 18(b) presents the hardness of the soft $\mathrm{X}$-ray spectrum $\left(\mathrm{HR}_{\text {soft }}\right)$, which indicates temperature variations within the extended emission.

Figure 20 provides a detailed view of the enhanced soft $\mathrm{X}$-ray emission between the two galaxies. The X-ray spectra in the three circled areas show that the SE region has a softer spectrum, indicating a lower temperature $(k T \approx 0.35 \mathrm{keV})$ than 


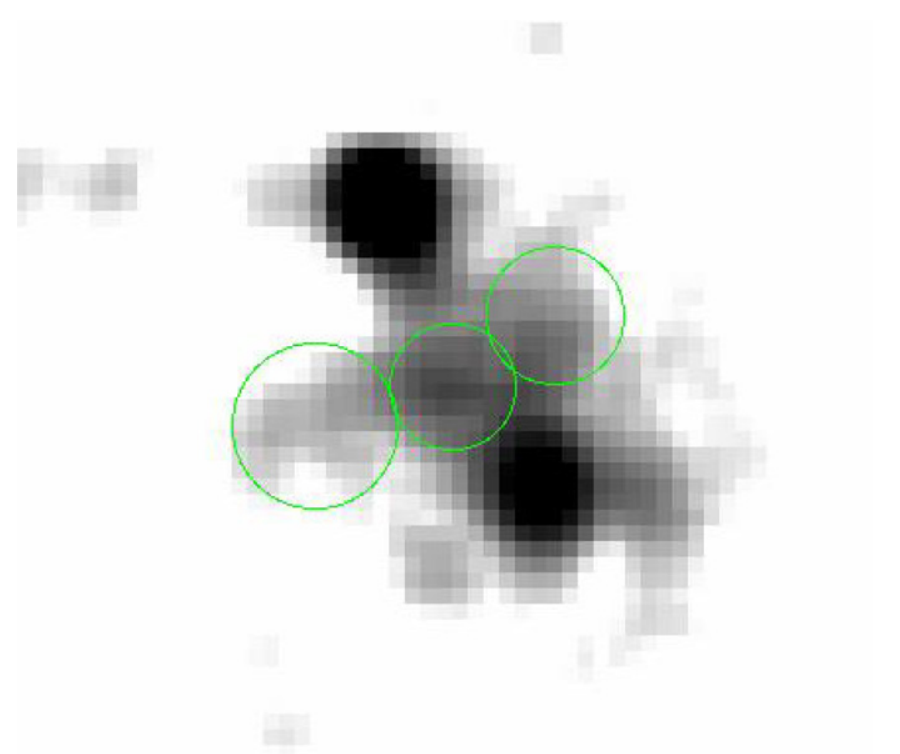

Figure 20. Close-up view of the Chandra soft-band (0.4-7 keV) X-ray emission in the region between the nuclei and to the SW. The three circled regions centered on the radio continuum peak (70 counts), $4^{\prime \prime}$ to the NW (70 counts), and $4^{\prime \prime} \mathrm{SE}$ (39 counts) are used to analyze the hardness of the X-ray emission to deduce differences in the gas temperature.

(A color version of this figure is available in the online journal.)

the other two regions $(k T \approx 0.8 \mathrm{keV})$. These differences are also reflected in the $\mathrm{HR}_{\text {soft }}$ map, where the $\mathrm{SE}$ region has $\mathrm{HR}_{\text {soft }}=-0.16$ and the region at the radio continuum peak and $4^{\prime \prime} \mathrm{NE}$ of that location have $\mathrm{HR}_{\text {soft }}$ ranging from 0.2 to 0.4 . Therefore, the X-ray brightness certainly increases at the radio peak, but the temperature of the $\mathrm{X}$-ray-emitting gas at that location is similar to the region $4^{\prime \prime}(2.4 \mathrm{kpc})$ to the NW. A caveat in this analysis is that when dust obscuration is also varying spatially, $\mathrm{HR}_{\mathrm{soft}}$ may not translate directly to real variations in temperature.

Table 7 presents physical parameters estimated from spectral analysis of the soft X-ray emission illustrated in Figure 20. The most straightforward interpretation is that the X-ray emission is the result of gas being shock heated to $T \sim 10^{7}$. This physical process would also give rise to the observed enhanced synchrotron radiation (Mazzarella et al. 1988). Davies et al. (2000) interpreted the lack of $\mathrm{H}_{2}$ 1-0 S(1) emission from the central region of Mrk 266 as evidence for very fast shocks with relative velocities of $\approx 1000 \mathrm{~km} \mathrm{~s}^{-1}$ produced at the interface of superwinds emerging from the two galaxies. Such fast shocks would generate the observed non-thermal radio continuum emission and also heat the gas to $\gtrsim 10^{6} \mathrm{~K}$, far above the dissociation temperature of $\mathrm{H}_{2}$. This would explain the lack of detected $\mathrm{H}_{2}$ emission lines between the galaxies in the nearinfrared observations of Davies et al. and in the mid-infrared IRS spectroscopy presented in the current study (Section 2.7). This physical scenario also predicts higher temperatures in the shocked region compared with the diffuse superwind. This is exactly what our refined analysis of the Chandra data reveals.

The estimated cooling time in this region is only 4 Myr. This is very short compared to the dynamical timescale of the merger (a few hundred million years), and therefore it is likely that we are observing this emission during a short-lived phase of the interaction. This would explain why so few interacting systems have been found with radio continuum and X-ray emission enhanced between the galaxies to the same extent as in Mrk 266. However, as shown below, there is evidence that gas in this
Table 7

Spectral Analysis of X-Rays Between the Nuclei

\begin{tabular}{lcc}
\hline \hline $\begin{array}{l}\text { Parameter } \\
(1)\end{array}$ & Value & $\begin{array}{c}\text { Units } \\
(3)\end{array}$ \\
\hline $0.5-2$ keV Flux & $(2)$ & $\mathrm{erg} \mathrm{s}^{-1} \mathrm{~cm}^{-2}$ \\
Temperature $(k T)$ & $2.7 \times 10^{-14}$ & $\mathrm{keV}^{+1}$ \\
Luminosity $^{\mathrm{a}}$ & $0.72_{-0.09}^{+0.11}$ & $\mathrm{erg} \mathrm{s}^{-1}$ \\
Volume $_{\text {Electron density }}$ & $1.4 \times 10^{41}$ & $\mathrm{~cm}^{3}$ \\
Thermal energy & $8.1 \times 10^{65}$ & $\mathrm{~cm}^{-3}$ \\
Gas mass & $1.1 \times 10^{-2}$ & $\mathrm{erg}^{5}$ \\
Cooling time & $1.8 \times 10^{55}$ & $M_{\odot}$ \\
\hline
\end{tabular}

Notes. Column 1: physical parameter computed from analysis of the soft band (0.5-2.0 keV) Chandra emission in the region between the colliding disks of Mrk 266. Column 2: value. Column 3: units. The computations assume an angular-scale distance of $129 \mathrm{Mpc}$ and the scale of $0.59 \mathrm{kpc} \operatorname{arcsec}^{-1}$ (see Section 1.3), and the emitting region is approximated by a tube $4^{\prime \prime}$ in diameter and $13^{\prime \prime}$ in length at a position angle of $-45^{\circ}$.

a The bolometric luminosity of this thermal gas, as deduced via extrapolation from fitting the data at energies of $0.01-10 \mathrm{keV}$.

region may be replenished from the powerful starburst-driven superwinds (Section 3.4) or from gravitational infall of material toward the center of mass of the system (Section 3.7).

\subsection{The Superwind and Northern Loop}

An outstanding characteristic of Mrk 266 is the extensive filamentary nebula of ionized hydrogen $\sim 30 \mathrm{kpc}$ in diameter (Armus et al. 1990) contained within an X-ray nebula $\sim 100 \mathrm{kpc}$ in extent. Optical emission-line diagnostics and kinematics indicate this is one of the most energetic examples of an outflowing, starburst-driven superwind (Wang et al. 1997). Mrk 266 has the third highest $\mathrm{H} \alpha$ luminosity in a sample of 40 local infrared-bright galaxies studied by Armus et al. (1990). Kollatschny \& Kowatsch (1998) presented deep observations with ROSAT HRI that revealed luminous soft X-ray emission spatially correlated with a loop of $\mathrm{H} \alpha$ and [O III] $\lambda 5007$ line emission $\sim 14^{\prime \prime}$ long by $7^{\prime \prime}$ wide extending $\sim 10^{\prime \prime}-34^{\prime \prime}(6-20 \mathrm{kpc})$ to the north of the system (herein called the Northern Loop), leading these authors to interpret the X-ray emission in this region as hot post-shock gas in a high-velocity "jet" (most likely emanating from the SW nucleus). Kollatschny \& Kowatsch (1998) also argued that the X-ray emission is too luminous and the opening angle of the $\mathrm{X}$-ray/[O III]-emitting region is too small to be explained by a superwind. A third possibility, suggested by Brassington et al. (2007), is that the northern structure is a tidal tail containing in situ star formation that is responsible for the soft X-ray emission and $\mathrm{H} \alpha$ emission. In the subsections below we exploit the new multiwavelength data to make the case that the Northern Loop is the most luminous component of a superwind energized by star formation and one or both of the AGNs.

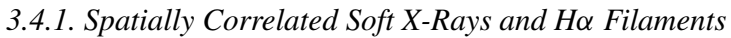

Our reprocessing of the Chandra data (Figure 21) reveals that the X-ray emission detected in the direct high-resolution data is a high surface brightness ridge embedded in a longer spur that extends an additional $\sim 20^{\prime \prime}(12 \mathrm{kpc})$ toward the NE. A map of the hardness of the soft X-ray spectrum (Figure 18) shows that the northern region has $\mathrm{HR}_{\text {soft }} \approx-0.2$, indicating a relatively cool temperature of $k T \approx 0.3 \mathrm{keV}$. If this is a region of in situ star formation, one would expect to find a 


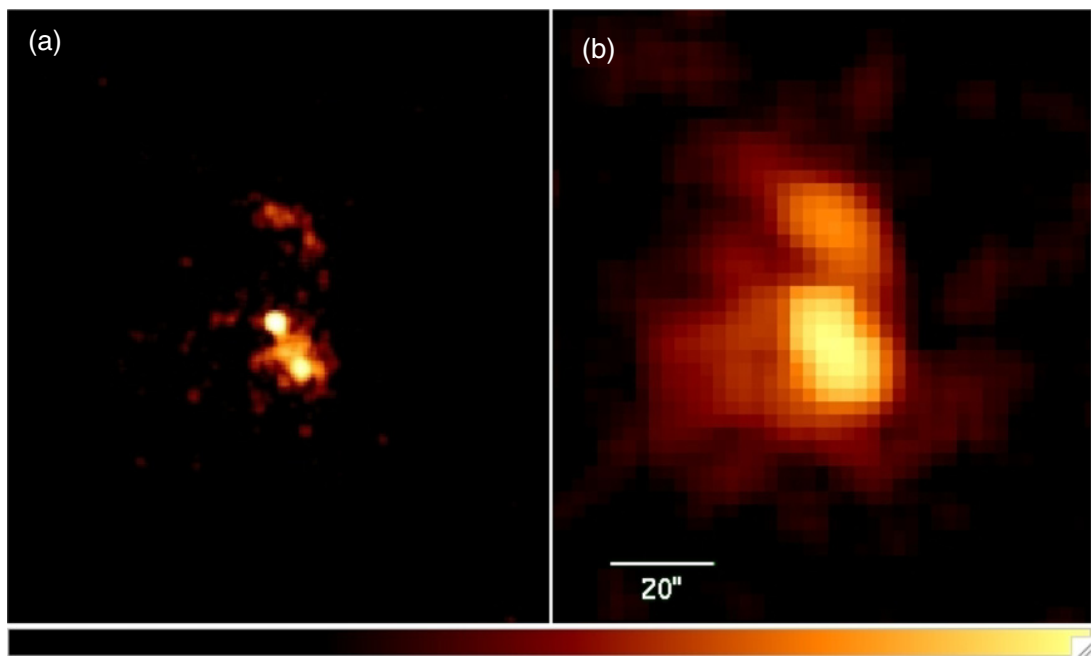

Figure 21. Chandra 0.4-7 keV (full band) X-ray data contrasting the high surface brightness emission (highest resolution) with the large-scale diffuse emission (a) processed for maximum spatial resolution and smoothed with a 2 pixel Gaussian kernel (same as Figure 3, bottom left) and (b) the same data smoothed with a 6 pixel Gaussian kernel to highlight diffuse emission.

(A color version of this figure is available in the online journal.)

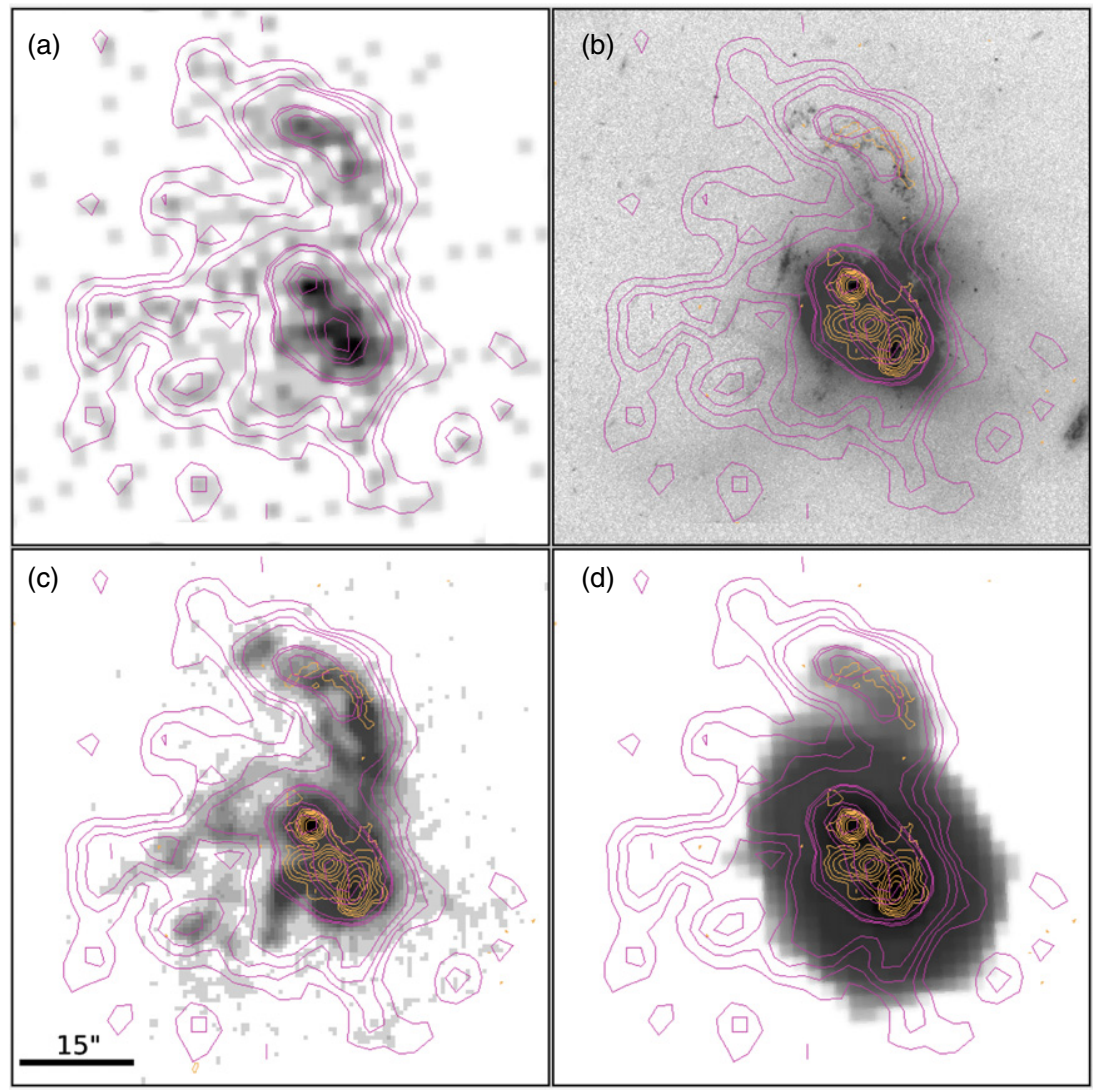

Figure 22. Soft X-rays compared to other wavelengths: (a) Chandra soft $(0.4-1 \mathrm{keV}) \mathrm{X}$-ray image re-binned with $2^{\prime \prime} \times 2^{\prime \prime}$ pixels (grayscale). The contours are the same data smoothed with a 3 pixel Gaussian kernel to emphasize the faint, diffuse emission; the same contours are also overlaid in the other three panels. (b) HST/ACS $B$-band $(0.44 \mu \mathrm{m}, \mathrm{F} 435 \mathrm{~W})$ image (grayscale) with the $0.4-1 \mathrm{keV} \mathrm{X}$-ray data overlaid as magenta contours and the VLA $20 \mathrm{~cm}$ radio continuum image (Mazzarella et al. 1988) overlaid as orange contours. (c) $\mathrm{H} \alpha$ image (Ishigaki et al. 2000) with the same contours as panel (b). (d) $24 \mu \mathrm{m}$ Spitzer MIPS image with the same contours as panels (b) and (c). Each field is 1'.2 $\times 1$.'2 and the scale bar indicates $15^{\prime \prime}$.

(A color version of this figure is available in the online journal.)

high density of SCs; however, the nearest detected optical SCs are more than $5^{\prime \prime}(3 \mathrm{kpc}) \mathrm{SW}$ from the brightest X-ray and radio continuum emission in the Northern Loop (Section 3.6). Therefore, the properties of this feature must be ascribed to something other than starlight. Figure 22 shows that the diffuse soft X-ray emission (0.4-1.0 keV) corresponds remarkably well with observed $\mathrm{H} \alpha$ filaments and knots that extend out to $\sim 18 \mathrm{kpc}$ $\left(30^{\prime \prime}\right)$ to the north and to the east of the galaxies. In the northern region, the soft $\mathrm{X}$-rays have the highest surface brightness near the outer edge of the Northern Loop detected in $\mathrm{H} \alpha$ and in 


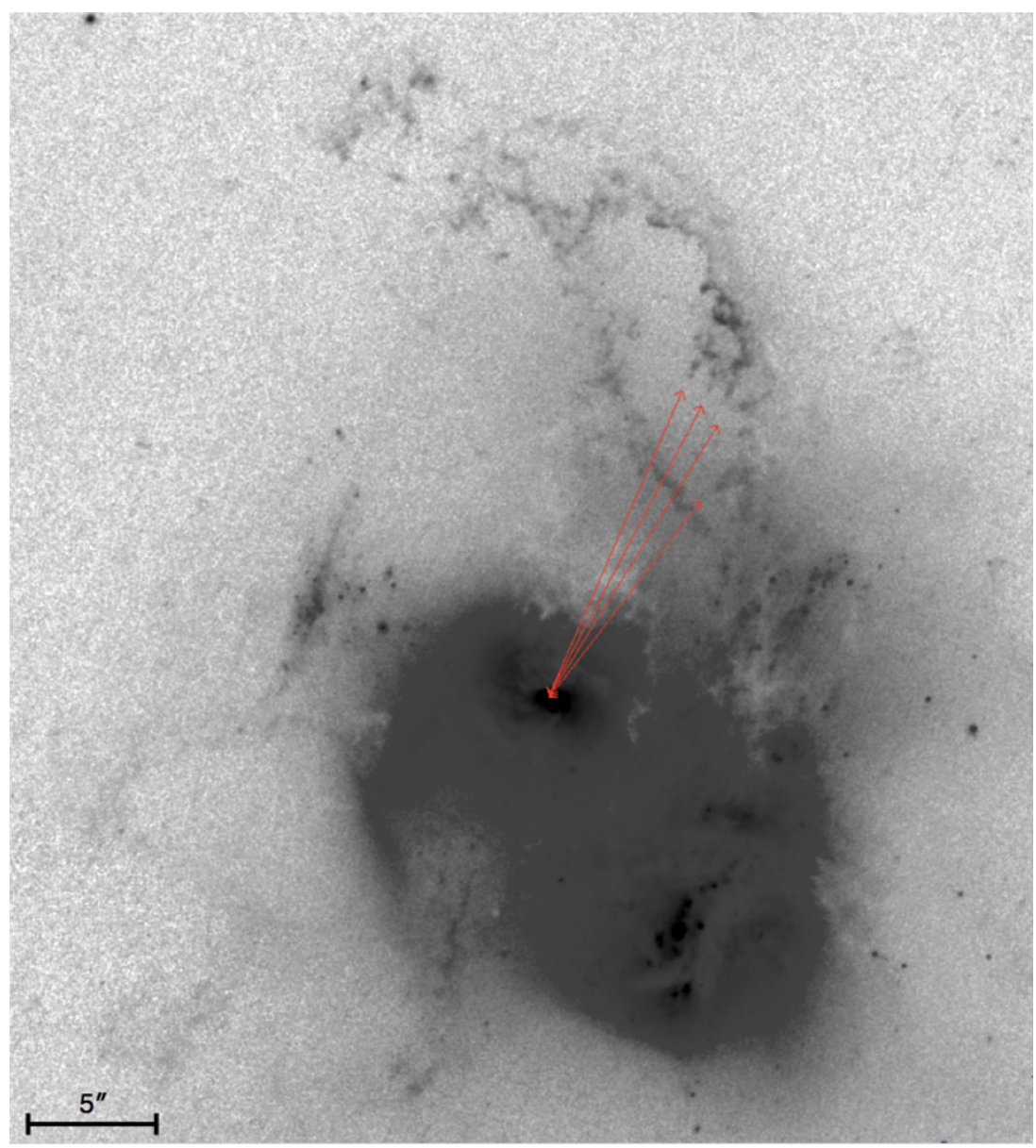

Figure 23. $H S T$ /ACS $B$-band $(0.44 \mu \mathrm{m}, \mathrm{F} 435 \mathrm{~W})$ image in grayscale with a field of view and intensity mapping chosen to emphasize the filamentary structure in the Northern Loop of Mrk 266. The arrows point out elongated structures $00^{\prime \prime} 5-1^{\prime \prime}(300-600 \mathrm{pc}$ ) in length on the western side of the loop that appear to be aligned radially with the NE nucleus.

(A color version of this figure is available in the online journal.)

the $B$ band (including [O II] 3727 line emission), which is also cospatial with $20 \mathrm{~cm}$ radio continuum emission. Close spatial correlation between hot X-ray-emitting gas and warm $\mathrm{H} \alpha$-emitting gas is a signature of winds in objects ranging from ULIRGs to dwarf starburst galaxies (Grimes et al. 2005).

\subsubsection{Structure in the Northern Loop at $\sim 100$ pc Scales}

The filamentary morphology of the Northern Loop revealed by the HST imagery is evidence for an expending shell or "bubble" that is fragmenting due to Rayleigh-Taylor instabilities in a starburst- or AGN-driven superwind. Three-dimensional hydrodynamic simulations of galactic winds predict this phenomenon (Cooper et al. 2008). Elongated structures $\approx 0$ '.5-1" $(300-600 \mathrm{pc})$ in length protruding inward from the western, outer edge of the Northern Loop are highly suggestive of Rayleigh-Taylor fingers or bow shocks predicted by simulations (e.g., Heckman et al. 1990; Cooper et al. 2008). These protrusions appear to have a radial distribution centered on the NE nucleus (Figure 23), indicating this AGN may be a primary energizing source for this component of the superwind. Wang et al. (1997) and Ishigaki et al. (2000) have shown that filaments throughout Mrk 266, including the Northern Loop, have line ratios (e.g., [O III] $\lambda 5007 / \mathrm{H} \beta$ ) indicating photoionization from an AGN dominates over ionization from massive stars, and shocks that could explain the LINER-like nebular spectrum have insufficient energy to account for the luminosity of the extended nebula. However, the luminous starburst in the SW galaxy is also likely to be a major contributor to the overall energetics of the superwind.

The morphological, kinematic, and ionization properties of the prominent Northern Loop can be interpreted with the superwind model of Heckman et al. (1990). In this scenario, the starburst-driven gaseous outflow has inflated a "bubble" that is highly elongated along the direction of least resistance (maximum pressure gradient), and the closed loop of optical line emission indicates that the superwind in Mrk 266 is in a relatively young radiative cooling phase that occurs before the wind expands further during a "blow-out" phase. This again is consistent with the idea that we are observing a relatively short-lived and dynamic phenomenon.

\subsubsection{Dust in the Wind}

The detection of $24 \mu \mathrm{m}$ emission cospatial with the Northern Loop (Figure 22(d)) is strong evidence for thermal continuum emission from dust grains entrained in the superwind. By estimating the dust temperature and grain emissivity profile, the mass of dust can be derived following the single temperature thermal model of Hildebrand (1983), which is given by

$$
M_{\text {dust }}=\left[(4 / 3) a \rho / Q_{v}\right]\left[S_{v} D^{2} / B(T, v)\right],
$$

where $a$ is the average grain size $(0.1 \mu \mathrm{m}), \rho$ is the density of grain material, $Q_{v}$ is the grain emissivity characterized 
as $Q_{250 \mu \mathrm{m}}(250 \mu \mathrm{m} / \lambda)^{\beta}, S_{\nu}$ is the flux density of the thermal emission at frequency $v, D$ is the distance, and $B(T, v)$ is the Planck function. Assuming $\beta=1$ and other grain properties from Hildebrand (1983) results in [(4/3) $\left.a \rho / Q_{24 \mu \mathrm{m}}\right]=$ $9.6 \times 10^{-3} \mathrm{~g} \mathrm{~cm}^{-2}$ at $24 \mu \mathrm{m}$. In more convenient units, the above equation becomes

$$
M_{\text {dust }}=0.016 S_{v}(24 \mu \mathrm{m}) D^{2}\left[e^{\left(599.49 / T_{\text {dust }}\right)}-1\right] M_{\odot},
$$

where $S_{v}(24 \mu \mathrm{m})$ is in Jy, $D$ is in Mpc, $T$ is in $\mathrm{K}$, and $M_{\text {dust }}$ is in $M_{\odot}$. The $24 \mu \mathrm{m}$ flux density measured for the Northern Loop (17 mJy or $2 \%$ of the total flux) corresponds to a dust mass $M_{d}(24 \mu \mathrm{m}) \approx 5 \times 10^{7} M_{\odot}$. Using the calibration of Alton et al. (1999) based on the known case of M82, the total far-infrared luminosity ratio of $L_{\mathrm{ir}}(\mathrm{Mrk} 266) / L_{\mathrm{ir}}(\mathrm{M} 82)=18$ predicts a dust mass of $\sim 2 \times\left(10^{7}-10^{8}\right) M_{\odot}$ in the entire wind of Mrk 266. This would imply that the $24 \mu \mathrm{m}$ emission from the Northern Loop contains at least $25 \%$ (and possibly the majority) of the total dust mass within the superwind of Mrk 266.

However, this result depends on the assumption that most of the observed flux is due to dust emission alone and that we have made a reasonable determination of $T_{\text {dust }}$. One possible source of alternate emission is the [O IV] $25.9 \mu \mathrm{m}$ emission line. We estimate this contribution to be less than $10 \%{ }^{30}$ Since there is no detection in any other mid-IR or submillimeter passband available to help constrain $T_{\text {dust }}$, a characteristic temperature of $37 \mathrm{~K}$ is assumed based on modeling of submillimeter observations of the dusty outflow in M82 (Alton et al. 1999).

The overall physical interpretation presented above adds strong support for the superwind interpretation of Wang et al. (1997) in which stellar winds and SNe are driving a powerful outflow of gas and dust. The Northern Loop is likely the cross section of the most luminous "bubble" of expanding ionized gas in the wind. The fragmented morphology and radial orientation of protrusions in this region suggest it is strongly influenced by radiation or a gaseous outflow energized by the NE galaxy, and the detection of cospatial $24 \mu \mathrm{m}$ emission provides strong evidence for dust mixed with the ionized gas in the superwind. The new observations are difficult to reconcile with a jet (ejection from an AGN) as suggested by Kollatschny \& Kowatsch (1998) or with a (stellar) tidal tail as hypothesized by Brassington et al. (2007).

\subsection{SED Analysis}

\subsubsection{Decomposition of Dust Emission Components}

Figure 24 presents results of a multicomponent model decomposition of the IRS spectrum (Figure 11) combined with the broadband SED (Table 12 and Figure 9) of global measurements for the Mrk 266 system using the CAFE software package (Marshall et al. 2007). ${ }^{31}$ A very good fit to the SED is provided by a model consisting of PAH emission, atomic and molecular line emission, plus cold (26 K), cool (72 K), warm (235 K), and hot $(1500 \mathrm{~K})$ thermal dust components heated by the combination of an unobscured photospheric interstellar radiation field

\footnotetext{
30 This estimate is based on the assumption that the mixture of ionized gas and thermal dust in the superwind of Mrk 266 may resemble a scaled up version of a planetary nebula such as NGC 2346 (Su et al. 2004). The published IRS spectrum of Mrk 266 shows a bright [O IV] $25.9 \mu \mathrm{m}$ emission line, however it does not permit separation of emission from the SW nucleus and the diffuse emission in the Northern Loop.

31 CAFE could not be used to constrain the fits for the individual galaxies because the IRS spectral map could not resolve the galaxies over the 14-38 $\mu \mathrm{m}$ range of the LL module (Section 2.7).
}

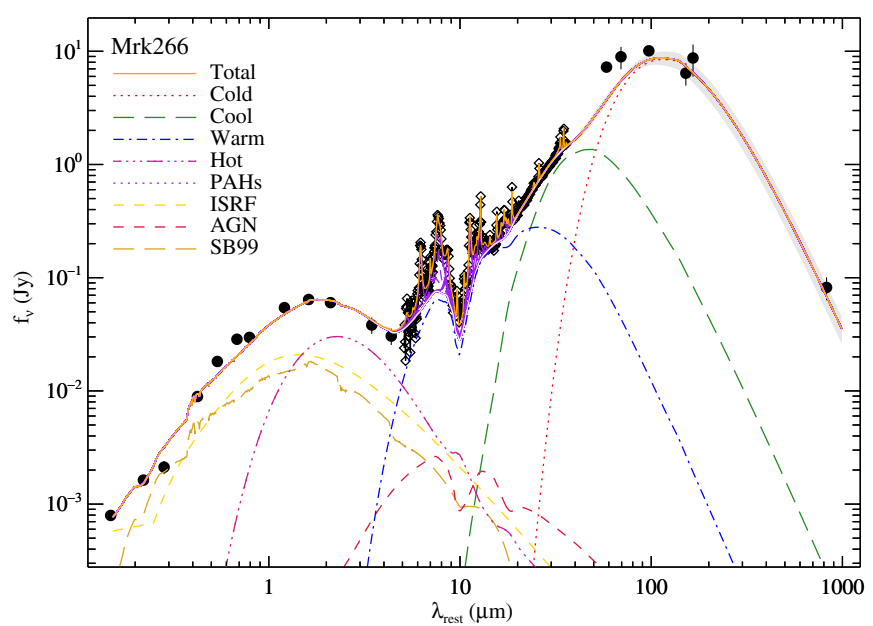

Figure 24. Results of multicomponent fitting using the CAFE software package (Marshall et al. 2007) to model the SED of the global emission from Mrk 266. Solid black circles are broadband continuum measurements, and open black squares are IRS spectral measurements. The solid orange curve represents the sum of the various fitted components identified in the legend. The corresponding fit parameters and uncertainties are given in Table 8.

(A color version of this figure is available in the online journal.)

(ISRF), an obscured starburst (SB) component with a strength constrained by the observed PAH emission, and an AGN (accretion disk) component with a strength constrained by the fitted hot dust emission. Uncertainties in the derived dust temperatures are $\pm 1 \mathrm{~K}$.

Table 8 lists the results of the fit. The derived extinctioncorrected PAH flux ratios appear to be fairly "normal," located between the curves for ionized and neutral PAHs in Figure 16 of Draine \& Li (2001). The parameter $L_{\text {source }}$ is the sum of the derived intrinsic source luminosities $L_{\mathrm{ISRF}}+L_{\mathrm{SB}}+L_{\mathrm{AGN}}$, and $L_{\text {dust }}$ is the total dust luminosity. The primary results are the relative fractions of $L_{\text {source }}$, the fractions of $L_{\text {dust }}$, and the dust masses characterized as arising from cold, cool, warm, and hot thermal dust components. As noted in Marshall et al. (2007), the four dust components used to model galaxies are typically heated to characteristic temperatures of $T_{\text {cold }} \approx 35 \mathrm{~K}, T_{\text {cool }} \approx$ $80 \mathrm{~K}, T_{\text {warm }} \approx 200 \mathrm{~K}$, and $T_{\text {hot }} \approx 1400 \mathrm{~K}$, where the actual temperatures (noted above and in Table 8 for Mrk 266) are determined from fitting of the radiation field energy density of the corresponding radiation sources. Although the model suggests that a hot dust component accounts for $\approx 10 \%$ of the total dust luminosity, this is uncertain because the fitting of this component is weighted heavily by the mid-IR wavelength region where emission from hot dust competes with stellar photospheric emission. Reconciliation of this relatively low AGN contribution with spectral diagnostics that suggest a $50 \%$ AGN contribution in the individual galaxies (Section 3.2.1) may be due primarily to an aperture effect. The latter measurements were made in apertures that isolate the nuclear regions, and they are thus more sensitive to AGN signatures than the larger apertures used to construct the SED for the global system which encompass more spatially extended starburst emission.

It is illustrative to compare Mrk 266 with the galaxies modeled by Marshall et al. (2007). Overall, the SED of Mrk 266 can be modeled as a starburst-dominated LIRG, with weak AGN indicators from the thermal dust or PAH emission, as is also the case in NGC 6240 and NGC 2623. While the cold dust component in Mrk 266 has essentially the same temperature as derived for NGC 6240 (29 K), the cool (72 K) and warm 
Table 8

Results of SED Decomposition

\begin{tabular}{ll}
\hline \hline $\begin{array}{l}\text { Parameter } \\
(1)\end{array}$ & \multicolumn{1}{c}{$(2)$} \\
\hline$\tau_{\text {warm }}(9.7 \mu \mathrm{m})^{\mathrm{a}}$ & $2.7 \pm 0.1$ \\
$P A H 6.2 \mu \mathrm{m} / \mathrm{PAH} 7.7 \mu \mathrm{m}$ & $0.28 \pm 0.03$ \\
$P A H 11.3 \mu \mathrm{m} / \mathrm{PAH} 7.7 \mu \mathrm{m}$ & $0.28 \pm 0.02$ \\
$L_{\mathrm{ISRF}} / L_{\text {source }}$ & 0.19 \\
$L_{\mathrm{SB}} / L_{\text {source }}$ & 0.71 \\
$L_{\mathrm{AGN}} / L_{\text {source }}$ & 0.10 \\
$L_{\text {source }}\left(L_{\odot}\right)$ & $2.4( \pm 0.2) \times 10^{11}$ \\
$L_{\text {cold }}(26 \mathrm{~K}) / L_{\text {dust }}$ & $0.57 \pm 0.09$ \\
$L_{\text {cool }}(72 \mathrm{~K}) / L_{\text {dust }}$ & $0.20 \pm 0.03$ \\
$L_{\text {warm }}(235 \mathrm{~K}) / L_{\text {dust }} \mathrm{b}$ & $0.12 \pm 0.01$ \\
$L_{\text {hot }}(1500 \mathrm{~K}) / L_{\text {dust }}$ & $0.11 \pm 0.01$ \\
$L_{\text {dust }}\left(L_{\odot}\right)$ & $2.2( \pm 0.2) \times 10^{11}$ \\
$M_{\text {cold }}(26 \mathrm{~K})\left(M_{\odot}\right)$ & $1.5( \pm 0.4) \times 10^{8}$ \\
$M_{\text {cool }}(72 \mathrm{~K})\left(M_{\odot}\right)$ & $1.5( \pm 0.6) \times 10^{5}$ \\
$M_{\text {warm }}(235 \mathrm{~K})\left(M_{\odot}\right)$ & $7.0( \pm 0.2) \times 10^{2}$ \\
$M_{\text {hot }}(1500 \mathrm{~K})\left(M_{\odot}\right)$ & 6.0 \\
\hline
\end{tabular}

Notes. Parameters resulting from SED decomposition using the sum of ISRF + starburst + AGN radiation sources, as described in the text and plotted in Figure 24.

a These values are likely closer to the intrinsic physical attenuation than the much lower value of $\tau_{\text {warm }}(9.7 \mu \mathrm{m})=0.7 \pm 0.1 \mathrm{de}$ rived from spline fitting that estimates only the apparent strength of the silicate absorption feature (Section 3.2.1).

b The $L_{\text {warm }} / L_{\text {dust }}$ values are corrected for the attenuation by $1-\mathrm{e}^{-\tau_{\mathrm{warm}}(9.7 \mu \mathrm{m})} / \tau_{\mathrm{warm}}(9.7 \mu \mathrm{m})$.

(235 K) dust components of Mrk 266 are significantly warmer than those in NGC 6240 ( $61 \mathrm{~K}$ and $193 \mathrm{~K}$ ). Together the warm and hot components comprise $23 \%$ of the total dust luminosity of Mrk 266. For context, the sum of the (apparent) warm and hot dust fractions are 22\% in NGC 6240, 77\% in Mrk 463, and 82\% in the QSO PG 0804+761. Within the uncertainties, Mrk 266 has the same cold dust mass as NGC 6240. Compared to the other two dual-AGN systems which have had SED decomposition using this technique, the $T_{\text {dust }}$ distribution in Mrk 266 is quite similar to NGC 6240, and both of these systems have much smaller fractions of warm+hot dust than Mrk 463. The Mrk 266 SED fitting results also illustrate that only $700 M_{\odot}$ of dust at $T \gtrsim 200 \mathrm{~K}$ can generate the same luminosity $\left(4.4 \times 10^{10} L_{\odot}\right)$ as $1.5 \times 10^{5} M_{\odot}$ of dust at $T \approx 72 \mathrm{~K}$.

\subsubsection{Flux Ratios}

The physical nature of AGNs is often revealed via flux ratios. We begin by determining the " $q$ " parameter as defined by Yun et al. (2001):

$q_{\mathrm{FIR}}=\log \left(\frac{\mathrm{FIR}}{3.75 \times 10^{12}\left[\mathrm{~W} \mathrm{~m}^{-2}\right]}\right)-\log \left(\frac{S_{1.4 \mathrm{GHz}}}{\left[\mathrm{W} \mathrm{m}^{-2} \mathrm{~Hz}^{-1}\right]}\right)$,

where FIR $\left[\mathrm{W} \mathrm{m} \mathrm{m}^{-2}\right]=1.26 \times 10^{-14}\left(2.58 \cdot \mathrm{S}_{60 \mu \mathrm{m}}[\mathrm{Jy}]+\right.$ $\left.\mathrm{S}_{100 \mu \mathrm{m}}[\mathrm{Jy}]\right)$, and the flux densities are those measured by IRAS for the global system. In addition, the $q_{\text {FIR }}$ parameter can be defined as

$$
q_{24}=\log \left[\left(S_{24 \mu \mathrm{m}}\right) /\left(S_{1.4 \mathrm{GHz}}\right)\right],
$$

where $q_{24}$ is the $24 \mu \mathrm{m}$ flux density measured by Spitzer MIPS. The data in Tables 12 and 13 give $q_{\text {FIR }}=2.0$ and $q_{24}=0.95$ for the global system, and $q_{24}=1.77$ (1.14) for Mrk $266 \mathrm{SW}$ (NE). Mrk 266 is typical of galaxies in the IRAS 2 Jansky Survey, 98\% of which fall in the range $1.64 \leqslant\left\langle q_{\text {FIR }}\right\rangle \leqslant 3.02$ (population mean $\left.\left\langle q_{\mathrm{FIR}}\right\rangle=2.34\right)$. A useful comparison for the $q_{24}$ values is the sample mean $\left\langle q_{\text {FIR }}\right\rangle=0.83 \pm 0.31$ ( $1 \sigma$ dispersion) for over 770 galaxies in the Spitzer First Look Survey (FLS) with redshifts less than 0.4 (Marleau et al. 2007). The global system and Mrk $266 \mathrm{NE}$ are within $1 \sigma$ of this sample mean, and although $q_{24}$ for Mrk $266 \mathrm{SW}$ is large compared to most LIRGs, it is within the $3 \sigma$ dispersion of the FLS population. Therefore, Mrk 266 shows no significant deviations from the infrared-radio correlation followed by star-forming galaxies and radio-quiet AGNs.

The ratio of infrared to ultraviolet luminosities provides a diagnostic of the amount of reprocessed versus escaping radiation from young, massive stars. Mrk 266 has an observed ratio of $L_{\mathrm{ir}} / L_{\mathrm{UV}}=20$, where $L_{\mathrm{UV}}$ is the sum of the NUV and FUV luminosities measured by GALEX, and it has a ratio $L_{\mathrm{UV}} /\left(L_{\mathrm{ir}}+L_{\mathrm{UV}}\right)=0.047(5 \%)$. With a correction for the finding that $L_{\mathrm{ir}}$ is powered roughly equally by the AGNs and star formation (Section 3.2.1), about $10 \%$ of the total star formation in Mrk 266 is observable directly as escaping UV photons, and $90 \%$ is UV radiation reprocessed into thermal infrared emission. Since the SFR scales directly with the starburst component of $L_{\mathrm{UV}}+L_{\mathrm{ir}}$, the system has $\mathrm{SFR}_{\mathrm{UV}} / \mathrm{SFR}_{\mathrm{Total}}=10 \%$, which is on the high end of the distribution for the GOALS sample ranging $0.2 \%-18 \%$ with mean $3 \%$ (Howell et al. 2010).

Lastly we examine the ratio of observed hard X-ray luminosity (uncorrected for obsorption) to infrared luminosity. Among 13 local ULIRGs studied by Armus et al. (2007), those with AGN-dominated mid-IR spectra have a ratio of hard X-rays to infrared luminosity of $L_{h x} / L_{\mathrm{ir}} \gtrsim 10^{-3}$, whereas starburst-dominated ULIRGs have $L_{h x} / L_{\mathrm{ir}}<10^{-4}$. The XMMNewton measurement for the global Mrk 266 system gives $L_{h x} / L_{\mathrm{ir}}=3.5 \times 10^{-4}$, and the higher resolution Chandra measurements give $L_{h x} / L_{\mathrm{ir}}=5.0 \times 10^{-5}$ and $L_{h x} / L_{\mathrm{ir}}=1.0 \times 10^{-3}$ for the SW and NE nuclei, respectively. The component galaxies of Mrk 266 are therefore at extreme ends of the range observed to date in the local (U)LIRG population. The value of $L_{h x} / L_{\text {ir }}$ for Mrk $266 \mathrm{NE}$ is 40\% larger than NGC 6240, which itself is similar to ratios found in optical/UV-selected Seyfert galaxies and quasars (Ptak et al. 2003). Mrk 266 SW, on the other hand, has one of the smallest $L_{h x} / L_{\text {ir }}$ ratios among local (U)LIRGs, similar to Arp 220 and IRAS 22491-1808 (Armus et al. 2007). This is consistent with new evidence presented in Section 3.2.5 that the X-ray source in Mrk $266 \mathrm{SW}$ is Compton thick. The fact that the luminosity ratio of the SW and NE galaxies in hard X-rays $\left(L_{h x}(\mathrm{SW}) / L_{h x}(\mathrm{NE})=0.16\right)$ is close to the reciprocal of the ratio in the infrared $\left(L_{\mathrm{ir}}(\mathrm{SW}) / L_{\mathrm{ir}}(\mathrm{NE})=\right.$ $3.3)$ and in cold molecular gas $\left(L_{\mathrm{CO}}(\mathrm{SW}) / L_{\mathrm{CO}}(\mathrm{NE})=4.9\right.$; Section 3.7 ) is a strong clue that the factor of $\approx 20$ disparity in the $L_{h x} / L_{\text {ir }}$ ratios for these galaxies is mainly due to differences in the column density of material absorbing UV/X-ray photons and re-emitting the energy in the far-infrared, rather than an order of magnitude difference in the intrinsic hard X-ray luminosities of the AGNs.

This same conclusion about high column density material as the responsible agent for the variance of flux ratios can be arrived at by simply comparing the two nuclei. The data presented in Section 2.6.2 show that the luminosity ratio of the two galaxies, $L($ Mrk $266 \mathrm{SW}) / \mathrm{L}($ Mrk $266 \mathrm{NE})$, is $\approx 0.7$ in the far-UV and soft X-ray bands, transitions through 1.0 in the near-infrared $(1.2 \mu \mathrm{m})$, increases to a maximum of 5.7 at $8 \mu \mathrm{m}$, and drops to 3.5 at $70 \mu \mathrm{m}$ (the SED peak) and 3.3 in the total infrared emission $\left(L_{\mathrm{ir}}\right)$. The $L(\operatorname{Mrk} 266 \mathrm{SW}) / \mathrm{L}(\mathrm{Mrk} 266 \mathrm{NE})$ ratio drops further to 2.3 at $850 \mu \mathrm{m}$ and to 1.4 at $20 \mathrm{~cm}$. Thus, although 


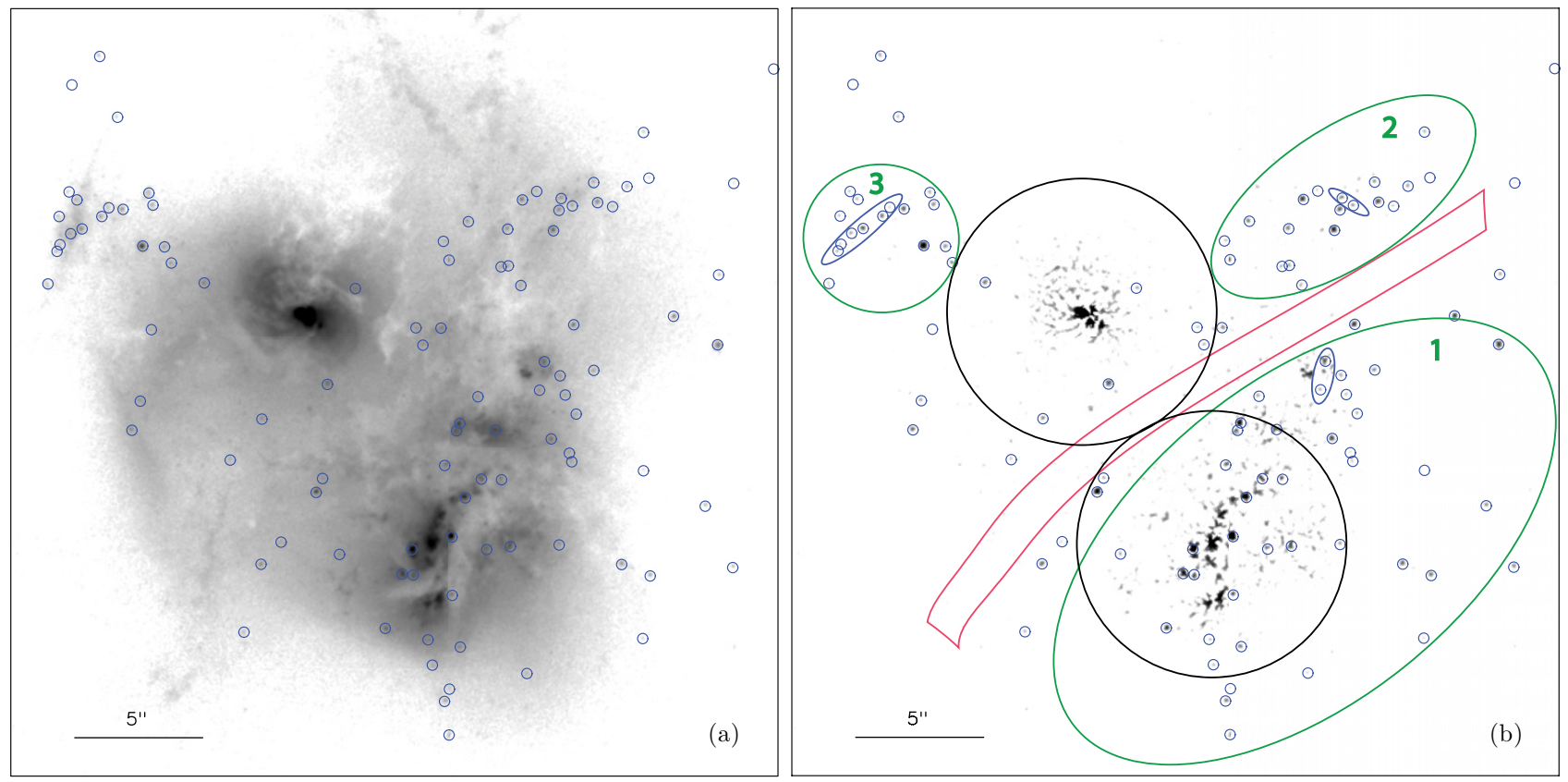

Figure 25. Star clusters (SCs) extracted by Source Extractor identified with small circles overlaid on the $0.44 \mu \mathrm{m}$ (B, F435W) HST image (a) before background subtraction and (b) after background subtraction using a median-masking technique. Panel (b) also identifies notable regions discussed in the text: three areas where most of the SCs are concentrated (large green ellipses), each of which contains a smaller concentration of very blue SCs (blue ellipses; see also Figure 26); regions with radius $5^{\prime \prime}$ centered on the nuclei of the two galaxies (black circles); and a dearth of SCs bisecting the system at P.A. $\approx-47^{\circ}$ (red band).

(A color version of this figure is available in the online journal.)

Mrk 266 SW clearly dominates this merger in the mid-IR and far-IR, the two galaxies have similar luminosities in the near-IR, $\mathrm{UV}$, soft X-rays, and radio continuum. It is only at the highest energies with available data for the separate galaxies where the situation is reversed: Mrk $266 \mathrm{NE}$ is 6.4 times more luminous in the hard (2-7 keV) X-ray band than its companion Mrk 266 SW. As shown in Section 3.2.5, this is more likely due to a higher column density of absorbing material along our line of sight to the SW nucleus (compared to the NE nucleus) than to an intrinsic difference in their power sources.

\subsection{Star Clusters}

\subsubsection{Cluster Detection and (B - I) Color Map}

The HST/ACS images of Mrk 266 reveal numerous very compact, marginally resolved sources scattered throughout the system. Their overall properties indicate they are SCs and associations similar to those observed in other galaxies undergoing extensive bursts of star formation. The detection method followed the procedure described in detail by Modica et al. (2012) for SCs in the late stage LIRG merger IC 883. First, masks outlining the galaxy were applied to the images. Then Source Extractor (Bertin \& Arnouts 1996) was used to fit and subtract the underlying galaxy and perform point-source detection in the $B$ - and $I$-band images. The extracted objects were processed with IDL routines to perform photometry and apply selection criteria that accepted only reliable sources with an FWHM of 1.7-4 pixels, have $\mathrm{S} / \mathrm{N}>5$, and are detected in both bands; details are given in Modica et al. (2012). This procedure resulted in the detection of 120 point sources with $21 \mathrm{mag} \lesssim B \lesssim 27 \mathrm{mag}$ and $19.5 \mathrm{mag} \lesssim I \lesssim 25.7 \mathrm{mag}$ (uncorrected for extinction); their coordinates and photometric properties are listed in the Appendix (Table 15).

Figure 25 shows the direct $H S T$ B-band image (a) and the image after processing with Source Extractor to fit and subtract the local background and extract SCs (b). The density of SCs is highest in three extended regions identified with green ellipses in Figure 25(b): (1) within and surrounding the disk of the SW galaxy, (2) along a band extending $\sim 5^{\prime \prime}-15^{\prime \prime}(3-9 \mathrm{kpc}$ ) northwest from the NE galaxy, passing near the base of the Northern Loop, and (3) in a group centered $\sim 8^{\prime \prime}(5 \mathrm{kpc})$ northeast of the NE nucleus. There are $22 \mathrm{SCs}$ within a projected radius of $5^{\prime \prime}$ (3 kpc) around the nucleus of Mrk 266 SW, and only 6 SCs within the same radius around Mrk $266 \mathrm{NE}$ (black circles in Figure 25(b)). The ratio of surface densities of detected clusters in the SW and NE galaxies (3.7) is nearly the same as the ratio of their infrared luminosities (3.5, see Section 3.5), and it is also similar to the ratio of their cold molecular gas masses (4.9, see Section 3.7). This situation is consistent with the apparent Hubble types and $1.6 \mu \mathrm{m}$ bulge-to-disk ratios (Section 3.1.1) of the two galaxies (i.e., SBb disk galaxies contain more gas to fuel star formation than S0/a galaxies). Despite a nearly four to one ratio in the number of clusters detected in the central $3 \mathrm{kpc}$ of the SW and NE galaxies, across the entire system there is a nearly equal number of clusters on both sides of a notable gap about $2^{\prime \prime}(1.2 \mathrm{kpc})$ wide running between the galaxies at P.A. $\approx-47^{\circ}$ (red band in Figure 25(b)).

A color map revealing the variation of $(B-I)$ magnitudes throughout Mrk 266 is shown in Figure 26, with an overlay of symbols coded to indicate the $(B-I)$ colors of the SCs (uncorrected for extinction). Dust lanes present in the direct images (Figures 1, 13, and 14) are more clearly delineated in this color map as filamentary regions with $(B-I) \gtrsim 2$ mag. In the SW galaxy, the reddest dust lanes are on the east and west sides of the nucleus, and aligned with the major axis of the underlying starlight detected in the $1.6 \mu \mathrm{m}$ image (Figure 3); they are also aligned with the apparent rotation axis of the molecular gas (see Section 3.7). In the NE galaxy, the dust lane has an "S" shape that crosses the nucleus at P.A. $\approx-10^{\circ}$. Higher than average extinction explains the lack of detected clusters in these 


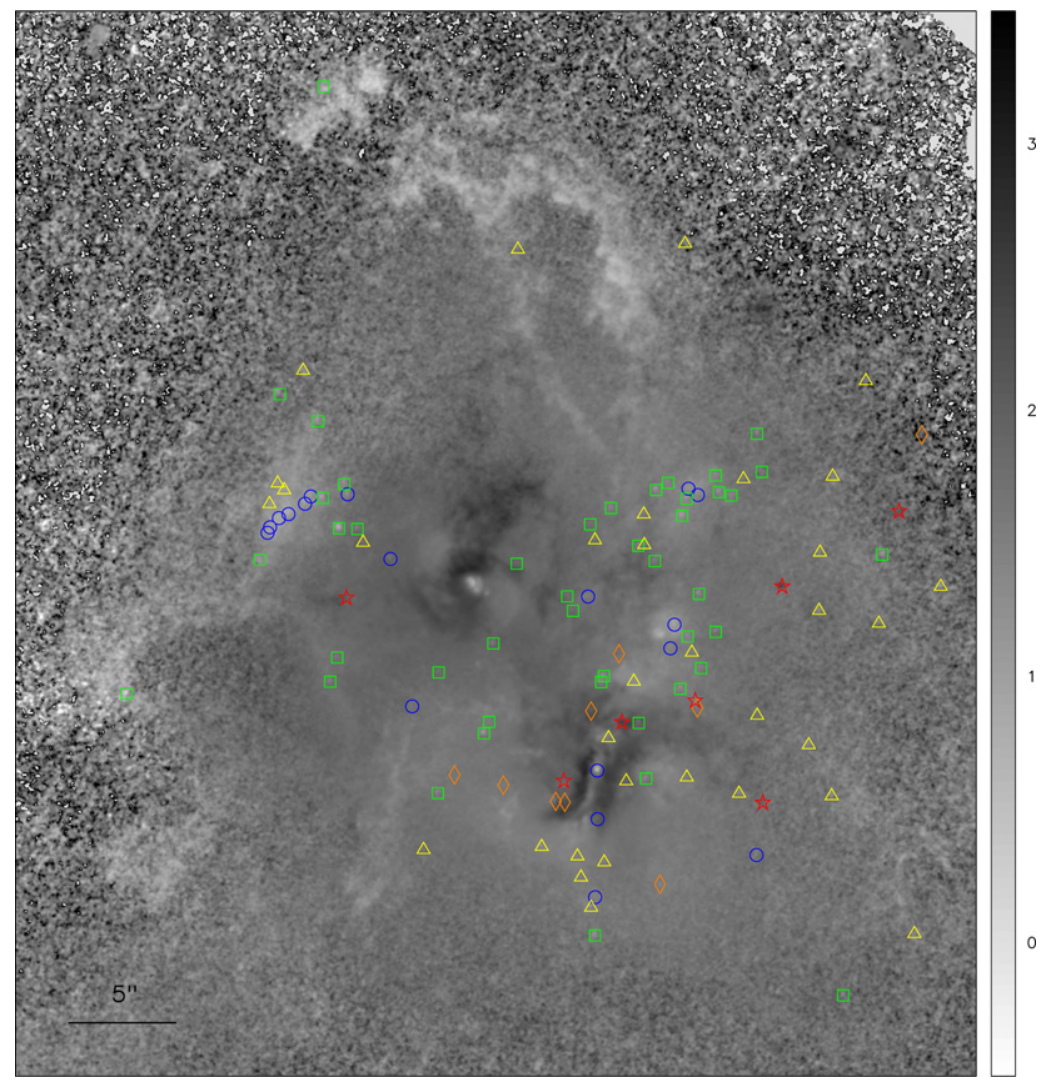

Figure 26. Gray-scale F435W-F814W $(B-I)$ color map of Mrk 266, with star cluster positions color-coded by their individual $(B-I)$ colors in ranges as follows: [F435W]-[F814W] $(B-I)=0-0.5$ mag: blue circles; 0.5-1 mag: green squares; $1-1.5$ mag: yellow triangles; $1.5-2.0$ mag: orange diamonds; $(B-I)>2.0$ mag: red stars. The scale bar on the right indicates the $(B-I)$ colors of the pixels as observed, uncorrected for extinction (reddening).

(A color version of this figure is available in the online journal.)

circumnuclear dust lanes. However, in the region between the galaxies the color map shows bluer $(B-I)$ values, suggesting the apparent dearth of clusters along this gap (red band in Figure 25(b)) may be intrinsic.

Some of the bluest SCs (blue circles in Figure 26) are located in regions identified with blue ellipses in Figure 25(b), including groupings centered $\sim 7^{\prime \prime}$ and $\sim 13^{\prime \prime}$ northwest of the SW nucleus (inside regions 1 and 2 noted above), and a notable chain $\approx 5^{\prime \prime}$ in length located northeast of the NE nucleus (inside region 3 noted above). The detected clusters are a direct probe of star formation occurring in regions with relatively low extinction. However, the fact that the total luminosity of the unobscured clusters $\left(M_{B}=-16.25 \mathrm{mag}\right.$ or $\left.L_{B}=5 \times 10^{8} L_{\odot}\right)$ is only $0.1 \%$ $(1.6 \%)$ of the total infrared ( $B$-band) luminosity indicates that these clusters provide a minor contribution to the total energy output of the system.

The very blue colors of the Northern Loop and filaments throughout the system are likely a signature of strong nebular [O II] $\lambda 3727 \AA$ and [O III] $\lambda 4364 \AA$ line emission in the $B$ band (see Section 3.4). The NE nucleus, the arc 0.'4 to the west, and the bi-conic structure in which they are embedded are comparably blue, with $(B-I) \approx-0.2 \mathrm{mag}$. Similarity in color with filaments throughout the system that are visible in [O III] emission-line imaging (Ishigaki et al. 2000) lends support to the hypothesis presented in Section 3.2.4 that the circumnuclear region of Mrk $266 \mathrm{NE}$ is an AGN ionization cone.

\subsubsection{Star Cluster Color-Magnitude Diagram}

Figure 27 is a $B-I$ versus $M_{B}$ color-magnitude diagram with evolutionary tracks constructed using population synthesis models from Bruzual \& Charlot (2003). Most of the SCs have observed (apparent) colors of $0.5 \mathrm{mag} \leqslant(B-I) \leqslant 2.0 \mathrm{mag}$ and $-9 \mathrm{mag} \leqslant M_{B} \leqslant-13 \mathrm{mag}$ (Figure 27(a)). Although there is an age/mass degeneracy in the uncorrected measurements, at first glance most of the SCs appear to have ages of a few hundred Myr to 1 Gyr. However, given the orientation of the extinction vector in Figure 27, which is nearly parallel to the age tracks in the population synthesis models, it is clear that the SCs are very likely to be significantly younger and/or more massive than they appear when uncorrected for extinction and reddening due to dust. There are other indicators for high but variable dust extinction: (1) dust lanes evident in the color $(B-I)$ map (Figure 26), (2) the observation that $\sim 90 \%$ of the total luminosity is generated by dust emitting in the thermal infrared (Section 3.5), and (3) evidence for dust entrained in the superwind as far as $\sim 25^{\prime \prime}(15 \mathrm{kpc})$ from the center (Section 3.4.3).

Due to this high variability we can only apply a mean extinction estimate using the following indicators. (1) The emission-line Balmer decrement $(\mathrm{H} \alpha / \mathrm{H} \beta)$ measured from longslit optical spectroscopy resulted in $E(B-V)$ values of $0.72 \mathrm{mag}$ and $0.22 \mathrm{mag}$ for Mrk $266 \mathrm{SW}$ and NE, respectively (Veilleux et al. 1995). ${ }^{32}$ (2) Long-slit spectroscopy along three position angles found $E(B-V)=0.2-0.5$ throughout the inner $\sim 20^{\prime \prime}$ $(10 \mathrm{kpc})$ of Mrk 266 (Wang et al. 1997). These measurements indicate a characteristic extinction of $E(B-V)=0.4\left(A_{V}=\right.$ $1.2 \mathrm{mag}$ ) in Mrk 266, which is similar to the sample mean of

\footnotetext{
32 The identifications of the NE and SW nuclei of Mrk 266 were mistakenly swapped in the tables and figures of Kim et al. (1995) and Veilleux et al. (1995).
} 


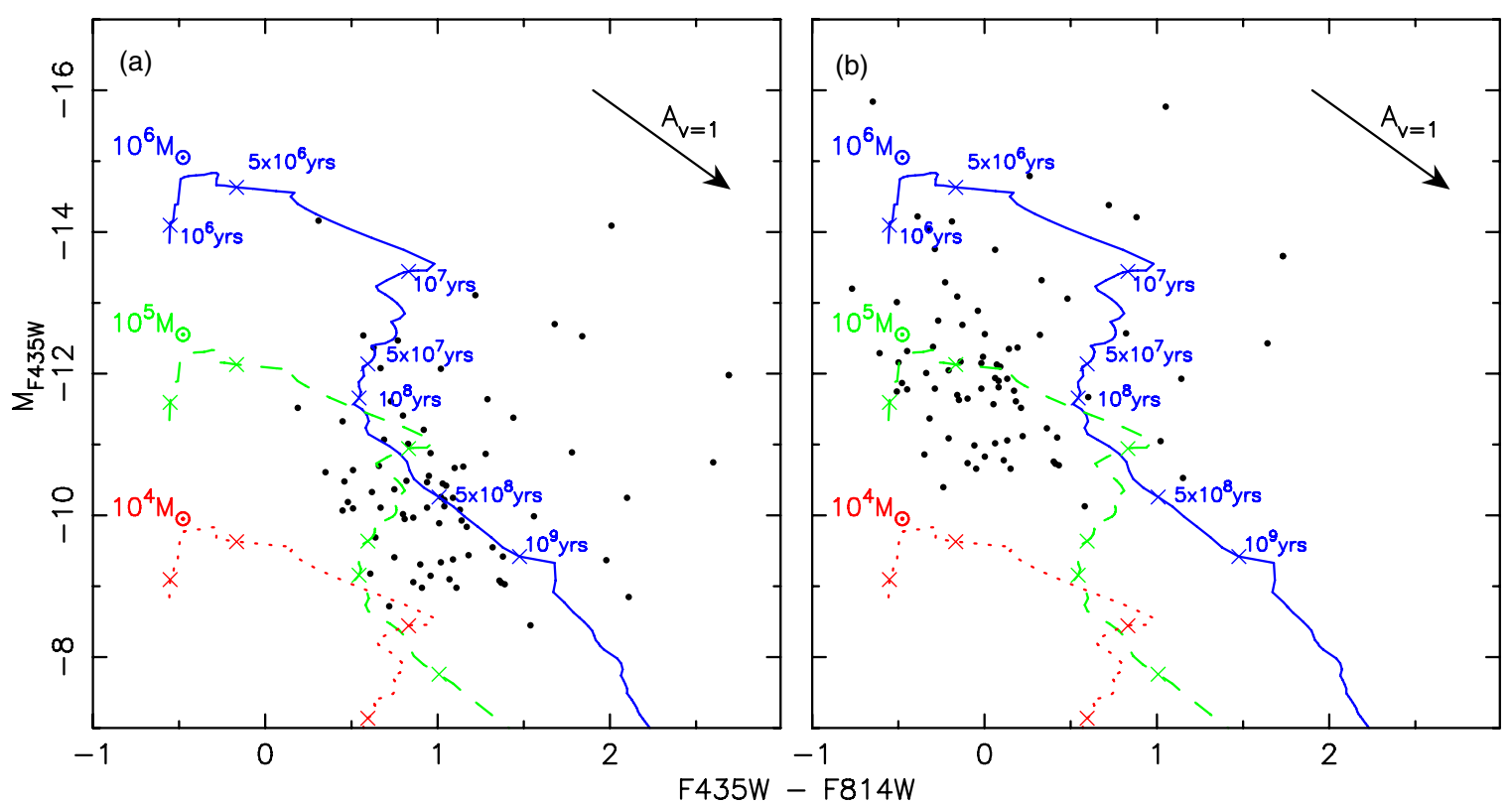

Figure 27. $M_{B}$ vs. $B-I$ color-magnitude diagram for SCs detected in Mrk 266. Evolutionary tracks computed using population synthesis models (Bruzual \& Charlot 2003) assuming an instantaneous starburst and solar metallicity for cluster masses of $10^{4}$ (red), $10^{5}$ (green), and $10^{6}$ (blue) $M_{\odot}$ are shown. Marks on the $10^{6} M_{\odot}$ track indicate the age of the starburst. The data are plotted (a) with no extinction correction, and (b) with a mean extinction correction of $A_{V}=1.2$ mag estimated from various measurements as described in the text. The vector represents 1 mag extinction in the $V$ band. Only SCs with uncertainties in $B-I$ less than 0.2 mag are shown. (A color version of this figure is available in the online journal.)

$E(B-V)=0.5( \pm 0.2)$ mag observed for a large sample of local, luminous starburst galaxies (Calzetti et al. 1994). To estimate the total extinction at particular wavelengths, we assume $R_{V}=3.1$ as found in the general ISM of our Galaxy. However, this can be considered a minimum, because regions containing young SCs in Mrk 266 (and other LIRGs) could have a significantly larger value of $R_{V} \approx 6$ as measured in Galactic star-forming regions such as the Orion Nebula and giant molecular clouds (e.g., McCall 2004).

Application of a mean extinction correction of $A_{V}=1.2 \mathrm{mag}$ to the observed colors (Figure 27(a)) ${ }^{33}$ shifts the points to an intrinsic mean color of $(B-I) \sim 0.0 \mathrm{mag}$ and to a mean luminosity of $M_{B} \sim-12 \mathrm{mag}$ (Figure 27(b)). Comparison of the mean extinction-corrected data to the model curves (Figure 27(b)) leads to the following results for the detected cluster population on average: (1) most of the SCs have ages between 1 and $50 \mathrm{Myr}$; (2) although the current data cannot remove the age/mass degeneracy for the reddest clusters $(B-I \gtrsim 0.5 \mathrm{mag})$, about half of the objects are very blue $(B-I \lesssim 0.2 \mathrm{mag})$, very young (1-5 Myr), and are consistent with masses of $\sim 10^{5} M_{\odot}$; (3) the 11 most luminous SCs have $M_{B} \lesssim-13.5 \mathrm{mag}$ and are located near the (blue) track for $10^{6} \widetilde{M}_{\odot}$ clusters with ages of only $\sim 1-10 \mathrm{Myr}$; (4) the two very luminous objects with $M_{B} \approx-15.8 \mathrm{mag}$ are located $0.75 \mathrm{E}$ (the red cluster with $B-I \approx 1.1$ ) and $0{ }^{\prime} .91 \mathrm{~W}$ (the blue cluster with $B-I \approx-0.6$ ) of the SW nucleus, respectively (see Figure 26 ); and finally (5) if a mean extinction of $A_{V}=1.8 \mathrm{mag}\left(R_{V} \approx 6\right)$ is more applicable than $A_{V}=1.2 \mathrm{mag}$ in regions containing the blue SCs, as argued above, then most of the SCs are on average only $\sim 1$ Myr old and have masses spanning $\sim 10^{5}$ to $10^{6} \mathrm{M}_{\odot}$. These results indicate that the detected SCs are massive and likely relatively young. However, they represent only the "tip of

\footnotetext{
33 The Galactic foreground extinction in the direction of Mrk 266 is only $E(B-V)=0.013$ mag (Schlegel et al. 1998); with caveats documented in NED, this corresponds to $A_{V}=0.04 \mathrm{mag}$.
}

the iceberg" in terms of the extensive starburst which is mostly obscured by dust in optical and UV observations.

\subsubsection{Star Cluster Luminosity Functions}

Figure 28 is a plot of the LF derived for the SCs detected in the $B$ - and $I$-band images. The index $(\alpha)$ of a power-law fit to the LF, after correction of the cluster counts for foreground contamination and completeness using techniques described in Modica et al. (2012) and T. Vavilkin et al. (2012, in preparation), is $\alpha=-2.0 \pm 0.1$ in both the $B$ and $I$ bands.

It is revealing to compare the power-law slope of the SC LF in Mrk 266 with the SC LFs observed in other merger systems. In the well-studied Antennae Galaxy, the cluster LF has $\alpha_{V}=-2.13 \pm 0.07$ (Whitmore et al. 2010). In the earlystage merging LIRG system II Zw 96, $\alpha_{B}=-1.1 \pm 0.3$ was found (Inami et al. 2010). For clusters in two well-known ULIRGs that appear to be in intermediate and late stages of the merger process, the following slopes were measured by Surace et al. (1998): $\alpha_{B}=-1.6 \pm 0.1$ for Mrk 463 and $\alpha_{B}=-1.8 \pm 0.2$ for Mrk 231. For SCs in the late-stage (single nucleus) merger system IC 883, Modica et al. (2012) derived LF indices $\left(\alpha_{B}=-2.2 \pm 0.2\right.$ and $\left.\alpha_{I}=-2.0 \pm 0.2\right)$ that are remarkably similar to the values found in Mrk 266. In another late-stage LIRG merger remnant, NGC 34 (Mrk 938), the SC LF has $\alpha_{V}=-1.75 \pm 0.1$ (Schweizer \& Seitzer 2007). (Both IC 883 and NGC 34 are in the GOALS sample, with $L_{\text {ir }}=5.4 \times 10^{11}$ and $2.5 \times 10^{11} L_{\odot}$, respectively.) It is also noteworthy that the LF slopes in Mrk 266 and these other (U)LIRGs are similar to the LFs of young SCs in the irregular galaxies SMC and LMC, which have mean $\alpha=-2.0 \pm 0.2$ (Gieles et al. 2006). The specific frequency of young SCs is defined as $T_{N}=N_{\mathrm{cl}} \times 10^{\left(0.4\left(M_{B}+15\right)\right)}$, where $N_{\mathrm{cl}}$ is the number of clusters above the specified luminosity limit normalized by the $B$-band luminosity of the host galaxy (e.g., Larsen \& Richtler 1999). Correcting for completeness in the cluster detection, Mrk 266 has $T_{N}=0.91$, which is similar to completeness-corrected 


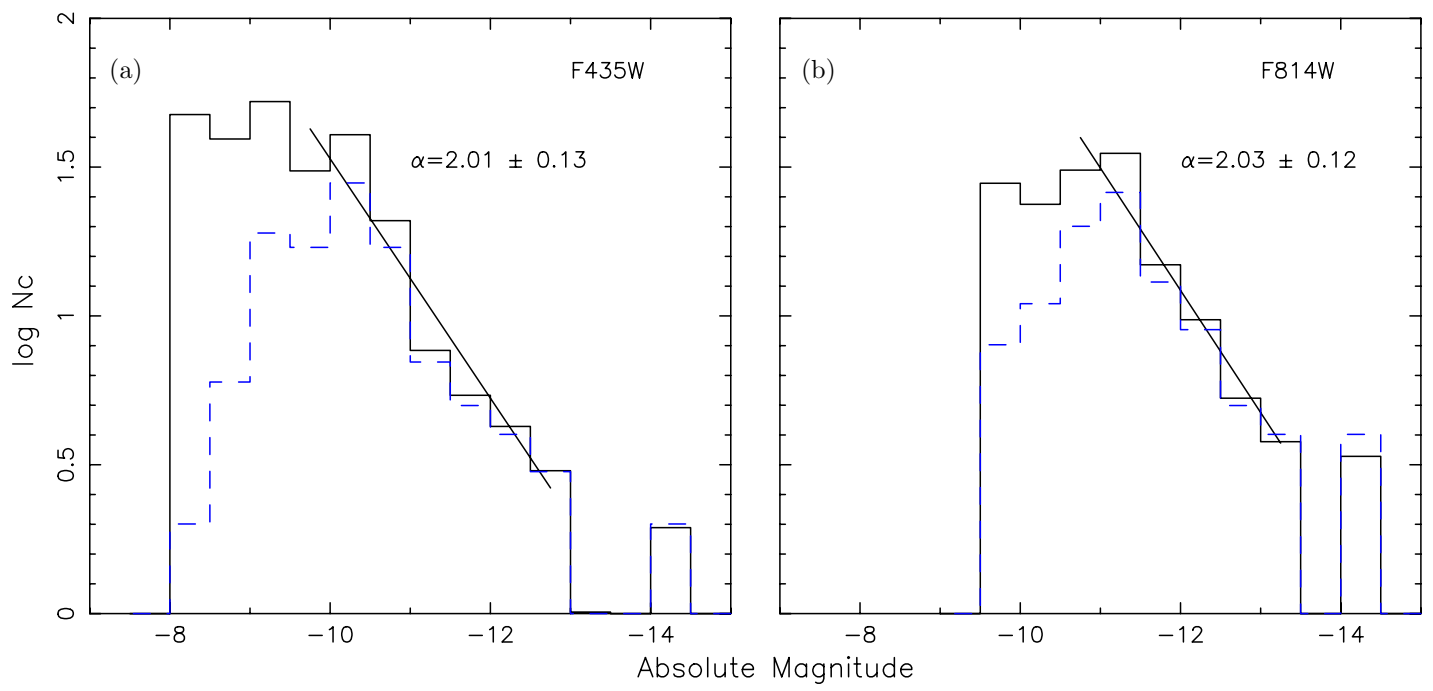

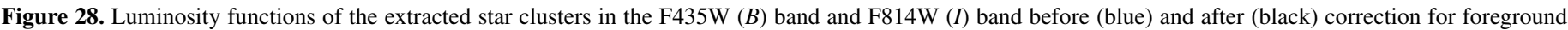

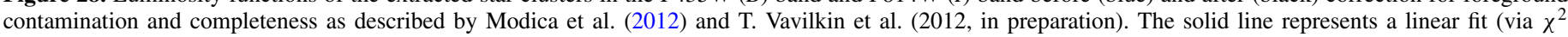

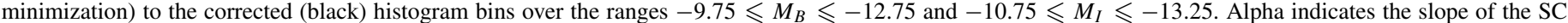
luminosity function in each bandpass over these ranges of $M_{B}$ and $M_{I}$, which have not been corrected for the estimated mean extinction (see the text).

(A color version of this figure is available in the online journal.)

$T_{N}$ values observed in the cluster-rich LIRG sample of T. Vavilkin et al. (2012, in preparation). We conclude that $\alpha$ and $T_{N}$ for SCs in Mrk 266 are similar to other LIRGs.

Various disruption mechanisms have been described in the literature to model the luminosity, mass, and age distributions of SCs in interacting galaxies (e.g., Whitmore et al. 2007). Given that Mrk 266 has one of the most powerful superwinds known in the local universe (Section 3.4), this raises the question of whether SCs are being disrupted by this outflow/feedback process to a larger degree than in systems such as the Antennae with much less powerful superwinds and lower SFRs. The dearth of detected SCs in the interaction zone of Mrk 266 noted above, despite relatively low extinction and molecular gas spanning part of this region (Section 3.7) and despite the presence of $\mathrm{SCs}$ in other regions at equally large distances from the galaxy centers, may be a sign of cluster disruption or suppression of star formation in this narrow band between the two galaxies. A possible explanation would be fast shocks and extensive turbulence at the interface of the colliding galaxies or colliding superwinds expanding from the two galaxies, as discussed in Section 3.3. However, there is no signature of cluster disruption on a global scale in Mrk 266, as might be revealed through an $\alpha$ or $T_{N}$ value that is significantly different than the SC populations observed in (U)LIRGs with less powerful superwinds.

\subsection{Molecular Gas Properties}

\subsubsection{The Integrated Emission from $\mathrm{CO}, \mathrm{NCH}$, and $\mathrm{HCO}^{+}$}

Interferometric observations of Mrk 266 in $\mathrm{HCN}(1-0), \mathrm{HCO}^{+}$ (1-0), and CO (1-0) with the Nobeyama Millimeter Array were presented by Imanishi et al. (2009). In this section, these data are used to explore the distribution of molecular gas with respect to various features in the system and to derive the physical conditions of the cold molecular gas. Figure 29 presents contours of the integrated emission from these three molecular transitions overlaid on images ranging from X-rays through radio continuum. There are intriguing differences between the spatial distributions of these molecular species. Although the SW galaxy contains about five times more flux in CO (1-0) than its companion, the NE galaxy is clearly detected in all three molecular transitions. The bulk of the integrated CO (1-0) emission in the system is aligned with the major axis of the SW galaxy, with a centroid near the X-ray/infrared nucleus. CO (1-0) is also detected in a bridge connecting the two galaxies, which is discussed further below (Section 3.7.2). The HCN gas in the SW galaxy shows double structure with peaks located $\approx 1.2 \mathrm{kpc}\left(2^{\prime \prime}\right)$ to the $\mathrm{NE}$ and $\mathrm{SW}$ of the region where the $\mathrm{CO}(1-0)$ and $\mathrm{HCO}^{+}(1-0)$ are most heavily concentrated at the location of the X-ray/IR/radio nucleus. If this morphology is due to limb brightening in a rotating disk, the $\mathrm{HCN}$ disk is tilted $\sim 10^{\circ}$ with respect to the axis of the $\mathrm{CO}(1-0)$ and $1.6 \mu \mathrm{m}$ emission (Figure 29(e)). Most of the CO emission in the NE galaxy is offset by $\sim 1 \mathrm{kpc}\left(1^{\prime \prime} .5\right)$ to the SE of the X-ray/optical nucleus. The $\mathrm{CO}(1-0)$ in this region is spatially coincident with the peak of the $\mathrm{HCO}^{+}(1-0)$, whereas the dense gas traced by $\mathrm{HCN}$ is concentrated very close to the nucleus about $1 \mathrm{kpc}$ to the NW. Figure 29(c) shows that the centroid of the HCN emission is aligned within 0.2 of the eastern point source in the $H S T$ $B$-band image, which we interpret to be the AGN of Mrk $266 \mathrm{NE}$ (Section 3.2.4).

Applying the formulation of Evans et al. (2005) to compute $L_{\mathrm{CO}}^{\prime}$, and assuming a $\mathrm{CO}$ luminosity to $\mathrm{H}_{2}$ gas mass conversion factor $\alpha=1 M_{\odot}\left(\mathrm{K} \mathrm{km} \mathrm{s}^{-1} \mathrm{pc}^{2}\right)^{-1}$ suggested by observations of ULIRGs (Downes \& Solomon 1998), results in estimates of the warm molecular gas mass for the two galaxies: $3.4 \times 10^{9}$ and $7.0 \times 10^{8} M_{\odot}$ for Mrk $266 \mathrm{SW}$ and NE, respectively. The sum of these components corresponds to a total mass of $M\left(\mathrm{H}_{2}\right)=4.1 \times 10^{9} M_{\odot}$. However, the maps also include an extended component, and the integrated CO (1-0) line flux of the total system is $180 \mathrm{Jy} \mathrm{km} \mathrm{s}{ }^{-1}$ (Imanishi et al. 2009). The corresponding total molecular hydrogen gas mass of Mrk 266 from the Nobeyama observations is thus $M\left(\mathrm{H}_{2}\right)=$ $7.0 \times 10^{9} M_{\odot}$. Within the uncertainties, this is consistent with $206 \mathrm{Jy} \mathrm{km} \mathrm{s}^{-1}$ measured with single-dish observations (Sanders et al. 1986) and a corresponding estimate of $M\left(\mathrm{H}_{2}\right)=2.5 \times$ $10^{9} M_{\odot}$ when adjusted to $\alpha=1$. If the $\mathrm{CO}$ to $\mathrm{H}_{2}$ conversion factor appropriate for Mrk 266 is similar to GMCs in our Milky Way ( $\alpha=4 \pm 1$; Radford et al. 1991), the actual gas masses may be $\sim 4$ times larger than the estimates computed here. (Sanders 


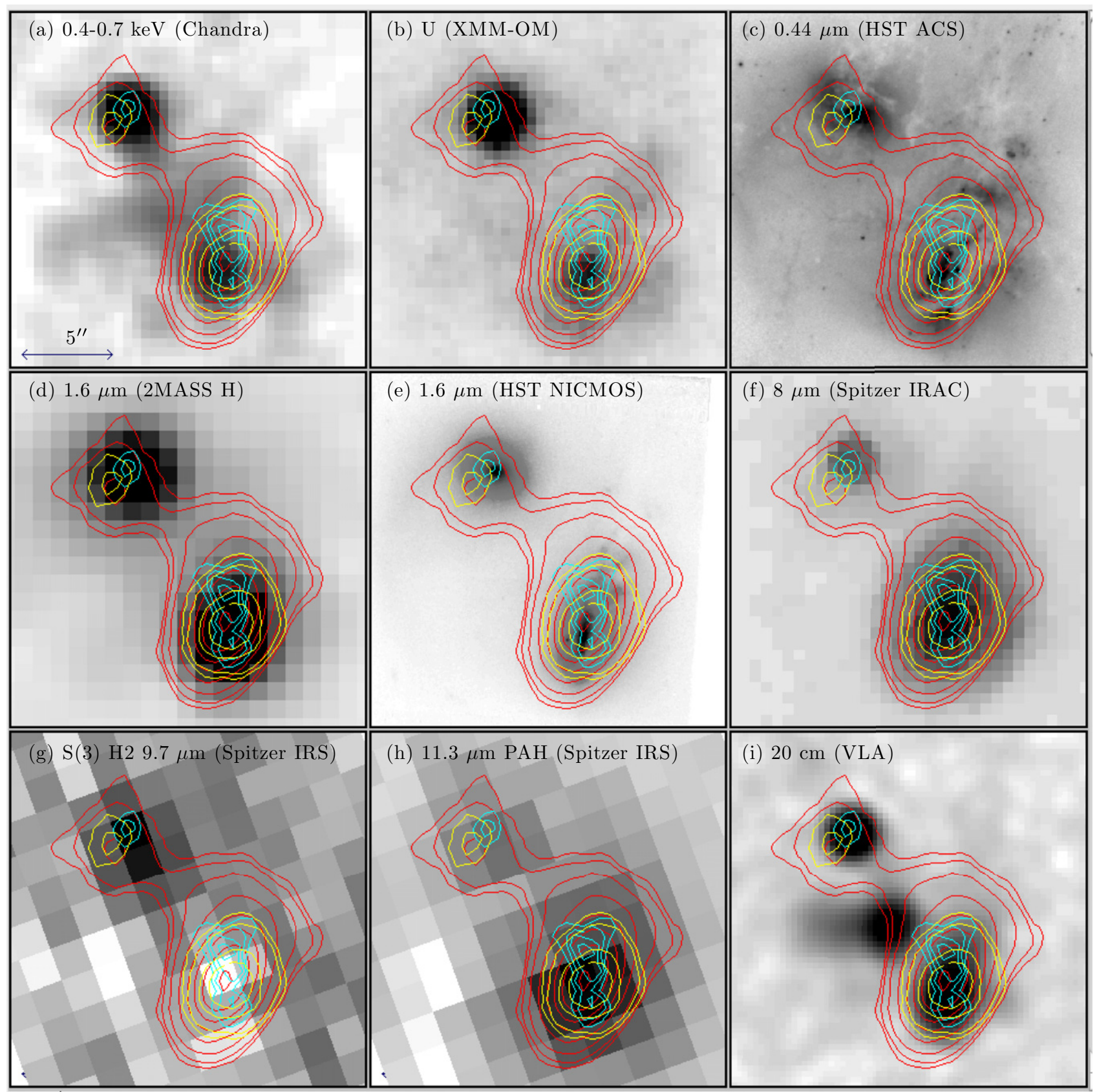

Figure 29. Integrated emission of Mrk 266 in $\mathrm{CO}(1-0)$ (red contours), $\mathrm{HCN}(1-0)$ (cyan contours), and $\mathrm{HCO}^{+}(1-0)$ (yellow contours) with a spatial resolution of 4.'3 × 3".4 (P.A. $=-31.5$ ) from the Nobeyama Millimeter Array (Imanishi et al. 2009) are shown superposed on gray-scale representations of the following images: (a) Chandra 0.4-7 keV X-ray image, (b) XMM-OM $U$ band, (c) HST ACS 0.44 $\mu \mathrm{m}$, (d) $1.6 \mu \mathrm{m}$ (2MASS H), (e) $1.6 \mu \mathrm{m}$ (HST NICMOS), (f) $8.0 \mu \mathrm{m}$ (IRAC), (g) $\mathrm{H}_{2} \mathrm{~S}$ (3) $9.7 \mu \mathrm{m}$ (IRS), (h) $11.3 \mu \mathrm{m}$ PAH (IRS), and (i) $20 \mathrm{~cm}$ (VLA). The 2MASS $1.6 \mu \mathrm{m}$ (H) image is included to show the accuracy of the spatial registration of the $1.6 \mu \mathrm{m}$ NICMOS image. All panels display a field of $20^{\prime \prime} \times 20^{\prime \prime}$. The scale bar is $5^{\prime \prime}$.

(A color version of this figure is available in the online journal.)

et al. 1986 derived $M\left(\mathrm{H}_{2}\right)=1.4 \times 10^{10} M_{\odot}$ assuming $\alpha=4$.) Assuming a weighted mean dust temperature of $50 \mathrm{~K}$ in the $\mathrm{CO}-$ emitting regions based on SED fitting (Section 3.5.1) and using the observed CO (1-0) line widths, applying the formulation of Evans et al. (2005) enables estimation of the radius of the unresolved CO-emitting regions, the dynamical masses of the galaxies, as well as the column densities. These results are presented in Table 9.

\subsubsection{Spatial Distribution and Kinematics of $\mathrm{CO}(1-0)$}

Figure 30 shows the spatial distribution of CO (1-0) in velocity channels ranging from 7850 to $8455 \mathrm{~km} \mathrm{~s}^{-1}$, in judiciously chosen steps varying from 30 to $250 \mathrm{~km} \mathrm{~s}^{-1}$, produced from the FITS channel map provided by Imanishi et al. (2009). The CO (1-0) contours are superposed on the HST NICMOS $1.6 \mu \mathrm{m}$ image for comparison with the highest resolution tracer available for the (old) stellar mass in the galaxies and to minimize confusion from dust obscuration and young star-forming regions that dominate the imagery at shorter wavelengths.

The CO (1-0) emission in Mrk 266 SW shows a strong velocity gradient ranging from $7850 \mathrm{~km} \mathrm{~s}^{-1}$ on the SE side to $8235 \mathrm{~km} \mathrm{~s}^{-1}$ on the NW side of the disk. At progressively higher velocities, the centroid of the $\mathrm{CO}(1-0)$ emission is located at 


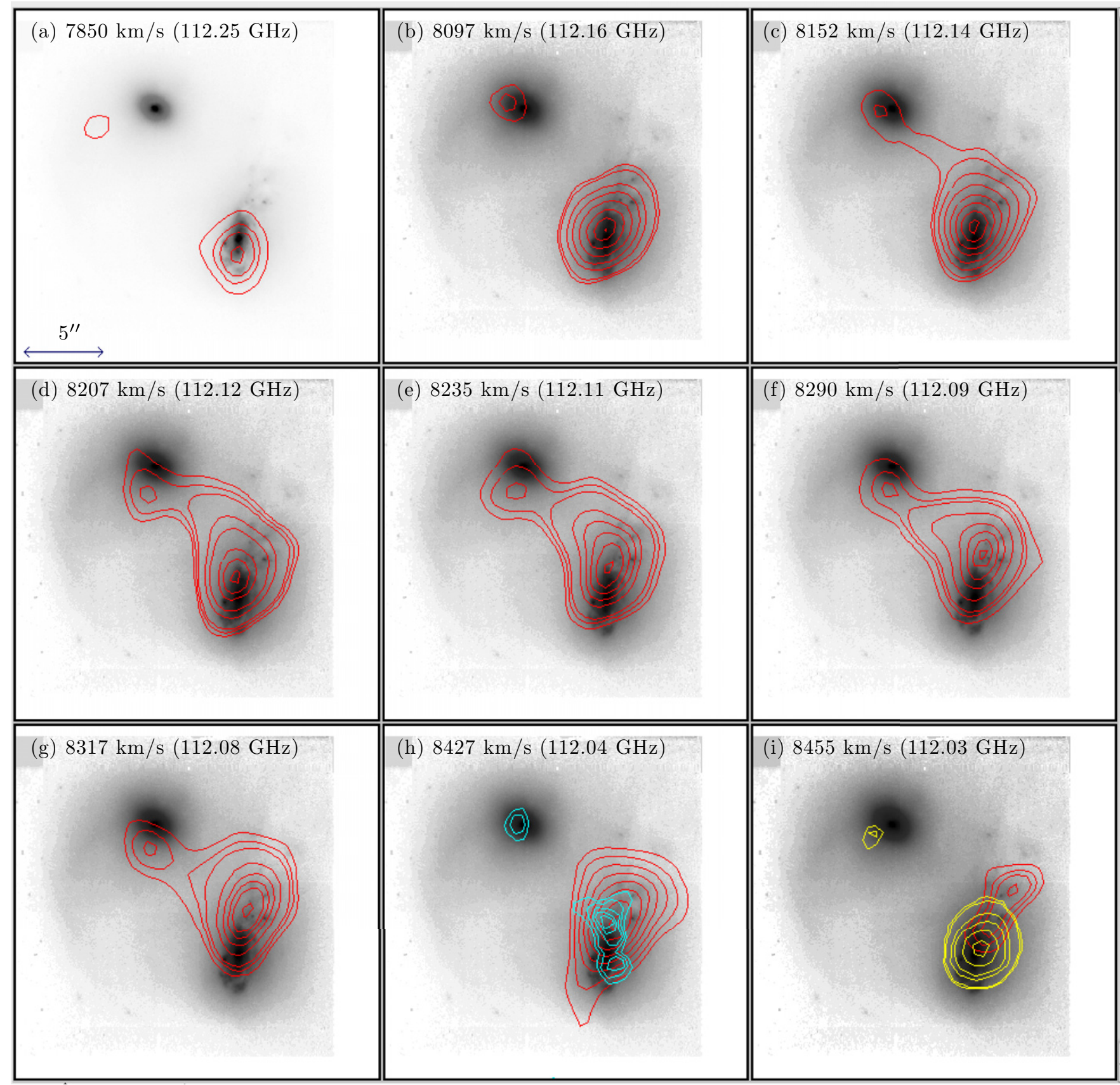

Figure 30. Spatial distribution of the CO (1-0) emission as a function of radial velocity (frequency) using a channel map from the Nobeyama Millimeter Array (Imanishi et al. 2009). The CO data are contoured in red and superposed on a gray-scale representation of the HST $1.6 \mu \mathrm{m}$ image to illustrate the kinematics of the $\mathrm{CO}$ with respect to the old stars in the galaxies. For comparison, the integrated emission from $\mathrm{HCN}(1-0)$ is plotted (cyan contours) in panel (h), and the integrated emission from $\mathrm{HCO}^{+}(1-0)$ is shown (yellow contours) in panel (i). The mean radial velocity (and frequency) is shown in each panel. All panels display a $24^{\prime \prime} \times 24^{\prime \prime}$ field, and the scale bar is $5^{\prime \prime}$.

(A color version of this figure is available in the online journal.)

larger radii, extending as far as $\approx 5^{\prime \prime}(3 \mathrm{kpc}) \mathrm{NW}$ from the SW nucleus. The molecular gas in this off-nuclear region has a radial velocity $\approx 450 \mathrm{~km} \mathrm{~s}^{-1}$ greater than the SW nucleus, and this gas coincides spatially with the most luminous region of off-nuclear star formation that is prominent in optical and UV images (see Figure 8). Although the density of SCs is not significantly higher than in the surrounding areas, this region contains many of the most luminous SCs (Figure 25).

The low $L_{\mathrm{CO}} / L_{\text {ir }}$ ratio in Mrk $266 \mathrm{NE}$ is consistent with its apparent near-IR Hubble type of S0/a (pec), suggesting that its pre-merger gas content was relatively low and incapable of sustaining a high SFR. Figure 30 shows that a large quantity of molecular gas detected over the velocity range $8150-8320 \mathrm{~km} \mathrm{~s}^{-1}$ is bridging between the galaxies. The highest concentration of $\mathrm{CO}$ on the $\mathrm{NE}$ side of the bridge is centered $\approx 1 \mathrm{kpc}\left(1^{\prime \prime} .5\right)$ to the SE of the nucleus of Mrk 266 NE. As shown in Figure 29(i), the $\mathrm{CO}(1-0)$ in this off-nuclear region coincides with the peak of the $\mathrm{HCO}^{+}(1-0)$ emission, whereas the $\mathrm{HCN}$ (1-0) emission is concentrated at the NE nucleus. This result is consistent with research that has called into question the use of $\mathrm{HCN}$ as a tracer of dense gas fueling star formation, suggesting that in some cases HCN is energized by an X-ray Dissociation 
Table 9

Molecular Gas Properties of Mrk 266

\begin{tabular}{lccc}
\hline \hline $\begin{array}{l}\text { Parameter } \\
(1)\end{array}$ & $\mathrm{SW}$ & $\mathrm{NE}$ & $\begin{array}{c}\text { Total } \\
(4)\end{array}$ \\
\hline $\mathrm{CO}(1-0)$ flux $\left(\mathrm{Jy} \mathrm{km} \mathrm{s}^{-1}\right)^{\mathrm{a}}$ & 88 & $(3)$ & 180 \\
$\mathrm{CO}(1-0)$ FWHM $\left(\mathrm{km} \mathrm{s}^{-1}\right)^{\mathrm{a}}$ & 500 & 250 & $\ldots$ \\
$L_{\mathrm{CO}}^{\prime}\left(\mathrm{K} \mathrm{km} \mathrm{s} \mathrm{kc}^{-1} \mathrm{pc}^{2}\right.$ & $3.4 \times 10^{9}$ & $7.0 \times 10^{8}$ & $7.0 \times 10^{9}$ \\
$M\left(\mathrm{H}_{2}\right)\left(M_{\odot}\right)$ & $3.4 \times 10^{9}$ & $7.0 \times 10^{8}$ & $7.0 \times 10^{9}$ \\
$\mathrm{R}(\mathrm{CO})(\mathrm{pc})$ & 208 & 134 & $\ldots$ \\
$M_{\text {Dynamical }}\left(M_{\odot}\right)$ & $1.2 \times 10^{10}$ & $1.9 \times 10^{9}$ & $\ldots$ \\
$M\left(\mathrm{H}_{2}\right) / M_{\text {Dynamical }}$ & 0.28 & 0.37 & $\ldots$ \\
Column density $\left(\mathrm{cm}^{-2}\right)$ & $1.6 \times 10^{24}$ & $7.9 \times 10^{23}$ & $\ldots$ \\
\hline
\end{tabular}

Notes. Column 1: observed or derived parameter. Columns 2-4: for the SW galaxy, NE galaxy, and the total system, respectively, the value of the parameter in Column 1.

a The CO (1-0) line fluxes and line widths are from Imanishi et al. (2009); all other parameters are derived here for the first time.

Region associated with an AGN (e.g., Graciá-Carpio et al. 2008).

The distribution of CO (1-0) in Mrk 266 suggests the system is currently in a short-lived phase in which $\sim 40 \%$ of the cold molecular gas is falling toward the center of mass of the system or transferring between the galaxies along a tidal bridge as predicted by merger simulations (e.g., Cox et al. 2006). Mrk 266 appears to be in an evolutionary stage similar to three other LIRGs in the GOALS sample that have a large fraction of their total CO emission located between the nuclei: VV 114 (Yun et al. 1994), NGC 6090 (Bryant \& Scoville 1999), and NGC 6240 (Engel et al. 2010). However, unlike VV 114 where the $1.4 \mathrm{GHz}$ radio continuum emission has a spatial distribution similar to the $\mathrm{CO}$, the enhanced radio continuum emission between the nuclei of Mrk 266 peaks $\approx 2^{\prime \prime}(1.2 \mathrm{kpc})$ south of the CO bridge (Figure 29(i)). Another difference is that whereas the bulk of the $\mathrm{HCO}^{+}$in VV 114 is located between the nuclei (Imanishi et al. 2007), in Mrk 266 the $\mathrm{HCO}^{+}$is still bound to the galaxies.

\subsection{Wider Implications}

The preceeding sections have shown that Mrk 266 exhibits the simultaneous occurrence of a variety of phenomena including dual AGNs, an outflow containing a likely radiative bow shock in the narrow-line region of the NE nucleus, a starburst with a population of young SCs, a galactic-scale "blow-out" expelling dust in a superwind, shocked gas between the galaxies, and molecular gas falling toward the center of mass or transferring between the galaxies. In this closing section, Mrk 266 is compared with other local major mergers and (U)LIRGs, and we attempt to place the system into a tentative sequence with a small number of other confirmed dual AGNs (with kiloparsec scale nuclear separations) that may represent stages in the formation of binary AGNs (accreting SMBHs with parsec-scale orbital separations).

\subsubsection{Emission between Interacting Galaxies}

Interacting systems exhibiting substantial radio continuum, $\mathrm{X}$-ray, or molecular gas emission between the galaxies are rare, but a few have been studied in detail. By comparing these systems with Mrk 266 we may be able to gain further insight into this phenomenon.

The Taffy Galaxy ( $V V 254$ ). This interacting pair has $\approx 50 \%$ of its total $1.5 \mathrm{GHz}$ flux emerging between the companions, which has been interpreted as synchrotron radiation from cosmic-ray electrons trapped in magnetic field lines and stretched like taffy as the galaxies pass through each other (Condon et al. 1993). Imaging in 450 and $850 \mu \mathrm{m}$ continuum emission and in multiple transitions of $\mathrm{CO}$ indicate the bridge between the galaxies consists of interstellar dust and gas extruded from the galaxy disks via direct cloud-cloud collisions (Zhu et al. 2007). Midinfrared observations have detected substantial PAH emission in the bridge (Jarrett et al. 1999).

The regions between the components of Taffy and Mrk 266 both contain large fractions of their total radio continuum and $\mathrm{CO}$ emission, yet they contain little or no evidence for luminous star formation. However, unlike Taffy, there is no detected infrared enhancement between the galaxies of Mrk 266. In addition, while the radio continuum and $\mathrm{CO}$ emission are roughly spatially aligned and uniformly distributed between the Taffy galaxies, in Mrk 266 the central radio emission is much more centrally concentrated and the bridge of $\mathrm{CO}(1-0)$ emission is spatially decoupled from the radio continuum and $\mathrm{X}$-ray emission. The absence of a discrete mid-IR or far-IR source between the galaxies of Mrk 266 suggests that shocks may have destroyed most of the dust grains in this region. The collision in the Taffy system was estimated to occur $\sim 2 \times 10^{7} \mathrm{yr}$ prior to its current configuration (Condon et al. 1993). Its global SFR is not significantly higher than in non-interacting spiral galaxies (Zhu et al. 2007), and no evidence for an AGN has been published for either galaxy.

The Antennae (NGC 4038/4039). This galaxy pair is well known for having $\sim 30 \%$ of its total bolometric luminosity, $\sim 40 \%$ of its total CO (1-0) flux (Zhu et al. 2003), and a high concentration of young SCs (Whitmore et al. 1999) located in an off-nuclear region between the colliding disks. We have shown that in Mrk 266 the molecular gas bridging the galaxies is decoupled from the central radio/X-ray source, and there is no evidence for star formation between the galaxies. Compared to Mrk 266, the Antennae exhibit: (1) 5 times lower luminosity in the far-infrared and in CO (1-0), (2) a cooler effective dust temperature, (3) a large region of diffuse X-rays, but a weaker superwind, (4) a pair of long tidal tails rather than diffuse, asymmetric tidal debris, and (5) low-luminosity LINER and starburst (H II) nuclei.

Early simulations suggested we are viewing the Antennae galaxies soon after their first encounter (e.g., Dubinski et al. 1996), but a recent model that accounts for the starburst in the overlap region constrains the system to $\sim 40$ Myr after the second encounter and only $\sim 50 \mathrm{Myr}$ before the galaxy centers fully merge (Karl et al. 2010). The close projected separation and small relative velocity of the nuclei imply Mrk 266 is in a similar stage of the merger process as the Antennae. The lack of well-defined tidal tails in Mrk 266 may be due to any number of conditions, including a relatively small disk mass in the NE galaxy (Section 3.1.1), a retrograde encounter or an orbital geometry (e.g., a smaller perigalactic distance) that produces more diffuse tidal debris (Dubinski et al. 1996), or a large ratio of dark matter halo mass to visible (bulge plus disk) mass that can cause tails produced early in the encounter to fall back onto the galaxies quickly, resulting in late-stage tidal debris resembling loops and shells (Mihos et al. 1998). It is noteworthy that the extent of the diffuse optical emission in Mrk $266(\approx 103 \mathrm{kpc})$ is similar to the end-to-end length of the tails in the Antennae. A detailed simulation of kinematic data for Mrk 266 is needed to constrain its true dynamical age.

NGC 6240. Mrk 266 appears to be in a similar evolutionary stage as the well-known system NGC 6240, in which X-ray 
observations by Chandra have also revealed dual AGNs (Komossa et al. 2003) and a large fraction of the molecular gas is located between the nuclei (Tacconi et al. 1999). Although the total infrared luminosity is about half that of NGC 6240, Mrk 266 has a much warmer dust temperature $\left(f_{60 \mu \mathrm{m}} / f_{100 \mu \mathrm{m}}=\right.$ $0.72)$ compared with NGC $6240\left(f_{60 \mu \mathrm{m}} / f_{100 \mu \mathrm{m}}=0.26\right)$. Likewise, decomposition of the infrared SEDs indicates warm (cool) dust components of $225 \mathrm{~K}(73 \mathrm{~K})$ in Mrk 266 compared to $193 \mathrm{~K}(61 \mathrm{~K})$ in NGC 6240 (Section 3.5.1). The $0.4-10 \mathrm{keV}$ $\mathrm{X}$-ray luminosity of Mrk 266, $9.5 \times 10^{41} \mathrm{erg} \mathrm{s}^{-1}$, is $57 \%$ that of NGC 6240, and within the uncertainties of the X-ray flux absorption corrections their intrinsic $L_{x} / L_{\text {ir }}$ ratios are similar $\left(5-7 \times 10^{-4}\right)$. The projected separation of the nuclei in NGC 6240 is $\approx 1 \mathrm{kpc}\left(1^{\prime \prime} .8\right)$.

Stephan's Quintet. This is a well-studied compact group containing a large intergalactic shock region with substantial radio continuum, X-rays, infrared, and molecular gas emission. Unlike Mrk 266, the main shock region of Stephan's Quintet exhibits broad $\left(870 \mathrm{~km} \mathrm{~s}^{-1}\right)$ rotational lines of warm $\mathrm{H}_{2}$ with an order of magnitude higher surface brightness than the soft X-ray emission (Appleton et al. 2006). This dichotomy may be explained by a difference in the shock conditions in the two systems. Detailed modeling suggests the shock velocity in Stephan's Quintet is $\leqslant 300 \mathrm{~km} \mathrm{~s}^{-1}$ (Cluver et al. 2010). As noted by Davies et al. (2000), if the intergalactic shock front in Mrk 266 is produced by the collision of superwinds expanding outward from the two galaxies at $\sim 300 \mathrm{~km} \mathrm{~s}^{-1}$, adding this $\Delta V \sim 600 \mathrm{~km} \mathrm{~s}^{-1}$ to the collisional velocity of the two galaxies can produce a net shock velocity approaching $\sim 1000 \mathrm{~km} \mathrm{~s}^{-1}$. This would heat the gas far above the dissociation temperature of $\mathrm{H}_{2}$ (Lim 2003), which is consistent with a temperature of $\sim 10^{7} \mathrm{~K}$ deduced from modeling of the soft X-ray data (Section 3.3.2). Another difference between these systems is that although $\sim 40 \%$ of the CO (1-0) in Mrk 266 is located in a bridge between the galaxies where little or no star formation is taking place, in Stephan's Quintet CO (1-0) is detected in regions where star formation is occurring as traced by $24 \mu \mathrm{m}$ dust emission and UV continuum emission from young, massive stars (Cluver et al. 2010).

In summary, the radio continuum and X-ray emission between the galaxies in Mrk 266 is more similar to the large-scale shock detected in Stephan's Quintet than the off-nuclear star formation observed in the Antennae. The lack of bright $\mathrm{H}_{2}$ line emission and infrared emission from dust grains in the central region of Mrk 266 may be due to the presence of a very fast shock that effectively destroys most of the dust grains, sublimates the molecular gas, and inhibits star formation. The ridge of $\sim 10^{7} \mathrm{~K}$ gas between the galaxies of Mrk 266, which is nearly $\sim 10$ times hotter than in other regions of the diffuse $\mathrm{X}$-ray nebula and has a correspondingly short cooling time, is consistent with this scenario. Since the region with enhanced soft X-rays and radio continuum emission between the galaxies is not well aligned with the molecular gas bridge (Figure 29) and the physical processes generating these features are likely quite different (shocks generated via superwinds and gas transfer via gravitation tidal forces), we suspect that these two shortlived phases are not directly linked. However, their simultaneous appearance is not a mere coincidence, as both phenomena are causally related to the ongoing galaxy merger.

\subsubsection{Will Mrk 266 Become a ULIRG?}

It is well established that most local LIRGs and effectively all ULIRGs involve interacting and merging gas-rich spiral galaxies (e.g., Sanders \& Mirabel 1996), and the ULIRG phenomenon occurs predominantly in late-stage mergers when dust is heated to high temperatures by being transported by gravitational torques (with the rest of the ISM) close to the central power sources (e.g., Mazzarella et al. 1991a; Mihos \& Bothun 1998). Clearly ULIRGs must pass through a LIRG phase as the SFR and/or AGN fueling rate increases the dust heating to produce a luminosity of $L_{\mathrm{ir}} \gtrsim 10^{12} L_{\odot}$. At least two lines of evidence — one based upon merger simulations and one based on molecular gas properties-suggest that Mrk 266 will transform into a ULIRG.

Numerical simulations show that the timescale for major mergers (from initial approach through final coalescence of the nuclei) averages $\sim 2$ Gyr, but spans 1-8 Gyr, depending on the circularity of the orbit, the virial radius, and circular velocity (e.g., Jiang et al. 2008). Comparison of the observed properties of Mrk 266 with hydrodynamical simulations (Barnes \& Hernquist 1996; Mihos \& Hernquist 1996; Hopkins et al. 2006; Cox et al. 2006) suggests we are viewing this system about $50 \%$ to $70 \%$ of the way through the merger process. The presence of an asymmetric halo of low surface brightness optical emission spanning over $100 \mathrm{kpc}$, a relatively small projected nuclear separation of $6 \mathrm{kpc}$, and a low relative velocity of the nuclei of $\Delta V=135 \mathrm{~km} \mathrm{~s}^{-1}$ (Khachikian et al. 2006) indicate the two galaxies are likely undergoing their second or third encounter, with each passage damping the relative velocities of the nuclei, strong dynamical friction causing the orbit to decay, and tidal torques driving strong inflows of gas. As pointed out by Mihos (1999), the timescale for violent relaxation is $\gtrsim 1$ Gyr in the outer regions (low $V_{c}$ ), but only $\sim 100$ Myr in the inner regions (high $V_{c}$ ). Thus, depending on whether the nuclei are on the verge of free fall (which can occur as rapidly as $\approx 1 / 4$ of a rotation period) or whether they will orbit one more time before fully merging, the estimated time to coalescence is about 50-250 Myr. (We are referring here to the merger of the stellar systems, not the SMBHs. The latter is discussed in Section 3.8.3.) Based on Monte Carlo simulations of merger models to match the observed distribution of nuclear separations in ULIRGs (Murphy et al. 1996), Mihos (1999) concluded that most ULIRGs are in the final $20 \%$ of the merger process. Thus, Mrk 266 appears to be in an interesting evolutionary stage when the nuclei are about to enter the final phase of coalescence characteristic of ULIRGs.

The total cold molecular gas mass of Mrk 266 is similar to that of ULIRGs observed locally and at high redshifts (e.g., Solomon \& Vanden Bout 2005). Given that $\approx 40 \%$ of the total $\mathrm{CO}(1-0)$ emission is in a bridge between the galaxies, there is a large reservoir of cold molecular gas available to form more stars or to fuel the AGNs as the galaxies and nuclei fully coalesce. With $L_{\mathrm{CO}}^{\prime}=6.7 \times 10^{9} L_{\odot}$ and $L_{\mathrm{ir}} / L_{\mathrm{CO}}^{\prime}=50.4$, Mrk 266 falls directly on the well-known correlation between $\log \left(L_{\mathrm{ir}} / L_{\mathrm{CO}}^{\prime}\right)$ and $\log \left(L_{\mathrm{ir}}\right)$ (Solomon \& Vanden Bout 2005). Within the evolutionary scenario of Sanders et al. (1988a, 1988b), we expect Mrk 266 will evolve into a ULIRG initially with a relatively "cool" global far-infrared dust temperature similar to Arp 220, followed by a "warm" ULIRG phase exemplified by Mrk 463 (Mazzarella et al. 1991b; Bianchi et al. 2008). Given the new evidence for dust entrainment in the extensive superwind of Mrk 266 (Section 3.4.3), this system illustrates that the "blow-out phase" when large quantities of dust are expelled to reveal previously obscured optical/UV AGNs can begin during an intermediate stage of the merger before the nuclear coalescence phase when ULIRGs are most likely to occur. 
ULIRGs have a much lower space density than LIRGs locally and at intermediate redshifts (Le Floc' $h$ et al. 2005). In addition, the mean luminosity of ULIRGs in the local universe is lower than ULIRGs and submillimeter galaxies at high redshifts (e.g., Solomon \& Vanden Bout 2005). The resolution of these observations may well lie in the pre-merger gas content of the participant galaxies. The higher that gas content, the larger the burst of star formation and subsequent dust heating. In the case of Mrk 266, the NE component contains $\approx 5$ times less molecular gas than its companion; this is consistent with its apparent morphological type (S0/a) and correspondingly high bulge-to-disk ratio found at $1.6 \mu \mathrm{m}$. Using the well-established correlation of $L_{\mathrm{CO}}$ with $L_{\text {ir }}$ (Young et al. 1986), a merging pair involving two late-type spirals with molecular gas masses similar to Mrk $266 \mathrm{SW}$ (SBb) would have $L_{\mathrm{CO}}$ a few times higher than is observed in the actual Mrk $266(\mathrm{SBb}+\mathrm{S} 0 / \mathrm{a})$ pair, resulting in a total $L_{\mathrm{ir}}$ at the high end of the local ULIRG distribution (Solomon \& Vanden Bout 2005).

In the hierarchical model, a galaxy's Hubble type is a byproduct of its merger history (e.g., Schweizer 2000). In this scenario, although merging played an important role in galaxy evolution over all cosmic time, high- $z$ galaxies and protogalaxies were very different than those observed at intermediate and low redshifts, and prior to $z \sim 1$ the Hubble sequence was not yet in place. However, from $z \sim 1$ to the present, because many late-type spiral galaxies merged and transformed into early-type spirals, S0, or elliptical galaxies, the fraction of late-type spirals has decreased substantially (e.g., Oesch et al. 2010). Locally at $z \sim 0$, this makes pairings of gas-rich, latetype galaxies less likely than pairings of one late-type with one early-type galaxy (or of two early-type galaxies). Therefore, at low redshifts a smaller fraction of major mergers will have sufficient gas to fuel star formation or AGN accretion at the level required to reach the ULIRG threshold during early to intermediate phases of the merger. As argued above, however, this would not prevent a ULIRG from developing later if one of the companions has $\gtrsim 7 \times 10^{9} M_{\odot}$ of molecular gas available to be driven toward the center of mass of the system to fuel two massive AGNs as they inspiral toward final coalescence. Mrk 266 is apparently in this physical situation.

\subsubsection{Evolution from Dual to Binary AGNs}

In this final section, we turn attention to the significance of Mrk 266 in context with other confirmed dual AGN systems in an attempt to understand the low detection rate of binary AGNs with sub-parsec scale orbits found in recent surveys. We also propose a putative evolutionary sequence involving a quantitative parameter available for a small number of major merger systems with confirmed dual AGNs and candidate binary AGNs.

Current theory predicts the following evolutionary sequence (e.g., Colpi \& Dotti 2009, and references therein): (1) pairs of massive galaxies separated by many times the diameter of their luminous disks and bulges will merge in $\sim 1$ Gyr (a few dynamical timescales) due to dynamical friction between their massive dark matter halos, (2) the two pre-existing SMBHs in their cores (with $M_{\mathrm{SMBH}} / M_{\text {bulge }} \approx 0.1 \%$ ) will form $d u a l$ $A G N s$ as tidal torques drive the ISM inward to fuel accretion at a higher rate than existed prior to the encounter; during this stage the nuclei are merging from a separation of $\gtrsim 1 \mathrm{kpc}$ to $\sim 10 \mathrm{pc}$, (3) in many cases conditions may be conducive to forcing the black holes pairs to inspiral over a period of $\sim 100 \mathrm{Myr}$ to a separation of $\lesssim 10 \mathrm{pc}$, at which point they enter
Keplerian orbits and may be detectable as binary $A G N s$, (4) the SMBHs will eventually either completely coalesce or else recoil, resulting in the most powerful sources of gravitational waves predicted to exist. Considering the relatively small number of dual AGNs confirmed to date, it is tempting to conclude that it is rare for both SMBHs to have accretion rates high enough to power two luminous AGNs simultaneously. However, given that the column density of gas and dust in (U)LIRGs is high enough to make the central $\sim 100 \mathrm{pc}$ optically thick in the mid-infrared, and even in the far-infrared in many cases (e.g., Thompson et al. 2005), many more dual AGNs will likely be detected with observations in other spectral regions. Indeed, X-ray surveys suggest $\approx 60 \%$ of optically classified LINERs are true AGNs (Gonzalez-Martin et al. 2006), and high-resolution radio continuum imaging finds a high fraction (74\%) of AGNs among optically classified LINERs in the 1 Jy ULIRG sample (Nagar et al. 2003).

Along with Mrk 266 and NGC 6240, the following local (U)LIRGs have also been confirmed to contain dual AGNs based on detection of two compact, luminous sources of hard X-rays: Mrk 171 (Arp $299=$ NGC 3690 + IC 694) (Ballo et al. 2004) and Mrk 463 (Bianchi et al. 2008). Mrk 171 is similar to NGC 6240 in terms of infrared luminosity $\left(L_{\mathrm{ir}}=8.5 \times 10^{11} L_{\odot}\right)$ and projected nuclear separation $\left(0.9 \mathrm{kpc}, 3^{\prime \prime} .8\right)$. However, with $L_{h x}(2-10 \mathrm{keV})=2.7 \times 10^{41} \mathrm{erg} \mathrm{s}^{-1}$ (uncorrected for absorption), Mrk 171 has $L_{h x} / L_{\mathrm{ir}}=8.4 \times 10^{-5}$, which is an order of magnitude smaller than Mrk 266 and NGC 6240. With $L_{h x}(2-10 \mathrm{keV})=3.8 \times 10^{42} \mathrm{erg} \mathrm{s}^{-1}$, Mrk 463 has a hard X-ray luminosity 9 times greater than Mrk 266 and 16 times greater than Mrk 171. Mrk 463 has $L_{h x} / L_{\mathrm{ir}}=1.9 \times 10^{-3}$, which is 2-5 times larger than NGC 6240 and Mrk 266, and 23 times larger than Mrk 171. A remarkable dual AGN is 3C 75, in which both nuclei are emitting powerful double-sided radio jets (Owen et al. 1985); this system consists of a pair of elliptical galaxies with projected separation $6.4 \mathrm{kpc}\left(14^{\prime \prime}\right)$.

There is growing evidence that the current small number of confirmed dual AGNs will increase, primarily with observations of hard X-rays. For example, in a local sample of hard X-rayselected AGNs, $10 \%$ have been found to reside in dual systems with separations $<100 \mathrm{kpc}$ (Koss et al. 2012). ${ }^{34}$ Dual AGNs and binary AGN candidates are also being found in surveys probing to higher redshifts. The $z=0.36$ system COSMOS J100043.15+020637.2 has been confirmed to contain two luminous AGNs with a separation of $2.5 \mathrm{kpc}\left(0^{\prime \prime} .5\right)$ (Comerford et al. 2009). In CXOC J100043.1+020637, Civano et al. (2010) have interpreted the presence of optical morphology indicating a major merger, a large velocity offset, and an X-ray iron line with an inverted P-Cygni profile as a recoiling SMBH or a slingshot effect in a triple system. The published SED indicates $L_{h x} / L_{\mathrm{ir}} \approx 0.018$, which is orders of magnitude larger than in the local (U)LIRGs. Four more dual AGNs with kiloparsec-scale separations and $0.13<z<0.21$ have also been discovered recently in the SDSS (Liu et al. 2010).

What appears to be the first bona fide binary AGN was discovered in $4 \mathrm{C}+37.11$, which contains two compact radio sources with a projected separation of only 7.5 mas ( $7 \mathrm{pc}$ ). Based on the radio source compactness, variability, motion in the jets, and spectral shapes, Rodriguez et al. (2006) concluded that both

\footnotetext{
34 However, it should be noted that many pairs with such wide separations may not be gravitationally bound, and a hard X-ray-selected sample is naturally biased toward a much higher AGN fraction than an infrared-selected sample. The number of confirmed dual AGNs is currently less than $3 \%$ of the GOALS sample.
} 
Table 10

Dual to Binary AGN Evolution Based on $L_{h x} / L_{\mathrm{ir}}$

\begin{tabular}{|c|c|c|c|c|c|c|c|c|}
\hline Object & (2) & $\begin{array}{c}D_{L} \\
(\mathrm{Mpc}) \\
(3)\end{array}$ & $\begin{array}{l}\text { Sep } \\
\left({ }^{\prime \prime}\right) \\
(4)\end{array}$ & $\begin{array}{c}\text { Sep } \\
(\mathrm{kpc}) \\
(5)\end{array}$ & $\begin{array}{c}\log \left(\frac{L_{\text {ir }}}{L_{\odot}}\right) \\
(6)\end{array}$ & $\begin{array}{c}\log \left(\frac{L_{h x}}{L_{\mathrm{ir}}}\right) \\
(7)\end{array}$ & Notes & References \\
\hline Mrk 171 (NGC 3690 + IC 694) & 0.01041 & 51.1 & 3.8 & 0.9 & 11.88 & -4.1 & $\ldots$ & 1 \\
\hline Mrk 266 (NGC 5256) & 0.02786 & 129 & 10 & 6.0 & 11.53 & -3.5 & $\ldots$ & 2 \\
\hline NGC 6240 & 0.02448 & 116 & 1.8 & 0.9 & 11.93 & -3.1 & $\ldots$ & 3 \\
\hline Mrk 463 & 0.05036 & 233 & 3.8 & 3.8 & 11.77 & -2.8 & Young radio jet? & 4 \\
\hline $3 \mathrm{C} 75$ & 0.02315 & 97.9 & 14 & 6.4 & $\ldots$ & $\ldots$ & Twin radio jets & 5 \\
\hline COSMOS J100043.15+020637.2 & 0.36060 & 1944 & 0.5 & 2.5 & 11.59 & -1.7 & Recoiling SMBH? & 6 \\
\hline $4 C+37.11$ & 0.05500 & 242 & 0.0073 & 0.008 & $\ldots$ & $\ldots$ & Binary AGN & 7 \\
\hline SDSS J153636.22+044127.0 & 0.38930 & 2133 & $1.0 ?$ & $10^{-4}$ or 5.1 & $\ldots$ & $\ldots$ & Binary or dual AGN? & 8,9 \\
\hline
\end{tabular}

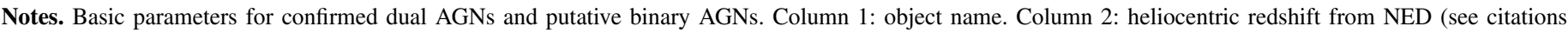

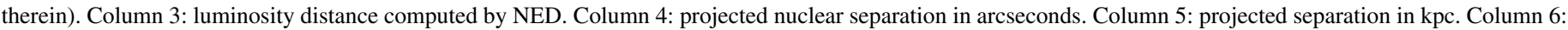

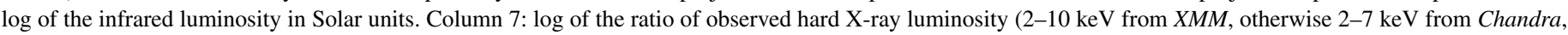

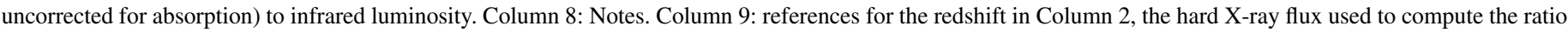

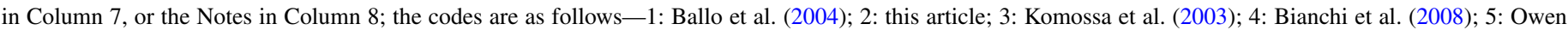

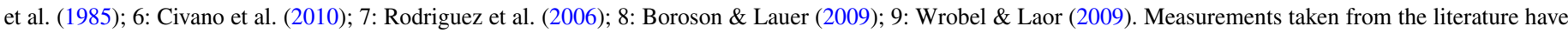

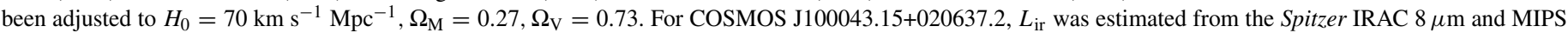
$24 \mu \mathrm{m}$ measurements in Civano et al. (2010) utilizing Equation (1) of the current study.

radio cores contain AGNs orbiting with a rotational period of $1.5 \times 10^{5} \mathrm{yr}$ within a single elliptical galaxy host. It is notable that the sum of the SMBH masses in $4 \mathrm{C}+37.11, \sim 1.5 \times 10^{8} \mathrm{M}_{\odot}$, is similar to Mrk 266; this implies that LIRGs with bulge masses similar to the companions in Mrk 266 may end up as remnants similar to $4 \mathrm{C}+37.11$. The soft X-ray luminosity of $4 \mathrm{C}+37.11$ is $\approx 170$ times larger than Mrk 266. (Hard X-ray observations of $4 \mathrm{C}+37.11$ are not available.)

Very few additional binary AGNs have been confirmed, although there are a few candidates. Observations of double-peaked emission lines in the radio-quiet QSO SDSS $\mathrm{J} 153636.22+044127.0(z=0.388)$ have been interpreted as a candidate binary SMBH with masses $8 \times 10^{8}$ and $2 \times 10^{7}$ inferred to be separated by only 0.02 mas $(0.1 \mathrm{pc})$ (Boroson \& Lauer 2009). Wrobel \& Laor (2009) reported detection of two $8.5 \mathrm{GHz}$ radio continuum sources separated by 0. .97 $(5.1 \mathrm{kpc})$ within the aperture of the original spectroscopic observations, suggesting this is another kiloparsec-scale dual AGN system. However, further observations show that the second radio source is an elliptical galaxy that is not the origin of the redshifted or blueshifted Balmer lines (Lauer \& Boroson 2009). Another possibility is that SDSS J153636.22+044127.0 is similar to other "double-peak" AGNs such as 3C 390.3 and Arp 102B, which were also promoted as candidate binary AGNs but later found to be more consistent with a rapidly rotating accretion disk within a single broadline region (Gaskell 2010). Additional observations are needed to distinguish between these alternate hypotheses.

Table 10 lists basic parameters for the small number of confirmed dual AGNs and putative binary AGNs discussed above. Due to the high degree of uncertainty in age dating mergers with factors such as nuclear separation, the morphology of tidal debris, and varying techniques applied in the simulations of specific systems, we propose a merger sequence based on the quantitative parameter $L_{h x} / L_{\text {ir }}$, where $L_{h x}$ is computed using the observed flux (uncorrected for obscuration) in the $2-10 \mathrm{keV}$ hard $\mathrm{X}$-ray band from $X M M$ where available, otherwise using the $2-7 \mathrm{keV}$ band from Chandra. According to the evolutionary paradigm whereby ULIRGs transform into UV/optical QSOs during the merger of gas-rich galaxies (Sanders et al. 1988a, 1988b), the degree to which the luminous optical/UV/X-ray emission from the accretion disk is obscured should diminish as superwinds driven by star formation and/or AGNs diffuse or expel the dust from the system. To the extent that increasing $\log \left(L_{h x} / L_{\mathrm{ir}}\right)$, given in parenthesis below, is a reliable gauge of the degree to which the embedded AGNpowered UV/X-ray sources have emerged from their obscuring cocoons, a presumptive sequence is: Mrk $171(-4.1) \rightarrow$ Mrk $266(-3.5) \rightarrow$ NGC $6240(-3.1) \rightarrow$ Mrk $463(-2.8) \rightarrow$ COSMOS J100043.15 + $020637.2(-1.7) .^{35}$

Despite the growing number of kiloparsec-scale dual AGNs, the number of sub-parsec binary AGNs in the class of $4 \mathrm{C}+37.11$ remains very small. Currently SDSS J153636.22+044127.0 is the only candidate out of $\approx 17,500$ AGNs with $z<0.70$ in the SDSS (Lauer \& Boroson 2009). Similarly, a recent study of Very Long Baseline Array data for over 3100 radioluminous AGNs found only one binary, the previously known 4C +37.11 (Burke-Spolaor 2011). Since major mergers provide a natural process to form binary SMBHs, the apparent dearth of binary AGNs needs explanation. Gaskell (2010) has noted two possibilities: either the lifetime of binary SMBHs is much shorter than predicted (i.e., they inspiral very rapidly rather than remaining in a stalled orbit) or fueling of the accretion $\operatorname{disk}(\mathrm{s})$ is somehow suspended. The former explanation seems unlikely because the very presence of the accretion disks forms a significant buffer against rapid inspiral and effectively "softens" the SMBH merger process. A possible physical mechanism for the latter suggestion may be that AGN jets have the potential to impart enough energy into the inner parsecs of a galaxy to impede gas inflow to the accretion disk (Nagar et al. 2005). We propose another physically viable alternative. If dual AGNs are confirmed in many more (U)LIRGs in intermediate stages of the merger process with kpc scale separations (i.e., if most of the LINERs in the GOALS and IRAS 1 Jy ULIRG samples are confirmed to be ionized by a power-law source as suggested by the radio observations of Nagar et al. 2003), this raises the possibility that there will be an insufficient fuel supply remaining to power luminous binary AGNs late in the merger process.

\footnotetext{
35 SDSS J153636.22+044127.0 is omitted from the proposed sequence because its nature as a true binary AGN is controversial, and the required IR and X-ray measurements are not yet available.
} 
The lifetime of nuclear starbursts and AGNs is inversely proportional to the efficiency of gas depletion and ranges between $\sim 10^{7}$ for ULIRGs and $\sim 10^{8}$ yr for LIRGs (e.g., Solomon \& Vanden Bout 2005). The lifetime of powerful radioloud AGNs has also been estimated to be only $\sim 10^{7} \mathrm{yr}$ (Bird et al. 2008). This is similar to the dynamical timescale of the final coalescence of the nuclei with high circular velocity at the core of a merger, but only $1 \%-10 \%$ of the $\sim 10^{9}$ Gyr timescale for dynamical relaxation of the stars in outer orbits of the remnant galaxy (e.g., Mihos 1999). Therefore, it is possible that starbursts and dual AGNs may consume or expel the bulk of available gas relatively early in many major mergers, leaving insufficient fuel to power QSOs that can be detected spectroscopically through the stage when the SMBHs inspiral to form a true binary and then coalesce or recoil. This hypothesis counters simulations that find AGN accretion rates peak during the SMBH coalescence (e.g., Johansson et al. 2009).

\section{SUMMARY AND CONCLUSIONS}

We have presented the results of a multi-wavelength campaign on the LIRG system Markarian 266 that has yielded the following conclusions regarding the morphology, nuclei, dust, molecular gas, outflows, star clusters, and radiation between the nuclei, as well as implications for a better understanding of the origin of ULIRGs and binary AGNs.

1. HST images show that the "double-nucleus galaxy" observed in low-resolution images is a strongly interacting pair; we classify the $\mathrm{SW}$ and $\mathrm{NE}$ galaxies as $\mathrm{SBb}$ (pec) and S0/a (pec), respectively. Deeper imaging reveals asymmetric tidal debris spanning $\approx 100 \mathrm{kpc}$. At $H$ band, the companions are more luminous than $L^{*}$ galaxies, and their estimated stellar masses are $6.1 \times 10^{10} M_{\odot}(\mathrm{SW})$ and $4.4 \times 10^{10} M_{\odot}(\mathrm{NE})$. The stellar bulge luminosities imply remarkably similar nuclear black hole masses of $2 \times 10^{8} \mathrm{M}_{\odot}$.

2. The NE (LINER) nucleus is emitting 6.4 times more hard X-ray (2-7 keV) flux than the SW (Seyfert 2) nucleus, yet the luminosity of the NE galaxy is only $\approx 20 \%$ of the SW galaxy at $24 \mu \mathrm{m}$ and in CO (1-0) molecular gas emission. The Chandra spectra indicate that the SW nucleus is likely the primary source of a bright $\mathrm{Fe} \mathrm{K} \alpha$ line detected in the $X M M$ spectrum of the total system, consistent with a reflection-dominated X-ray spectrum of a heavily obscured AGN. The $\mathrm{F}(\mathrm{X}$-ray)/F([O III $]$ ) flux ratios also indicate that the SW nucleus is much more heavily obscured than the NE nucleus.

3. Soft $(0.4-2 \mathrm{keV}) \mathrm{X}$-ray emission extends $15 \mathrm{kpc}$ to the north of the system, between the nuclei, and $3 \mathrm{kpc}$ west of the SW nucleus. The smoothed X-ray structure corresponds remarkably well with filaments in $\mathrm{H} \alpha$ and [O III] images. A ridge of X-ray emission between the galaxies has an X-ray spectrum consistent with $T \sim 10^{7} \mathrm{~K}$ shockheated gas, strengthening the idea that the corresponding radio emission is shock-induced synchrotron radiation. The lack of detected $9.7 \mu \mathrm{m} \mathrm{H}_{2} \mathrm{~S}(3)$ line emission supports the suggestion that a very fast shock at the interface of superwinds expanding from the two galaxies might generate the observed non-thermal radio continuum emission and heat the gas above the dissociation temperature of $\mathrm{H}_{2}$. The derived cooling time of the $\mathrm{X}$-ray emitting gas between the nuclei is only $\approx 4 \mathrm{Myr}$.

4. The HST optical images reveal a circumnuclear arc containing three knots 240 pc west of the NE nucleus. The arc is embedded within $B$-band emission with a filamentary, bi-conic morphology extending over a radius of $1.2 \mathrm{kpc}$. This region appears to be dominated by nebular emission from the narrow-line region of a double-sided AGN ionization cone. Radio continuum emission at $1.6 \mathrm{GHz}$ peaks at the optical/near-IR nucleus and has components in alignment with the nucleus and the circumnuclear $B$-band knots $\left(\right.$ P.A. $\left.=56^{\circ}\right)$. A plausible explanation is that the optical knots are clouds entrained in a shock front produced by an AGN-powered collimated plasma outflow (jet). We liken this region to the radiative bow shock $230 \mathrm{pc}$ south of the LINER in M51.

5. Mid-IR spectral diagnostics suggest that the bolometric luminosity is powered by roughly an equal mixture of radiation from AGNs and star formation, with substantial scatter among the various methods. Newly constructed SEDs give infrared luminosities of $L_{\text {ir }}=(2.3,0.7,3.4) \times 10^{11} L_{\odot}$ for the SW, NE, and total system, respectively. Decomposition of the SED of the total system indicates that cold $(26 \mathrm{~K})$, cool $(72 \mathrm{~K})$, warm $(235 \mathrm{~K})$, and hot $(\sim 1500 \mathrm{~K})$ thermal dust components contribute approximately $57 \%, 20 \%, 12 \%$, and $11 \%$ of the bolometric luminosity, respectively. The total cold dust mass estimate is $1.5( \pm 0.4) \times 10^{8} M_{\odot}$.

6. HST imaging resolves the Northern Loop extending 6-20 kpc from the galaxies into a fragmented morphology suggestive of Rayleigh-Taylor instabilities. A few structures $300-600 \mathrm{pc}$ in length are radially aligned with the NE nucleus, suggestive of bow shocks predicted by hydrodynamic simulations of galactic superwinds. Detection of $24 \mu \mathrm{m}$ emission in the Northern Loop implies that at least $\sim 2 \times 10^{7} M_{\odot}$ of dust is being swept out of the system by the superwind during a "blow-out" phase that is well underway prior to the final galaxy merger.

7. Approximately 120 star clusters detected with HST are concentrated in the SW galaxy and at the base of the Northern Loop; most have estimated ages less than $50 \mathrm{Myr}$ and masses of $\sim 10^{5} M_{\odot}$. The ratios of cluster surface densities, $L_{\mathrm{CO}}$, and $L_{\mathrm{ir}}$ within $3 \mathrm{kpc}$ of each nucleus are similar (i.e., $\mathrm{L}(\mathrm{SW}) / \mathrm{L}(\mathrm{NW}) \approx 4-5)$. The star cluster luminosity function is similar to what has been found in other LIRGs, and the unobscured clusters contribute little to powering the total infrared luminosity.

8. We conclude that Mrk $266 \mathrm{NE}$ is an AGN based on the presence of: (1) an obscured, hard X-ray point source, (2) a radiative bow shock aligned with ionization cones and apparent radio plasma outflow, (3) PAH emission with small equivalent widths and a deficiency of 6.2 and $7.7 \mu \mathrm{m}$ flux relative to $11.3 \mu \mathrm{m}$ flux, and (4) an $\mathrm{H}_{2}(1-0) \mathrm{S}(1)$ to $\mathrm{Br} \gamma$ flux ratio of 3.5 . The spatially extended $\mathrm{H}_{2}$ emission demonstrates that LINERs can exhibit strong signatures of an AGN and shock excitation.

9. The bulk of the CO (1-0) emission in the system is aligned with the major axis of the SW galaxy. HCN (1-0) is aligned with $\mathrm{H}_{2} \mathrm{~S}(3) 9.7 \mu \mathrm{m}$ emission in the nucleus of Mrk 266 NE. However, the CO (1-0) and HCO + (1-0) emission peaks are located $\sim 1 \mathrm{kpc} \mathrm{SE}$ of the NE nucleus, suggesting that either most of the cold molecular gas has already been stripped from the NE galaxy or gas is being transferred from the SW galaxy to the NE galaxy. Approximately $40 \%$ of the total CO (1-0) emission is bridging the galaxies, likely infalling toward the center of mass or transferring between the galaxies. In this regard, 
Mrk 266 appears to be in an evolutionary stage similar to VV 114, NGC 6090, and NGC 6240.

10. Two lines of evidence suggest Mrk 266 may evolve into a ULIRG. First, in the context of merger simulations, the galaxies lie within an asymmetric, low surface brightness halo of tidal debris spanning $\approx 100 \mathrm{kpc}$, with a projected separation of $6 \mathrm{kpc}$ and a relative velocity of only $135 \mathrm{~km} \mathrm{~s}^{-1}$, indicating they are likely undergoing their second or third encounter with only 50 to 250 Myr remaining until they merge via tidal dissipation. Therefore, Mrk 266 appears to be in a short-lived stage when the nuclei are about to enter the final phase of coalescence characteristic of ULIRGs. Second, the total cold molecular gas mass of $\approx 7 \times 10^{9} M_{\odot}$ is similar to local and high-redshift ULIRGs. Since $\approx 40 \%$ of the total CO (1-0) emission is located between the galaxies, this reservoir is available to form more stars and to fuel the AGNs as the stellar systems and nuclei inexorably coalesce.

11. We propose that Mrk 266 belongs to an evolutionary sequence in which dual AGNs with kiloparsec separations represent precursors to putative binary $A G N s$ with parsec-scale orbital radii. In this scenario, where Mrk 266 is in an intermediate phase between Mrk 171 and NGC 6240, the global $L_{x} / L_{\text {ir }}$ ratio increases by over two orders of magnitude as obscuring material is expelled by outflows to gradually expose previously obscured AGNs.

12. Since major mergers provide a natural process to form SMBH/AGN pairs, the scarcity of confirmed and candidate binary QSOs in large spectroscopic and VLBI surveys is unexpected. While two possibilities are raised in the literature, that either the SMBHs inspiral very rapidly or fueling of the accretion disks is quenched during the binary phase, we propose a third hypothesis. Since the gasdepletion lifetime of ULIRGs and LIRGs is 10-100 times shorter than the timescale for creation of a binary SMBH ( $\gtrsim 1 \mathrm{Gyr}$ ), it is possible that, in most instances, the gas will be consumed by star formation and accretion during a dual AGN phase long before the SMBHs inspiral to sub-parsec separation.

This work is based on observations with the following facilities: the Spitzer Space Telescope, which is operated by the Jet Propulsion Laboratory, California Institute of Technology under a contract with NASA, the NASA/ESA Hubble Space Telescope, which is operated by the Association of Universities for Research in Astronomy, Inc. under NASA contract NAS526555, the Chandra X-ray Observatory Center, which is operated by the Smithsonian Astrophysical Observatory for and on behalf of NASA under contract NAS8-03060, and the Galaxy Evolution Explorer (GALEX), which is operated for NASA by the California Institute of Technology under NASA contract NAS5-98034. The Aladin (CDS, Strasbourg) and SAOImage $D S 9$ tools were used for image analysis. Support for this work was provided by the following NASA grants: an award issued by JPL/Caltech (Spitzer PID 3672, PI: J. Mazzarella); HST-GO10592.01-A (ACS imaging, PI: A. Evans) and program 11235 (NICMOS imaging, PI: J. Surace) issued by the STScI. K. Iwasawa acknowledges support from Spanish grant MICINN (AYA2010-21782-C03-01). This research made use of the NASA/IPAC Extragalactic Database (NED) and the Infrared Science Archive (IRSA), which are operated by the Jet Propulsion Laboratory, California Institute of Technology, un- der contract with NASA. We thank Masa Imanishi for providing FITS files for the Nobeyama Millimeter Array observations and Tsuyoshi Ishigaki for supplying optical emission-line images. We thank Chris Mihos for helpful discussions regarding merger timescales. Finally, we thank the anonymous referee for very helpful comments that led to improvements to the manuscript.

Facilities: CXO (ACIS-S), XMM (EPIC pn, OM), GALEX (FUV, NUV), HST (ACS, NICMOS), UH:2.2m, Spitzer (IRAC, MIPS, IRS)

Objects: Mrk 266, Mrk 266 SW, Mrk 266 NE

\section{APPENDIX}

\section{A.1. Photometric Data for Mrk 266}

Figure 31 displays regions parameterized in Table 11 that were used to perform aperture photometry tabulated in Tables 12 and 13, as plotted in Figure 9 (Section 2.6.2).

\section{A.2. Clarification of Regions Covered in Published Spitzer IRS Data for Mrk 266}

As noted in Section 2.7, mid-IR spectroscopy of Mrk 266 obtained in staring mode with the Spitzer IRS is complicated due to aperture affects. To facilitate interpretation of the IRS spectroscopy published to date, the available observations are summarized here, and Table 14 provides clarification of which emission features have been measured for each component of the system.

In the low spectral resolution (R 65-130) observations (AOR 3755264; GTO program P00014; PI: J. Houck), the Short-Low (SL, 5.2-14.5 $\mu \mathrm{m}$ ) aperture of the IRS contained Mrk $266 \mathrm{NE}$ (off center), and missed both Mrk $266 \mathrm{SW}$ and the central radio/X-ray source; the Long-Low (LL, 14-38 $\mu \mathrm{m}$ ) slit contained Mrk $266 \mathrm{SW}$ and the radio/X-ray source between the nuclei, but it missed Mrk 266 NE. Neither aperture had the

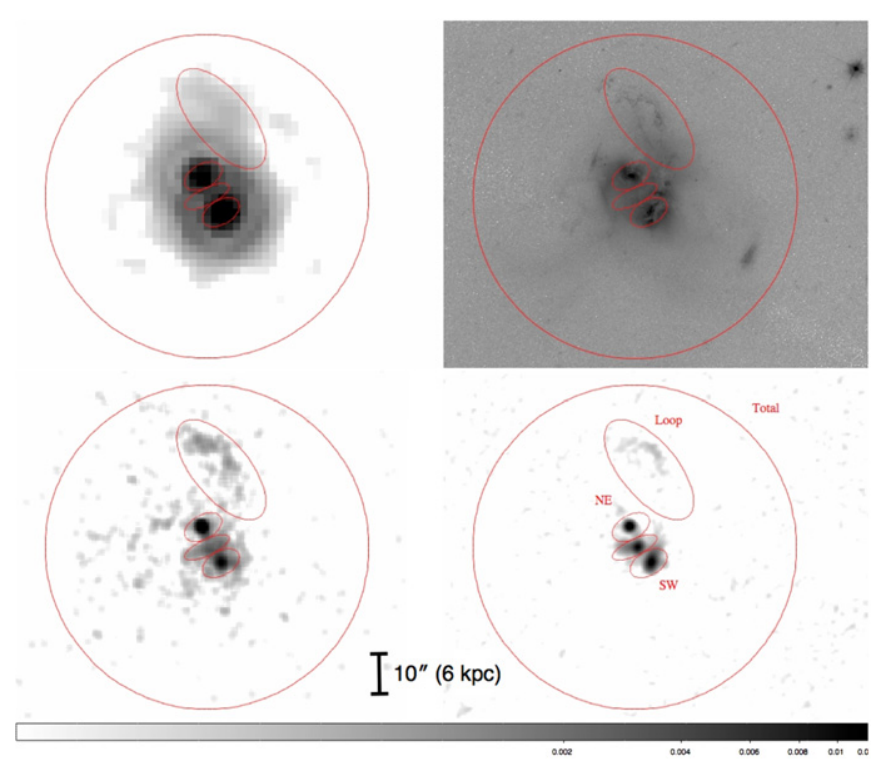

Figure 31. Regions used to perform aperture photometry of the nuclei, central region, and Northern Loop superposed on the $24 \mu \mathrm{m}$ Spitzer MIPS image (upper left), the HST ACS B-band image (upper right), the Chandra full-band X-ray image (lower left), and the $20 \mathrm{~cm}$ radio continuum image (lower right). All four images are shown at the same scale and orientation.

(A color version of this figure is available in the online journal.) 
Table 11

Elliptical Apertures for Regions of Interest

\begin{tabular}{lccrr}
\hline \hline $\begin{array}{l}\text { Region } \\
(1)\end{array}$ & $\begin{array}{c}a^{\prime \prime} \\
(2)\end{array}$ & \multicolumn{1}{c}{$\begin{array}{c}b^{\prime \prime} \\
(4)\end{array}$} & $\begin{array}{c}\text { P.A. } \\
(5)\end{array}$ \\
\hline SW & $(13: 38: 17.28,+48: 16: 32.1)$ & 5.0 & 3.0 & 120 \\
NE & $(13: 38: 17.79,+48: 16: 41.2)$ & 5.0 & 3.0 & 120 \\
Between galaxies & $(13: 38: 17.51,+48: 16: 36.8)$ & 6.0 & 2.0 & 115 \\
Northern Loop & $(13: 38: 17.54,+48: 16: 56.0)$ & 17.0 & 7.5 & 40 \\
Total System & $(13: 38: 17.66,+48: 16: 35.8)$ & 40.0 & 40.0 & $\ldots$ \\
\hline
\end{tabular}

Notes. Parameters for elliptical apertures used for photometric measurements, as illustrated in Figure 31.

Table 12

Photometric Data for Mrk 266-Total System

\begin{tabular}{|c|c|c|c|c|}
\hline Bandpass & $\begin{array}{c}v \\
(\mathrm{~Hz}) \\
(2)\end{array}$ & $\begin{array}{c}f_{v} \\
(\mathrm{Jy}) \\
(3)\end{array}$ & $\begin{array}{c}\sigma_{f_{v}} \\
(\mathrm{Jy}) \\
(4)\end{array}$ & References \\
\hline $2-10 \mathrm{keV}$ (ASCA) & $1.45 \mathrm{E}+18$ & $2.48 \mathrm{E}-08$ & 5.0E-09 & 2 \\
\hline $2.0-7.0 \mathrm{keV}(X M M)$ & $1.09 \mathrm{E}+18$ & $2.11 \mathrm{E}-08$ & $6.3 \mathrm{E}-09$ & 1 \\
\hline $0.7-7 \mathrm{keV}$ (ASCA) & $9.31 \mathrm{E}+17$ & $3.65 \mathrm{E}-08$ & 7.3E-09 & 2 \\
\hline $0.5-2.0 \mathrm{keV}(X M M)$ & $3.02 \mathrm{E}+17$ & $5.96 \mathrm{E}-08$ & $1.2 \mathrm{E}-08$ & 1 \\
\hline GALEX FUV & $1.96 \mathrm{E}+15$ & 7.94E-04 & $3.1 \mathrm{E}-05$ & 1 \\
\hline$X M M$-OM UVW2 & $1.41 \mathrm{E}+15$ & $2.06 \mathrm{E}-03$ & 8.0E-04 & 1 \\
\hline GALEX NUV & $1.32 \mathrm{E}+15$ & $1.63 \mathrm{E}-03$ & $3.4 \mathrm{E}-05$ & 1 \\
\hline$X M M-O M$ UVW1 & $1.03 \mathrm{E}+15$ & $2.12 \mathrm{E}-03$ & $2.6 \mathrm{E}-04$ & 1 \\
\hline$X M M-\mathrm{OM} \mathrm{U}$ & $8.71 \mathrm{E}+14$ & $3.87 \mathrm{E}-03$ & $1.4 \mathrm{E}-03$ & 1 \\
\hline$H S T$ ACS F435W (B) & $6.89 \mathrm{E}+14$ & 8.92E-03 & 4.1E-05 & 1 \\
\hline$V$ (Johnson) & $5.42 \mathrm{E}+14$ & $1.82 \mathrm{E}-02$ & $8.6 \mathrm{E}-04$ & 3 \\
\hline LW2 (ISOCAM) & $4.44 \mathrm{E}+13$ & $1.14 \mathrm{E}-01$ & $1.4 \mathrm{E}-02$ & 4 \\
\hline$R$ (Johnson) & $4.28 \mathrm{E}+14$ & $2.86 \mathrm{E}-02$ & 8.0E-04 & 3 \\
\hline HST ACS F814W (I) & $3.68 \mathrm{E}+14$ & $2.97 \mathrm{E}-02$ & 5.5E-05 & 1 \\
\hline 2MASS J $(1.2 \mu \mathrm{m})$ & $2.43 \mathrm{E}+14$ & $5.44 \mathrm{E}-02$ & $6.5 \mathrm{E}-04$ & 1 \\
\hline 2MASS H $(1.6 \mu \mathrm{m})$ & $1.80 \mathrm{E}+14$ & $6.42 \mathrm{E}-02$ & $8.8 \mathrm{E}-04$ & 1 \\
\hline 2MASS Ks $(2.2 \mu \mathrm{m})$ & $1.39 \mathrm{E}+14$ & $6.02 \mathrm{E}-02$ & 8.3E-04 & 1 \\
\hline Spitzer $3.6 \mu \mathrm{m}$ & $8.44 \mathrm{E}+13$ & $3.82 \mathrm{E}-02$ & $5.7 \mathrm{E}-03$ & 1 \\
\hline Spitzer $4.5 \mu \mathrm{m}$ & $6.67 \mathrm{E}+13$ & $3.05 \mathrm{E}-02$ & 4.6E-03 & 1 \\
\hline Spitzer $5.8 \mu \mathrm{m}$ & $5.23 \mathrm{E}+13$ & $6.80 \mathrm{E}-02$ & $1.0 \mathrm{E}-02$ & 1 \\
\hline Spitzer $8.0 \mu \mathrm{m}$ & $3.81 \mathrm{E}+13$ & $1.79 \mathrm{E}-01$ & 2.7E-02 & 1 \\
\hline IRAS $12 \mu \mathrm{m}$ & $2.50 \mathrm{E}+13$ & $3.20 \mathrm{E}-01$ & $1.8 \mathrm{E}-02$ & 5 \\
\hline Spitzer $24 \mu \mathrm{m}$ & $1.27 \mathrm{E}+13$ & $9.55 \mathrm{E}-01$ & $1.9 \mathrm{E}-01$ & 1 \\
\hline$I R A S 25 \mu \mathrm{m}$ & $1.20 \mathrm{E}+13$ & $1.07 \mathrm{E}+00$ & $2.8 \mathrm{E}-02$ & 5 \\
\hline$I R A S 60 \mu \mathrm{m}$ & $5.00 \mathrm{E}+12$ & $7.25 \mathrm{E}+00$ & 3.3E-02 & 5 \\
\hline Spitzer $70 \mu \mathrm{m}$ & 4.20E+12 & $8.95 \mathrm{E}+00$ & $1.8 \mathrm{E}+00$ & 1 \\
\hline IRAS $100 \mu \mathrm{m}$ & $3.00 \mathrm{E}+12$ & $1.01 \mathrm{E}+01$ & $1.4 \mathrm{E}-01$ & 5 \\
\hline Spitzer $160 \mu \mathrm{m}$ & $1.92 \mathrm{E}+12$ & $6.41 \mathrm{E}+00$ & $1.3 \mathrm{E}+00$ & 1 \\
\hline $170 \mu \mathrm{m}(I S O)$ & $1.76 \mathrm{E}+12$ & $8.73 \mathrm{E}+00$ & $2.6 \mathrm{E}+00$ & 6 \\
\hline SCUBA $850 \mu \mathrm{m}$ & $3.53 \mathrm{E}+11$ & $8.20 \mathrm{E}-02$ & $1.7 \mathrm{E}-02$ & 7 \\
\hline $20.0 \mathrm{GHz}(\mathrm{OVRO})$ & $2.00 \mathrm{E}+10$ & $1.49 \mathrm{E}-02$ & $1.3 \mathrm{E}-03$ & 8 \\
\hline $4.85 \mathrm{GHz}$ & $4.85 \mathrm{E}+09$ & $4.70 \mathrm{E}-02$ & 7.1E-03 & 9 \\
\hline $1.46 \mathrm{GHz}$ (VLA) & $1.46 \mathrm{E}+09$ & $1.07 \mathrm{E}-01$ & $6.2 \mathrm{E}-03$ & 8 \\
\hline Texas $365 \mathrm{MHz}$ & $3.65 \mathrm{E}+08$ & $3.53 \mathrm{E}-01$ & 4.3E-02 & 10 \\
\hline $151 \mathrm{MHz}(6 \mathrm{C})$ & $1.52 \mathrm{E}+08$ & $5.90 \mathrm{E}-01$ & 4.0E-02 & 11 \\
\hline
\end{tabular}

Notes. Reference codes in Column (5): $1=$ New measurements in this article; $2=$ Ueda et al. (2005); 3 = Huchra (1977); 4 = Ramos Almeida et al. (2007); $5=$ Sanders et al. (2003); $6=$ Stickel et al. (2004); $7=$ Dunne et al. (2000); $8=$ Edelson (1987); $9=$ Becker et al. (1991); $10=$ Douglas et al. (1996); $11=$ Hales et al. (1990).

orientation required to sample the Northern Loop. The lowresolution IRS spectrum displayed in Brandl et al. (2006) is therefore a mixture of emission from Mrk $266 \mathrm{NE}$ in the 5-14 $\mu \mathrm{m}$ region with emission from Mrk $266 \mathrm{SW}$ (and the radio/X-ray source between the nuclei) in the $14-38 \mu \mathrm{m}$ region.
Table 13

Photometric Data for Mrk 266-Components

\begin{tabular}{|c|c|c|c|c|}
\hline Bandpass & $\begin{array}{c}v \\
(\mathrm{~Hz}) \\
(2)\end{array}$ & $\begin{array}{c}f_{v} \\
(\mathrm{Jy}) \\
(3)\end{array}$ & $\begin{array}{c}\sigma_{f_{v}} \\
(\mathrm{Jy}) \\
(4)\end{array}$ & References \\
\hline \multicolumn{5}{|c|}{ Mrk 266 SW } \\
\hline $2.0-7.0 \mathrm{keV}$ & $1.09 \mathrm{E}+18$ & 2.02E-09 & $6.1 \mathrm{E}-10$ & 1 \\
\hline $0.5-2.0 \mathrm{keV}$ & $3.02 \mathrm{E}+17$ & 7.28E-09 & $1.5 \mathrm{E}-09$ & 1 \\
\hline$X M M-O M$ UVW2 & $1.41 \mathrm{E}+15$ & $1.68 \mathrm{E}-04$ & $1.1 \mathrm{E}-05$ & 1 \\
\hline$X M M-\mathrm{OM}$ UVW1 & $1.03 \mathrm{E}+15$ & $2.72 \mathrm{E}-04$ & 5.4E-05 & 1 \\
\hline$X M M-\mathrm{OM} \mathrm{U}$ & $8.71 \mathrm{E}+14$ & $5.12 \mathrm{E}-04$ & $2.2 \mathrm{E}-05$ & 1 \\
\hline HST ACS F435W (B) & $6.89 \mathrm{E}+14$ & $1.31 \mathrm{E}-03$ & 1.2E-05 & 1 \\
\hline HST ACS F814W (I) & $3.68 \mathrm{E}+14$ & $5.03 \mathrm{E}-03$ & 2.3E-05 & 1 \\
\hline 2MASS J $(1.2 \mu \mathrm{m})$ & $2.43 \mathrm{E}+14$ & $1.04 \mathrm{E}-02$ & $2.5 \mathrm{E}-04$ & 1 \\
\hline NICMOS H $(1.6 \mu \mathrm{m})$ & $1.87 \mathrm{E}+14$ & $1.45 \mathrm{E}-02$ & 1.7E-04 & 1 \\
\hline 2MASS H $(1.6 \mu \mathrm{m})$ & $1.80 \mathrm{E}+14$ & $1.46 \mathrm{E}-02$ & 3.3E-04 & 1 \\
\hline 2MASS Ks $(2.2 \mu \mathrm{m})$ & $1.39 \mathrm{E}+14$ & $1.53 \mathrm{E}-02$ & $3.1 \mathrm{E}-04$ & 1 \\
\hline Spitzer $3.6 \mu \mathrm{m}$ & $8.44 \mathrm{E}+13$ & $1.50 \mathrm{E}-02$ & 2.3E-03 & 1 \\
\hline Spitzer $4.5 \mu \mathrm{m}$ & $6.67 \mathrm{E}+13$ & $1.47 \mathrm{E}-02$ & $2.2 \mathrm{E}-03$ & 1 \\
\hline Spitzer $5.8 \mu \mathrm{m}$ & $5.23 \mathrm{E}+13$ & $4.59 \mathrm{E}-02$ & $6.9 \mathrm{E}-03$ & 1 \\
\hline Spitzer $8.0 \mu \mathrm{m}$ & $3.81 \mathrm{E}+13$ & $1.28 \mathrm{E}-01$ & $1.9 \mathrm{E}-02$ & 1 \\
\hline Spitzer $24 \mu \mathrm{m}$ & $1.27 \mathrm{E}+13$ & $5.91 \mathrm{E}-01$ & $1.2 \mathrm{E}-01$ & 1 \\
\hline Spitzer $70 \mu \mathrm{m}^{\mathrm{a}}$ & $4.20 \mathrm{E}+12$ & $6.01 \mathrm{E}+00$ & $1.2 \mathrm{E}+00$ & 1 \\
\hline Spitzer $160 \mu \mathrm{m}^{\mathrm{a}}$ & $1.92 \mathrm{E}+12$ & $4.30 \mathrm{E}+00$ & 8.6E-01 & 1 \\
\hline $2 \mathrm{~cm}$ & $1.50 \mathrm{E}+10$ & $4.90 \mathrm{E}-03$ & 7.0E-04 & 12 \\
\hline $6 \mathrm{~cm}$ & $4.89 \mathrm{E}+09$ & $1.01 \mathrm{E}-02$ & $5.0 \mathrm{E}-04$ & 12 \\
\hline $20 \mathrm{~cm}$ & $1.47 \mathrm{E}+09$ & $3.24 \mathrm{E}-02$ & $1.4 \mathrm{E}-03$ & 12 \\
\hline \multicolumn{5}{|c|}{ Mrk $266 \mathrm{NE}$} \\
\hline $2.0-7.0 \mathrm{keV}$ & $1.09 \mathrm{E}+18$ & $1.29 \mathrm{E}-08$ & 3.9E-09 & 1 \\
\hline $0.5-2.0 \mathrm{keV}$ & $3.02 \mathrm{E}+17$ & 9.93E-09 & 2.0E-09 & 1 \\
\hline$X M M$-OM UVW2 & $1.41 \mathrm{E}+15$ & $2.45 \mathrm{E}-04$ & $2.0 \mathrm{E}-05$ & 1 \\
\hline$X M M-\mathrm{OM}$ UVW1 & $1.03 \mathrm{E}+15$ & $3.63 \mathrm{E}-04$ & $9.1 \mathrm{E}-05$ & 1 \\
\hline$X M M-\mathrm{OM} \mathrm{U}$ & $8.71 \mathrm{E}+14$ & $6.49 \mathrm{E}-04$ & $3.0 \mathrm{E}-05$ & 1 \\
\hline$H S T$ ACS F435W $(B)$ & $6.89 \mathrm{E}+14$ & $1.59 \mathrm{E}-03$ & $1.5 \mathrm{E}-05$ & 1 \\
\hline HST ACS F814W (I) & $3.68 \mathrm{E}+14$ & $6.02 \mathrm{E}-03$ & 2.8E-05 & 1 \\
\hline 2MASS J $(1.2 \mu \mathrm{m})$ & $2.43 \mathrm{E}+14$ & $9.62 \mathrm{E}-03$ & 2.4E-04 & 1 \\
\hline NICMOS H $(1.6 \mu \mathrm{m})$ & $1.87 \mathrm{E}+14$ & $1.22 \mathrm{E}-02$ & $1.6 \mathrm{E}-04$ & 1 \\
\hline 2MASS H $(1.6 \mu \mathrm{m})$ & $1.80 \mathrm{E}+14$ & $1.22 \mathrm{E}-02$ & $3.0 \mathrm{E}-04$ & 1 \\
\hline 2MASS Ks $(2.2 \mu \mathrm{m})$ & $1.39 \mathrm{E}+14$ & $1.09 \mathrm{E}-02$ & 2.6E-04 & 1 \\
\hline Spitzer $3.6 \mu \mathrm{m}$ & $8.44 \mathrm{E}+13$ & 7.35E-03 & $1.1 \mathrm{E}-03$ & 1 \\
\hline Spitzer $4.5 \mu \mathrm{m}$ & $6.67 \mathrm{E}+13$ & $5.90 \mathrm{E}-03$ & 8.9E-04 & 1 \\
\hline Spitzer $5.8 \mu \mathrm{m}$ & $5.23 \mathrm{E}+13$ & $9.57 \mathrm{E}-03$ & $1.4 \mathrm{E}-03$ & 1 \\
\hline Spitzer $8.0 \mu \mathrm{m}$ & $3.81 \mathrm{E}+13$ & $2.23 \mathrm{E}-02$ & $3.3 \mathrm{E}-03$ & 1 \\
\hline Spitzer $24 \mu \mathrm{m}$ & $1.27 \mathrm{E}+13$ & $3.28 \mathrm{E}-01$ & $6.6 \mathrm{E}-02$ & 1 \\
\hline Spitzer $70 \mu \mathrm{m}^{\mathrm{a}}$ & $4.20 \mathrm{E}+12$ & $1.74 \mathrm{E}+00$ & 7.0E-01 & 1 \\
\hline Spitzer $160 \mu \mathrm{m}^{\mathrm{a}}$ & $1.92 \mathrm{E}+12$ & $1.25 \mathrm{E}+00$ & $5.0 \mathrm{E}-01$ & 1 \\
\hline $2 \mathrm{~cm}$ & $1.50 \mathrm{E}+10$ & 4.10E-03 & $6.0 \mathrm{E}-04$ & 12 \\
\hline $6 \mathrm{~cm}$ & $4.89 \mathrm{E}+09$ & $1.22 \mathrm{E}-02$ & 4.0E-04 & 12 \\
\hline $20 \mathrm{~cm}$ & $1.47 \mathrm{E}+09$ & $2.35 \mathrm{E}-02$ & $1.0 \mathrm{E}-03$ & 12 \\
\hline \multicolumn{5}{|c|}{ Between the nuclei } \\
\hline $2.0-7.0 \mathrm{keV}$ & $1.09 \mathrm{E}+18$ & $9.19 \mathrm{E}-10$ & $2.8 \mathrm{E}-10$ & 1 \\
\hline $0.5-2.0 \mathrm{keV}$ & $3.02 \mathrm{E}+17$ & 8.60E-09 & 1.7E-09 & 1 \\
\hline $2 \mathrm{~cm}$ & $1.50 \mathrm{E}+10$ & $2.70 \mathrm{E}-03$ & $5.0 \mathrm{E}-04$ & 12 \\
\hline $6 \mathrm{~cm}$ & $4.89 \mathrm{E}+09$ & $6.00 \mathrm{E}-03$ & 2.7E-03 & 12 \\
\hline $20 \mathrm{~cm}$ & $1.47 \mathrm{E}+09$ & $2.15 \mathrm{E}-02$ & 9.0E-04 & 12 \\
\hline \multicolumn{5}{|c|}{ Northern loop } \\
\hline $0.5-2.0 \mathrm{keV}$ & $3.02 \mathrm{E}+17$ & 4.63E-09 & $9.3 \mathrm{E}-10$ & 1 \\
\hline$X M M-\mathrm{OM}$ UVW2 & $1.41 \mathrm{E}+15$ & $1.94 \mathrm{E}-04$ & 1.7E-06 & 1 \\
\hline$X M M-O M$ UVW1 & $1.03 \mathrm{E}+15$ & $2.10 \mathrm{E}-04$ & 4.8E-06 & 1 \\
\hline$X M M-\mathrm{OM} \mathrm{U}$ & $8.71 \mathrm{E}+14$ & $4.28 \mathrm{E}-04$ & $3.0 \mathrm{E}-05$ & 1 \\
\hline HST ACS F435W (B) & $6.89 \mathrm{E}+14$ & 8.84E-04 & $1.6 \mathrm{E}-05$ & 1 \\
\hline HST ACS F814W (I) & $3.68 \mathrm{E}+14$ & $2.50 \mathrm{E}-03$ & 2.1E-05 & 1 \\
\hline Spitzer $24 \mu \mathrm{m}$ & $1.27 \mathrm{E}+13$ & $3.62 \mathrm{E}-02$ & 7.2E-03 & 1 \\
\hline $20 \mathrm{~cm}$ & $1.47 \mathrm{E}+09$ & $2.60 \mathrm{E}-03$ & $5.0 \mathrm{E}-05$ & 12 \\
\hline
\end{tabular}

Notes. The reference codes in Column 5 are as follows-1: new measurements in this article; 12: Mazzarella et al. (1988).

${ }^{a}$ The 70 and $160 \mu \mathrm{m}$ flux densities for the SW and NE galaxies were estimated using the 8 and $24 \mu \mathrm{m}$ measurements as described in the text. 
Table 14

Regions Covered in Published IRS Measurements

\begin{tabular}{|c|c|c|c|}
\hline Feature & $\begin{array}{c}\text { Flux } \\
\left(\mathrm{W} \mathrm{m}^{-2}\right) \\
(2)\end{array}$ & $\begin{array}{c}\text { EQW } \\
(\mu \mathrm{m}) \\
(3)\end{array}$ & $\begin{array}{c}\text { References } \\
\text { (4) }\end{array}$ \\
\hline \multicolumn{4}{|c|}{ Mrk 266 SW } \\
\hline [S IV] $10.51 \mu \mathrm{m}(\mathrm{SH})$ & $9.00( \pm 0.38) \times 10^{-21}$ & $\ldots$ & B09 \\
\hline $\mathrm{PAH} 10.6 \mu \mathrm{m}(\mathrm{SH})$ & $0.25( \pm 0.05) \times 10^{-20}$ & $\ldots$ & B09 \\
\hline $\mathrm{PAH} 10.7 \mu \mathrm{m}(\mathrm{SH})$ & $0.12( \pm 0.03) \times 10^{-20}$ & $\ldots$ & B09 \\
\hline PAH $11.0 \mu \mathrm{m}(\mathrm{SH})$ & $1.09( \pm 0.05) \times 10^{-20}$ & $\ldots$ & B09 \\
\hline PAH $11.3 \mu \mathrm{m}(\mathrm{SH})$ & $14.90( \pm 0.74) \times 10^{-20}$ & $\ldots$ & B09 \\
\hline PAH $12.0 \mu \mathrm{m}(\mathrm{SH})$ & $0.73( \pm 0.10) \times 10^{-20}$ & $\ldots$ & B09 \\
\hline $\mathrm{H}_{2}$ (S2) $12.28 \mu \mathrm{m}(\mathrm{SH})$ & $3.72( \pm 0.32) \times 10^{-21}$ & $\ldots$ & B09 \\
\hline $\mathrm{HI}(7-6) 12.37 \mu \mathrm{m}(\mathrm{SH})$ & $0.69( \pm 0.05) \times 10^{-21}$ & $\ldots$ & B09 \\
\hline $\mathrm{PAH} 12.74 \mu \mathrm{m}(\mathrm{SH})$ & $7.13( \pm 0.36) \times 10^{-20}$ & $\ldots$ & B09 \\
\hline [Ne II] $12.81 \mu \mathrm{m}(\mathrm{SH})$ & $57.04( \pm 1.64) \times 10^{-21}$ & $\begin{array}{l}\cdots \\
\cdots\end{array}$ & B09 \\
\hline $\mathrm{PAH} 13.5 \mu \mathrm{m}(\mathrm{SH})$ & $0.13( \pm 0.06) \times 10^{-21}$ & $\ldots$ & B09 \\
\hline $\mathrm{PAH} 14.2 \mu \mathrm{m}(\mathrm{SH})$ & $0.57( \pm 0.06) \times 10^{-20}$ & $\cdots$ & B09 \\
\hline$[\mathrm{Ne} \mathrm{v}] 14.32 \mu \mathrm{m}(\mathrm{SH})$ & $7.96( \pm 0.17) \times 10^{-21}$ & $\ldots$ & B09 \\
\hline$[\mathrm{Cl}$ II $] 14.38 \mu \mathrm{m}(\mathrm{SH})$ & $0.46( \pm 0.07) \times 10^{-21}$ & $\ldots$ & B09 \\
\hline [Ne III] $15.55 \mu \mathrm{m}(\mathrm{SH})$ & $27.95( \pm 0.76) \times 10^{-21}$ & $\ldots$ & B09 \\
\hline PAH $16.45 \mu \mathrm{m}(\mathrm{SH})$ & $0.84( \pm 0.08) \times 10^{-20}$ & $\ldots$ & B09 \\
\hline $\mathrm{PAH} 17.0 \mu \mathrm{m}(\mathrm{SH})$ & $1.07( \pm 0.14) \times 10^{-20}$ & $\cdots$ & B09 \\
\hline $\mathrm{H}_{2}(\mathrm{~S} 1) 17.03 \mu \mathrm{m}(\mathrm{SH})$ & $8.40( \pm 0.97) \times 10^{-21}$ & $\ldots$ & B09 \\
\hline [P III] $17.89 \mu \mathrm{m}(\mathrm{SH})$ & $0.67( \pm 0.15) \times 10^{-21}$ & $\ldots$ & B09 \\
\hline$[\mathrm{Fe} \mathrm{II}] 17.95 \mu \mathrm{m}(\mathrm{SH})$ & $0.48( \pm 0.14) \times 10^{-21}$ & $\cdots$ & B09 \\
\hline [S III] $18.71 \mu \mathrm{m}(\mathrm{SH})$ & $24.33( \pm 0.69) \times 10^{-21}$ & $\ldots$ & B09 \\
\hline \multicolumn{4}{|c|}{ Mrk 266 SW + Source between Nucleus } \\
\hline$[\mathrm{Ne}$ III $] 15.55 \mu \mathrm{m}(\mathrm{LL})^{\mathrm{a}}$ & & $\ldots$ & B06 \\
\hline PAH $17.0 \mu \mathrm{m}(\mathrm{LL})$ & $0.51 \times 10^{-19}$ & 0.403 & B06 \\
\hline [S III] $18.71 \mu \mathrm{m}(\mathrm{LL})^{\mathrm{a}}$ & $\ldots$ & $\ldots$ & B06 \\
\hline [Fe III] $22.93 \mu \mathrm{m}(\mathrm{LH})$ & $2.15( \pm 0.29) \times 10^{-21}$ & $\cdots$ & B09 \\
\hline$[\mathrm{Ne} \mathrm{v}] 24.31 \mu \mathrm{m}(\mathrm{LH})^{\mathrm{b}}$ & $11.12( \pm 0.59) \times 10^{-21}$ & $\ldots$ & B09 \\
\hline [O IV] $25.89 \mu \mathrm{m}(\mathrm{LH})$ & $52.94( \pm 5.66) \times 10^{-21}$ & $\ldots$ & B09 \\
\hline [O Iv] $25.89 \mu \mathrm{m}(\mathrm{LL})^{\mathrm{a}}$ & $\ldots$ & $\ldots$ & B06 \\
\hline$[\mathrm{Fe}$ II] $25.98 \mu \mathrm{m}(\mathrm{LH})$ & $4.35( \pm 0.70) \times 10^{-21}$ & $\ldots$ & B09 \\
\hline $\mathrm{S}_{2}(\mathrm{~S} 0) 28.22 \mu \mathrm{m}(\mathrm{LH})$ & $<1.60 \times 10^{-21}$ & $\ldots$ & B09 \\
\hline [S III] $33.48 \mu \mathrm{m}(\mathrm{LH})$ & $50.92( \pm 2.91) \times 10^{-21}$ & $\ldots$ & B09 \\
\hline$\left[\mathrm{S}_{\text {III }}\right] 33.48 \mu \mathrm{m}(\mathrm{LL})^{\mathrm{a}}$ & $\ldots$ & $\ldots$ & B06 \\
\hline [Si II] $34.81 \mu \mathrm{m}(\mathrm{LH})$ & $87.02( \pm 4.44) \times 10^{-21}$ & $\ldots$ & B09 \\
\hline \multicolumn{4}{|c|}{ Mrk 266 NE } \\
\hline PAH $6.2 \mu \mathrm{m}(\mathrm{SL})$ & $0.59 \times 10^{-19}$ & 0.619 & B06 \\
\hline $\mathrm{PAH} 7.7 \mu \mathrm{m}(\mathrm{SL})$ & $0.92 \times 10^{-19}$ & 0.467 & B06 \\
\hline PAH $8.6 \mu \mathrm{m}(\mathrm{SL})$ & $\ldots$ & 0.126 & B06 \\
\hline $\mathrm{H}_{2} \mathrm{~S}(3) 9.665 \mu \mathrm{m}(\mathrm{SL})^{\mathrm{a}}$ & $\ldots$ & $\ldots$ & B06 \\
\hline$\left[\mathrm{S}_{\text {IV }}\right] 10.51 \mu \mathrm{m}(\mathrm{SH})^{\mathrm{a}}$ & $\ldots$ & $\ldots$ & B06 \\
\hline $\mathrm{H}_{2} \mathrm{~S}(2) 12.279 \mu \mathrm{m}(\mathrm{SL})^{\mathrm{a}}$ & $\ldots$ & $\ldots$ & B06 \\
\hline$[\mathrm{Ne}$ II $] 12.81 \mu \mathrm{m}(\mathrm{SL})^{\mathrm{a}}$ & $\ldots$ & $\ldots$ & B06 \\
\hline PAH $11.3 \mu \mathrm{m}(\mathrm{SL})$ & $0.47 \times 10^{-19}$ & 0.422 & B06 \\
\hline PAH $14.2 \mu \mathrm{m}(\mathrm{SL})$ & $0.08 \times 10^{-19}$ & 0.065 & B06 \\
\hline
\end{tabular}

Notes. Column 1: spectral line identification followed in parentheses by the IRS module used: SL $=$ $5.2-14.5 \mu \mathrm{m}, 33^{\prime \prime} 7 \times 57^{\prime \prime}, R \sim 60-127 ; \mathrm{LL}=14.0-38.0 \mu \mathrm{m}, 10^{\prime \prime} 5 \times 168^{\prime \prime} 3, R \sim 57-126 ; \mathrm{SH}=$ 9.9-19.6 $\mu \mathrm{m}, 4$.'7 $\times 11^{\prime \prime} .3, R \sim 600 ; \mathrm{LH}=18.7-37.3 \mu \mathrm{m}, 11^{\prime \prime} .1 \times 22^{\prime \prime} .3, R \sim 600$. Column 2: flux in $\mathrm{W} \mathrm{cm}^{-2}$. Column 3: equivalent width in $\mu \mathrm{m}$. Column 4: Reference: B06: Brandl et al. (2006); B09: Bernard-Salas et al. (2009).

${ }^{a}$ These features are visible in the low-res spectrum published by Brandl et al. (2006), but fluxes were not published.

$\mathrm{b}$ This is consistent with an [Ne v] $24.32 \mu \mathrm{m}$ flux of $1.19( \pm 0.06) \times 10^{-20} \mathrm{~W} \mathrm{~cm}^{-2}$ measured from the same LH aperture by Dudik et al. (2007, Table 2).

AOR 3755264 also contained high-resolution observations (spectral resolution 600). Dudik et al. (2007, Figure 8) plotted the SH and LH apertures of these observations as an overlay on a $20 \mathrm{~cm}$ image, although they did not present the resulting spectra; their diagram clearly illustrates the aperture affects that complicate the interpretation of IRS spectra for this system. The SH slit contained only the radio source between the nuclei (missing both nuclei), while the LH slit contained $266 \mathrm{SW}$ and the central radio source but it missed Mrk 266 NE. Observations with the SH $(9.9-19.6 \mu \mathrm{m})$ module were also obtained on 2005 
January 10 in Spitzer program P03237 (PI: E. Sturm, AOR 10510592); these data cover the SW nucleus, but not the NE nucleus. The available high-resolution spectra were published in an atlas of Spitzer spectra for a sample of starburst galaxies (Bernard-Salas et al. 2009).

\section{A.3. Evidence That Fe Ko Originates in Mrk 266 SW}

Another clue to the origin of the $\mathrm{Fe} \mathrm{K} \alpha$ emission in Mrk 266 comes from analysis of the X-ray data in combination with optical spectroscopy. The ratio of X-ray (2-10 keV) continuum flux to (dereddened) [O III] 25007 emission-line flux has been shown to be anti-correlated with $N_{\mathrm{H}}$ and with the equivalent width of the $\mathrm{Fe} \mathrm{K} \alpha$ line, thus providing an indicator for Compton-thick AGNs (Bassani et al. 1999). Using the $F_{x}$ (hard) values measured from Chandra (Table 2) and the de-reddened [O III] $\lambda 5007$ line fluxes from Veilleux et al. (1995) $\left(7.8 \times 10^{-14}\right.$ and $8.5 \times 10^{-15} \mathrm{erg} \mathrm{s}^{-1}$ for the SW and NE nuclei), the $F_{x} / F_{\text {[O III] }}$ ratios are 0.28 and 16 for the $S W$ and NE nuclei, respectively. A ratio of $F_{x} / F_{\text {[O III] }} \lesssim 0.1$ indicates a highly absorbed source $\left(N_{\mathrm{H}} \gtrsim 10^{24} \mathrm{~cm}^{-2}\right)$, whereas $F_{x} / F_{\text {[O III] }} \gg 1$ is a signature of a relatively unobscured (Type 1) AGN (Bassani et al. 1999). This result adds compelling evidence that the $\mathrm{Fe} \mathrm{K} \alpha$ line detected in the XMM spectrum of Mrk 266 originates from the SW (Seyfert 2) nucleus. Although Mrk 266 SW is a strong candidate for containing a Compton-thick X-ray source, observations at higher energies and greater sensitivity are required for confirmation.

\section{A.4. Star Cluster Photometry (Section 3.6)}

Coordinates and photometric properties of SCs are listed in Table 15.

Table 15

Star Cluster Photometry

\begin{tabular}{|c|c|c|c|c|c|c|c|c|c|}
\hline SC\# & $\begin{array}{c}\delta \text { R.A. } \\
\text { (ss.ss) } \\
(2)\end{array}$ & $\begin{array}{c}\delta \text { Decl. } \\
\text { (mm ss.s) } \\
(3)\end{array}$ & $\begin{array}{c}B \\
(\mathrm{mag}) \\
(4)\end{array}$ & $\begin{array}{c}\sigma \\
(\mathrm{mag}) \\
(5)\end{array}$ & $\begin{array}{c}M_{\mathrm{B}} \\
(\mathrm{mag}) \\
(6)\end{array}$ & $\begin{array}{c}I \\
(\mathrm{mag}) \\
(7)\end{array}$ & $\begin{array}{c}\sigma \\
(\mathrm{mag}) \\
(8)\end{array}$ & $\begin{array}{c}B-I \\
(\mathrm{mag}) \\
(9)\end{array}$ & $\begin{array}{c}\sigma \\
(\mathrm{mag}) \\
(10)\end{array}$ \\
\hline 001 & 17.19 & 1632.4 & 21.45 & 0.03 & -14.16 & 21.11 & 0.05 & 0.31 & 0.06 \\
\hline 002 & 17.35 & 1631.9 & 21.52 & 0.04 & -14.09 & 19.48 & 0.04 & 2.01 & 0.06 \\
\hline 003 & 17.14 & 1633.9 & 22.50 & 0.04 & -13.11 & 21.25 & 0.08 & 1.22 & 0.09 \\
\hline 004 & 17.35 & 1630.9 & 22.91 & 0.13 & -12.70 & 21.20 & 0.08 & 1.68 & 0.15 \\
\hline 005 & 18.40 & 1643.8 & 23.07 & 0.01 & -12.54 & 22.47 & 0.03 & 0.57 & 0.03 \\
\hline 006 & 17.39 & 1630.9 & 23.08 & 0.07 & -12.53 & 21.21 & 0.05 & 1.84 & 0.09 \\
\hline 007 & 17.16 & 1636.8 & 23.14 & 0.05 & -12.47 & 22.34 & 0.09 & 0.77 & 0.10 \\
\hline 008 & 17.17 & 1636.5 & 23.24 & 0.06 & -12.37 & 22.58 & 0.08 & 0.63 & 0.10 \\
\hline 009 & 17.72 & 1634.1 & 23.53 & 0.02 & -12.08 & 22.83 & 0.03 & 0.67 & 0.04 \\
\hline 010 & 17.02 & 1636.6 & 23.54 & 0.09 & -12.07 & 22.49 & 0.13 & 1.02 & 0.16 \\
\hline 011 & 17.08 & 1634.7 & 23.63 & 0.04 & -11.98 & 20.91 & 0.02 & 2.69 & 0.04 \\
\hline 012 & 16.96 & 1632.0 & 23.84 & 0.09 & -11.77 & 23.17 & 0.20 & 0.64 & 0.22 \\
\hline 013 & 16.15 & 1639.9 & 23.97 & 0.01 & -11.64 & 22.65 & 0.01 & 1.29 & 0.01 \\
\hline 014 & 16.79 & 1644.4 & 24.00 & 0.03 & -11.61 & 23.24 & 0.04 & 0.73 & 0.05 \\
\hline 015 & 16.83 & 1639.2 & 24.09 & 0.10 & -11.52 & 23.87 & 0.16 & 0.19 & 0.19 \\
\hline 016 & 17.68 & 1638.4 & 24.20 & 0.04 & -11.41 & 23.37 & 0.16 & 0.80 & 0.16 \\
\hline 017 & 17.45 & 1628.8 & 24.23 & 0.13 & -11.38 & 22.76 & 0.14 & 1.44 & 0.19 \\
\hline 018 & 18.64 & 1644.5 & 24.28 & 0.06 & -11.33 & 23.80 & 0.07 & 0.45 & 0.09 \\
\hline 019 & 17.06 & 1631.9 & 24.30 & 0.15 & -11.31 & 23.23 & 0.22 & 1.04 & 0.27 \\
\hline 020 & 16.80 & 1636.2 & 24.40 & 0.07 & -11.21 & 23.45 & 0.13 & 0.92 & 0.15 \\
\hline 021 & 16.91 & 1645.6 & 24.54 & 0.03 & -11.07 & 23.82 & 0.04 & 0.69 & 0.05 \\
\hline 022 & 16.71 & 1640.7 & 24.60 & 0.03 & -11.01 & 23.74 & 0.04 & 0.83 & 0.05 \\
\hline 023 & 18.16 & 1642.3 & 24.71 & 0.07 & -10.90 & 25.32 & 0.81 & -0.64 & 0.81 \\
\hline 024 & 17.22 & 1635.2 & 24.72 & 0.08 & -10.89 & 22.91 & 0.12 & 1.78 & 0.14 \\
\hline 025 & 16.62 & 1645.5 & 24.73 & 0.04 & -10.88 & 23.74 & 0.04 & 0.96 & 0.06 \\
\hline 026 & 17.27 & 1627.4 & 24.74 & 0.11 & -10.87 & 23.43 & 0.16 & 1.28 & 0.19 \\
\hline 027 & 17.29 & 1628.4 & 24.76 & 0.26 & -10.85 & 23.47 & 0.32 & 1.26 & 0.41 \\
\hline 028 & 17.57 & 1642.1 & 24.83 & 0.19 & -10.78 & 23.83 & 0.19 & 0.97 & 0.27 \\
\hline 029 & 17.19 & 1630.1 & 24.85 & 0.12 & -10.76 & 25.81 & 1.05 & -0.99 & 1.06 \\
\hline 030 & 16.32 & 1641.0 & 24.86 & 0.02 & -10.75 & 22.23 & 0.01 & 2.60 & 0.02 \\
\hline 031 & 16.77 & 1645.2 & 24.91 & 0.04 & -10.70 & 24.22 & 0.06 & 0.66 & 0.07 \\
\hline 032 & 16.97 & 1644.4 & 24.92 & 0.07 & -10.69 & 23.74 & 0.05 & 1.15 & 0.09 \\
\hline 033 & 17.09 & 1637.9 & 24.92 & 0.15 & -10.69 & 23.32 & 0.16 & 1.57 & 0.22 \\
\hline 034 & 16.53 & 1631.3 & 24.94 & 0.03 & -10.67 & 23.81 & 0.04 & 1.10 & 0.05 \\
\hline 035 & 17.23 & 1640.6 & 24.95 & 0.08 & -10.66 & 24.73 & 0.22 & 0.19 & 0.23 \\
\hline 036 & 18.48 & 1645.2 & 24.97 & 0.05 & -10.64 & 24.43 & 0.07 & 0.51 & 0.09 \\
\hline 037 & 18.45 & 1636.6 & 25.00 & 0.08 & -10.61 & 24.33 & 0.21 & 0.64 & 0.22 \\
\hline 038 & 18.69 & 1644.3 & 25.00 & 0.07 & -10.61 & 24.62 & 0.13 & 0.35 & 0.15 \\
\hline 039 & 17.94 & 1631.3 & 25.05 & 0.06 & -10.56 & 24.07 & 0.07 & 0.95 & 0.09 \\
\hline 040 & 18.38 & 1645.9 & 25.12 & 0.04 & -10.49 & 24.27 & 0.08 & 0.82 & 0.09 \\
\hline 041 & 16.76 & 1645.6 & 25.13 & 0.07 & -10.48 & 24.64 & 0.11 & 0.46 & 0.13 \\
\hline 042 & 18.47 & 1704.5 & 25.14 & 0.03 & -10.47 & 24.17 & 0.03 & 0.94 & 0.04 \\
\hline 043 & 18.73 & 1644.9 & 25.16 & 0.11 & -10.45 & 24.10 & 0.11 & 1.03 & 0.16 \\
\hline 044 & 16.97 & 1643.0 & 25.19 & 0.07 & -10.42 & 24.11 & 0.08 & 1.05 & 0.11 \\
\hline
\end{tabular}


Table 15

(Continued)

\begin{tabular}{|c|c|c|c|c|c|c|c|c|c|}
\hline SC\# & $\begin{array}{c}\delta \text { R.A. } \\
\text { (ss.ss) } \\
(2)\end{array}$ & $\begin{array}{c}\delta \text { Decl. } \\
\text { (mm ss.s) } \\
(3)\end{array}$ & $\begin{array}{c}B \\
(\mathrm{mag}) \\
(4)\end{array}$ & $\begin{array}{c}\sigma \\
(\mathrm{mag}) \\
(5)\end{array}$ & $\begin{array}{c}M_{\mathrm{B}} \\
(\mathrm{mag}) \\
(6)\end{array}$ & $\begin{array}{c}I \\
(\mathrm{mag}) \\
(7)\end{array}$ & $\begin{array}{c}\sigma \\
(\mathrm{mag}) \\
(8)\end{array}$ & $\begin{array}{c}B-I \\
(\mathrm{mag}) \\
(9)\end{array}$ & $\begin{array}{c}\sigma \\
(\mathrm{mag}) \\
(10)\end{array}$ \\
\hline 045 & 16.70 & 1637.2 & 25.20 & 0.13 & -10.41 & 24.57 & 0.25 & 0.60 & 0.28 \\
\hline 046 & 16.92 & 1642.2 & 25.24 & 0.06 & -10.37 & 24.46 & 0.07 & 0.75 & 0.09 \\
\hline 047 & 17.21 & 1624.6 & 25.28 & 0.04 & -10.33 & 24.63 & 0.06 & 0.62 & 0.07 \\
\hline 048 & 16.77 & 1638.7 & 25.32 & 0.14 & -10.29 & 24.72 & 0.31 & 0.57 & 0.34 \\
\hline 049 & 17.16 & 1628.1 & 25.34 & 0.28 & -10.27 & 24.01 & 0.34 & 1.30 & 0.44 \\
\hline 050 & 17.22 & 1626.0 & 25.35 & 0.07 & -10.26 & 24.30 & 0.08 & 1.02 & 0.11 \\
\hline 051 & 16.42 & 1630.9 & 25.36 & 0.05 & -10.25 & 23.23 & 0.03 & 2.10 & 0.06 \\
\hline 052 & 18.32 & 1643.7 & 25.36 & 0.09 & -10.25 & 24.48 & 0.22 & 0.85 & 0.24 \\
\hline 053 & 17.20 & 1643.2 & 25.36 & 0.06 & -10.25 & 24.24 & 0.10 & 1.09 & 0.12 \\
\hline 054 & 17.00 & 1634.6 & 25.38 & 0.22 & -10.23 & 24.57 & 0.58 & 0.78 & 0.62 \\
\hline 055 & 18.57 & 1651.2 & 25.39 & 0.04 & -10.22 & 24.32 & 0.03 & 1.04 & 0.05 \\
\hline 056 & 18.56 & 1644.9 & 25.42 & 0.07 & -10.19 & 24.91 & 0.10 & 0.48 & 0.12 \\
\hline 057 & 16.77 & 1632.1 & 25.48 & 0.27 & -10.13 & 24.40 & 0.33 & 1.05 & 0.43 \\
\hline 058 & 18.66 & 1645.6 & 25.48 & 0.13 & -10.13 & 24.41 & 0.11 & 1.04 & 0.17 \\
\hline 059 & 17.13 & 1644.7 & 25.50 & 0.06 & -10.11 & 24.80 & 0.10 & 0.67 & 0.12 \\
\hline 060 & 15.85 & 1642.5 & 25.50 & 0.04 & -10.11 & 24.53 & 0.04 & 0.94 & 0.06 \\
\hline 061 & 18.77 & 1642.3 & 25.51 & 0.08 & -10.10 & 24.97 & 0.12 & 0.51 & 0.14 \\
\hline 062 & 16.20 & 1633.6 & 25.53 & 0.04 & -10.08 & 24.37 & 0.03 & 1.13 & 0.05 \\
\hline 063 & 18.41 & 1637.7 & 25.53 & 0.09 & -10.08 & 24.57 & 0.20 & 0.93 & 0.22 \\
\hline 064 & 16.72 & 1635.3 & 25.53 & 0.18 & -10.08 & 23.99 & 0.19 & 1.51 & 0.26 \\
\hline 065 & 18.36 & 1645.4 & 25.54 & 0.07 & -10.07 & 25.06 & 0.16 & 0.45 & 0.17 \\
\hline 066 & 17.00 & 1643.0 & 25.59 & 0.10 & -10.02 & 24.76 & 0.16 & 0.80 & 0.19 \\
\hline 067 & 17.63 & 1631.7 & 25.60 & 0.16 & -10.01 & 24.06 & 0.19 & 1.51 & 0.25 \\
\hline 068 & 19.08 & 1613.2 & 25.62 & 0.04 & -9.99 & 24.03 & 0.02 & 1.56 & 0.04 \\
\hline 069 & 16.63 & 1646.2 & 25.64 & 0.11 & -9.97 & 24.75 & 0.13 & 0.86 & 0.17 \\
\hline 070 & 17.33 & 1640.6 & 25.65 & 0.18 & -9.96 & 24.68 & 0.17 & 0.94 & 0.25 \\
\hline 071 & 18.73 & 1643.8 & 25.65 & 0.22 & -9.96 & 25.84 & 0.51 & -0.22 & 0.56 \\
\hline 072 & 16.64 & 1638.9 & 25.66 & 0.09 & -9.95 & 24.82 & 0.14 & 0.81 & 0.17 \\
\hline 073 & 16.09 & 1631.2 & 25.68 & 0.04 & -9.93 & 24.51 & 0.04 & 1.14 & 0.06 \\
\hline 074 & 17.94 & 1637.0 & 25.70 & 0.10 & -9.91 & 24.86 & 0.30 & 0.81 & 0.32 \\
\hline 075 & 18.06 & 1635.4 & 25.70 & 0.12 & -9.91 & 25.18 & 0.29 & 0.49 & 0.31 \\
\hline 076 & 15.58 & 1641.0 & 25.72 & 0.05 & -9.89 & 24.68 & 0.04 & 1.01 & 0.06 \\
\hline 077 & 20.51 & 1618.3 & 25.77 & 0.05 & -9.84 & 24.57 & 0.04 & 1.17 & 0.06 \\
\hline 078 & 17.70 & 1634.7 & 25.77 & 0.15 & -9.84 & 25.10 & 0.33 & 0.64 & 0.36 \\
\hline 079 & 18.29 & 1643.1 & 25.80 & 0.12 & -9.81 & 24.49 & 0.22 & 1.28 & 0.25 \\
\hline 080 & 18.74 & 1643.6 & 25.82 & 0.20 & -9.79 & 26.29 & 0.62 & -0.50 & 0.65 \\
\hline 081 & 18.50 & 1648.8 & 25.92 & 0.08 & -9.69 & 25.25 & 0.08 & 0.64 & 0.11 \\
\hline 082 & 16.72 & 1645.3 & 25.95 & 0.22 & -9.66 & 25.45 & 0.27 & 0.47 & 0.35 \\
\hline 083 & 16.75 & 1637.9 & 26.02 & 0.25 & -9.59 & 24.63 & 0.19 & 1.36 & 0.31 \\
\hline 084 & 16.50 & 1646.1 & 26.06 & 0.07 & -9.55 & 24.71 & 0.06 & 1.32 & 0.09 \\
\hline 085 & 17.31 & 1639.9 & 26.08 & 0.20 & -9.53 & 25.29 & 0.19 & 0.76 & 0.28 \\
\hline 086 & 16.56 & 1645.3 & 26.08 & 0.12 & -9.53 & 25.45 & 0.20 & 0.60 & 0.23 \\
\hline 087 & 16.86 & 1645.9 & 26.12 & 0.13 & -9.49 & 25.54 & 0.23 & 0.55 & 0.26 \\
\hline 088 & 18.54 & 1645.3 & 26.13 & 0.14 & -9.48 & 25.81 & 0.28 & 0.29 & 0.31 \\
\hline 089 & 16.09 & 1646.2 & 26.17 & 0.09 & -9.44 & 24.96 & 0.07 & 1.18 & 0.11 \\
\hline 090 & 18.01 & 1628.7 & 26.19 & 0.11 & -9.42 & 24.78 & 0.09 & 1.38 & 0.14 \\
\hline 091 & 16.85 & 1638.1 & 26.19 & 0.40 & -9.42 & 25.87 & 0.63 & 0.29 & 0.75 \\
\hline 092 & 16.44 & 1648.2 & 26.20 & 0.08 & -9.41 & 25.42 & 0.11 & 0.75 & 0.14 \\
\hline 093 & 19.64 & 1613.9 & 26.23 & 0.07 & -9.38 & 25.11 & 0.05 & 1.09 & 0.09 \\
\hline 094 & 17.86 & 1632.2 & 26.24 & 0.13 & -9.37 & 24.23 & 0.08 & 1.98 & 0.15 \\
\hline 095 & 16.14 & 1642.6 & 26.27 & 0.09 & -9.34 & 25.22 & 0.09 & 1.02 & 0.13 \\
\hline 096 & 17.20 & 1626.4 & 26.29 & 0.16 & -9.32 & 26.50 & 0.79 & -0.24 & 0.81 \\
\hline 097 & 19.40 & 1636.0 & 26.30 & 0.09 & -9.31 & 25.37 & 0.09 & 0.90 & 0.13 \\
\hline 098 & 16.90 & 1627.0 & 26.30 & 0.18 & -9.31 & 24.45 & 0.12 & 1.82 & 0.22 \\
\hline 099 & 16.73 & 1635.7 & 26.31 & 0.41 & -9.30 & 24.08 & 0.21 & 2.20 & 0.46 \\
\hline 100 & 18.68 & 1650.1 & 26.43 & 0.09 & -9.18 & 25.79 & 0.12 & 0.61 & 0.15 \\
\hline 101 & 17.50 & 1616.2 & 26.46 & 0.09 & -9.15 & 25.47 & 0.08 & 0.96 & 0.12 \\
\hline 102 & 16.45 & 1628.4 & 26.50 & 0.11 & -9.11 & 26.66 & 0.39 & -0.19 & 0.41 \\
\hline 103 & 15.87 & 1639.3 & 26.51 & 0.09 & -9.10 & 25.41 & 0.09 & 1.07 & 0.13 \\
\hline 104 & 14.99 & 1628.8 & 26.53 & 0.09 & -9.08 & 25.14 & 0.07 & 1.36 & 0.11 \\
\hline 105 & 18.08 & 1617.9 & 26.55 & 0.10 & -9.06 & 25.66 & 0.10 & 0.86 & 0.14 \\
\hline 106 & 20.16 & 1624.9 & 26.56 & 0.10 & -9.05 & 25.16 & 0.06 & 1.37 & 0.12 \\
\hline 107 & 18.69 & 1645.9 & 26.56 & 0.36 & -9.05 & 25.34 & 0.27 & 1.19 & 0.45 \\
\hline 108 & 16.44 & 1635.0 & 26.58 & 0.13 & -9.03 & 25.16 & 0.13 & 1.39 & 0.18 \\
\hline 109 & 17.49 & 1611.3 & 26.63 & 0.09 & -8.98 & 25.69 & 0.10 & 0.91 & 0.13 \\
\hline
\end{tabular}




\begin{tabular}{|c|c|c|c|c|c|c|c|c|c|}
\hline \multicolumn{10}{|c|}{$\begin{array}{c}\text { Table } 15 \\
\text { (Continued) }\end{array}$} \\
\hline $\begin{array}{l}\text { SC\# } \\
\text { (1) }\end{array}$ & $\begin{array}{c}\delta \text { R.A. } \\
\text { (ss.ss) } \\
(2)\end{array}$ & $\begin{array}{c}\delta \text { Decl. } \\
\text { (mm ss.s) } \\
(3)\end{array}$ & $\begin{array}{c}B \\
(\mathrm{mag}) \\
(4)\end{array}$ & $\begin{array}{c}\sigma \\
(\mathrm{mag}) \\
(5)\end{array}$ & $\begin{array}{c}M_{\mathrm{B}} \\
(\mathrm{mag}) \\
(6)\end{array}$ & $\begin{array}{c}I \\
(\mathrm{mag}) \\
(7)\end{array}$ & $\begin{array}{c}\sigma \\
(\mathrm{mag}) \\
(8)\end{array}$ & $\begin{array}{c}B-I \\
(\mathrm{mag}) \\
(9)\end{array}$ & $\begin{array}{c}\sigma \\
(\mathrm{mag}) \\
(10)\end{array}$ \\
\hline 110 & 15.71 & 1624.7 & 26.63 & 0.10 & -8.98 & 25.49 & 0.09 & 1.11 & 0.13 \\
\hline 111 & 15.77 & 1644.5 & 26.76 & 0.12 & -8.85 & 24.62 & 0.05 & 2.11 & 0.13 \\
\hline 112 & 17.22 & 1644.0 & 26.77 & 0.21 & -8.84 & 25.77 & 0.41 & 0.97 & 0.46 \\
\hline 113 & 16.42 & 1646.4 & 26.85 & 0.16 & -8.76 & 25.87 & 0.19 & 0.95 & 0.25 \\
\hline 114 & 16.04 & 1621.8 & 26.89 & 0.12 & -8.72 & 26.14 & 0.16 & 0.72 & 0.20 \\
\hline 115 & 16.78 & 1657.1 & 27.07 & 0.18 & -8.54 & 25.73 & 0.12 & 1.31 & 0.22 \\
\hline 116 & 18.37 & 1640.5 & 27.12 & 0.88 & -8.49 & 24.25 & 0.28 & 2.84 & 0.92 \\
\hline 117 & 15.93 & 1650.7 & 27.13 & 0.17 & -8.48 & 25.98 & 0.13 & 1.12 & 0.21 \\
\hline 118 & 15.67 & 1648.2 & 27.16 & 0.13 & -8.45 & 25.59 & 0.09 & 1.54 & 0.16 \\
\hline 119 & 17.56 & 1656.9 & 27.23 & 0.22 & -8.38 & 25.73 & 0.13 & 1.47 & 0.26 \\
\hline
\end{tabular}

Notes. Column 1: star cluster (candidate) number. Columns 2 and 3: J2000 $\delta$ R.A. and $\delta$ decl. of the object. To save space, offsets in seconds of time in R.A. and offsets in arcminutes and arcseconds in decl. are listed. Full coordinates are obtained by adding these values to R.A. $=13^{\mathrm{h}} 38^{\mathrm{m}}$ and decl. $=48^{\mathrm{d}}$ (e.g., $13^{\mathrm{h}} 38^{\mathrm{m}} 17.19 ; 48^{\mathrm{d}} 16^{\mathrm{m}} 32^{\mathrm{s}} .4$ ). The $1 \sigma$ uncertainty in the coordinates is $\pm 0{ }^{\prime \prime} 3$. The objects can be identified as "Mrk 266 SC\#\#\#" or as "GOALS Jhhmmssss+ddmmsss." For example: Mrk 266 SC001 = GOALS J13381719+4816324. Column 4: apparent mag in the HST ACS F435W (B) filter. Column 5: $1 \sigma$ uncertainty in the B mag. Column 6: absolute B mag. Column 7: apparent mag in the HST ACS F814W (I) filter. Column 8: $1 \sigma$ uncertainty in the $I$ mag. Column 9: $B-I$ color mag. Column 10: $1 \sigma$ uncertainty in the $B-I$ color mag. Absolute $B$ mag and $B-I$ colors have been corrected for foreground Galactic extinction using values from Schlegel et al. (1998) as provided by NED: $A_{B}=0.056 \mathrm{mag} ; A_{I}=0.025 \mathrm{mag}$. These data have $n o t$ been corrected for extinction within Mrk 266 (see Section 3.6.2).

\section{REFERENCES}

Alton, P. B., Davies, J. I., \& Bianchi, S. 1999, A\&A, 343, 51

Appleton, P. N., Xu, K. C., Reach, W., et al. 2006, ApJ, 639, L51

Armus, L., Charmandaris, V., Bernard-Salas, J., et al. 2007, ApJ, 656, 148

Armus, L., Heckman, T. M., \& Miley, G. K. 1990, ApJ, 364, 471

Armus, L., Mazzarella, J. M., Evans, A. S., et al. 2009, PASP, 121, 559

Arnouts, S., Walcher, C. J., Le Fèvre, O., et al. 2007, A\&A, 476, 137

Awaki, H., Koyama, K., Inoue, H., \& Halpern, J. P. 1991, PASJ, 43, 195

Ballo, L., Braito, V., Della Ceca, R., et al. 2004, ApJ, 600, 634

Barnes, J. E., \& Hernquist, L. 1996, ApJ, 471, 115

Bassani, L., Dadina, M., Maiolino, R., et al. 1999, ApJS, 121, 473

Becker, R. H., White, R. L., \& Edwards, A. L. 1991, ApJS, 75, 1

Bernard-Salas, J., Spoon, H. W. W., Charmandaris, V., et al. 2009, ApJS, 184, 230

Bertin, E., \& Arnouts, S. 1996, A\&AS, 117, 393

Bianchi, S., Chiaberge, M., Piconcelli, E., Guainazzi, M., \& Matt, G. 2008, MNRAS, 386, 105

Bird, J., Martini, P., \& Kaiser, C. 2008, ApJ, 676, 147

Boroson, T. A., \& Lauer, T. R. 2009, Nature, 458, 53

Brandl, B. R., Bernard-Salas, J., Spoon, H. W. W., et al. 2006, ApJ, 653, 1129

Brassington, N. J., Ponman, T. J., \& Read, A. M. 2007, MNRAS, 377, 1439

Bruzual, G., \& Charlot, S. 2003, MNRAS, 344, 1000

Bryant, P. M., \& Scoville, N. Z. 1999, AJ, 117, 2632

Burke-Spolaor, S. 2011, MNRAS, 410, 2113

Calzetti, D., Kinney, A. L., \& Storchi-Bergmann, T. 1994, ApJ, 429, 582

Caputi, K. I., Lagache, G., Yan, L., et al. 2007, ApJ, 660, 97

Cecil, G. 1988, ApJ, 329, 38

Civano, F., Elvis, M., Lanzuisi, G., et al. 2010, ApJ, 717, 209

Cluver, M. E., Appleton, P. N., Boulanger, F., et al. 2010, ApJ, 710, 248

Cole, S., Norberg, P., Baugh, C. M., et al. 2001, MNRAS, 326, 255

Colpi, M., \& Dotti, M. 2009, arXiv:0906.4339

Comerford, J. M., Griffith, R. L., Gerke, B. F., et al. 2009, ApJ, 702, L82

Condon, J. J., Helou, G., Sanders, D. B., \& Soifer, B. T. 1993, AJ, 105, 1730

Cooper, J. L., Bicknell, G. V., Sutherland, R. S., \& Bland-Hawthorn, J. 2008, ApJ, 674, 157

Cox, T. J., Jonsson, P., Primack, J. R., \& Somerville, R. S. 2006, MNRAS, 373, 1013

Dasyra, K. M., Ho, L. C., Armus, L., et al. 2008, ApJ, 674, L9

Davies, R., Ward, M., \& Sugai, H. 2000, ApJ, 535, 735

de Propris, R., Eisenhardt, P. R., Stanford, S. A., \& Dickinson, M. 1998, ApJ, 503, L45

de Vaucouleurs, G., de Vaucouleurs, A., Corwin, H. G., Jr. (ed.), et al. 1991, Third Reference Catalogue of Bright Galaxies, Vol. I: Explanations and References, Vol. II: Data for Galaxies Between $0 \mathrm{hr}$ and $12 \mathrm{hr}$, Volume III.
Data for Galaxies Between $12 \mathrm{hr}$ and $24 \mathrm{hr}$, XII (Berlin: Springer), 2091 (RC3)

Douglas, J. N., Bash, F. N., Bozyan, F. A., Torrence, G. W., \& Wolfe, C. 1996, AJ, 111,1945

Downes, D., \& Solomon, P. M. 1998, ApJ, 507, 615

Draine, B. T., \& Li, A. 2001, ApJ, 551, 807

Dubinski, J., Mihos, J. C., \& Hernquist, L. 1996, ApJ, 462, 576

Dudik, R. P., Weingartner, J. C., Satyapal, S., et al. 2007, ApJ, 664, 71

Dunne, L., Eales, S., Edmunds, M., et al. 2000, MNRAS, 315, 115

Edelson, R. A. 1987, ApJ, 313, 651

Engel, H., Davies, R. I., Genzel, R., et al. 2010, A\&A, 524, A56

Evans, A. S., Mazzarella, J. M., Surace, J. A., et al. 2005, ApJS, 159, 197

Fazio, G. G., Hora, J. L., Allen, L. E., et al. 2004, ApJS, 154, 10

Ferrarese, L., \& Ford, H. 2005, Space Sci. Rev., 116, 523

Ford, H., Crane, P., Jacoby, G., et al. 1985, ApJ, 293, 132

Gaskell, C. M. 2010, Nature, 463, E1

Gavazzi, G., Pierini, D., \& Boselli, A. 1996, A\&A, 312, 397

Ghisellini, G., Haardt, F., \& Matt, G. 1994, MNRAS, 267, 743

Gieles, M., Larsen, S. S., Bastian, N., \& Stein, I. T. 2006, A\&A, 450, 129

Gonzalez-Martin, O., Masegosa, J., Mrquez, I., Guerrero, M. A., \& DultzinHacyan, D. 2006, A\&A, 460, 45

Graciá-Carpio, J., García-Burillo, S., \& Planesas, P. 2008, Ap\&SS, 313, 331

Grimes, J. P., Heckman, T., Strickland, D., \& Ptak, A. 2005, ApJ, 628, 187

Hales, S. E. G., Masson, C. R., Warner, P. J., \& Baldwin, J. E. 1990, MNRAS, 246, 256

Heckman, T. M., Armus, L., \& Miley, G. K. 1990, ApJS, 74, 833

Hildebrand, R. H. 1983, Q. J. R. Astron. Soc., 24, 267

Hinshaw, G., Weiland, J. L., Hill, R. S., et al. 2009, ApJS, 180, 225

Ho, L. C. 2008, ARA\&A, 46, 475

Hony, S., Van Kerckhoven, C., Peeters, E., et al. 2001, A\&A, 370, 1030

Hopkins, P. F., Hernquist, L., Cox, T. J., et al. 2006, ApJS, 163, 1

Houck, J. R., Roellig, T. L., van Cleve, J., et al. 2004, ApJS, 154, 18

Howell, J. H., Armus, L., Mazzarella, J. M., et al. 2010, ApJ, 715, 572

Huchra, J. P. 1977, ApJS, 35, 171

Hutchings, J. B., Neff, S. G., \& van Gorkom, J. H. 1988, AJ, 96, 1227

Imanishi, M., Nakanishi, K., Tamura, Y., Oi, N., \& Kohno, K. 2007, AJ, 134, 2366

Imanishi, M., Nakanishi, K., Tamura, Y., \& Peng, C.-H. 2009, AJ, 137, 3581

Inami, H., Armus, L., Surace, J. A., et al. 2010, AJ, 140, 63

Ishida, C. M. 2004, PhD thesis, IfA, Univ. Hawaii

Ishigaki, T., Yoshida, M., Aoki, K., et al. 2000, PASJ, 52, 185

Iwasawa, K., Crawford, C. S., Fabian, A. C., \& Wilman, R. J. 2005, MNRAS, 362, L20

Iwasawa, K., Sanders, D. B., Evans, A. S., et al. 2005, MNRAS, 357, 565

Iwasawa, K., Sanders, D. B., Evans, A. S., et al. 2009, ApJ, 695, L103

Iwasawa, K., Sanders, D. B., Teng, S. H., et al. 2011, A\&A, 529, A106 
Jarrett, T. H., Helou, G., Van Buren, D., Valjavec, E., \& Condon, J. J. 1999, AJ, 118,2132

Jiang, C. Y., Jing, Y. P., Faltenbacher, A., Lin, W. P., \& Li, C. 2008, ApJ, 675, 1095

Johansson, P. H., Burkert, A., \& Naab, T. 2009, ApJ, 707, L184

Karl, S. J., Naab, T., Johansson, P. H., et al. 2010, ApJ, 715, L88

Kewley, L. J., Groves, B., Kauffmann, G., \& Heckman, T. 2006, MNRAS, 372, 961

Khachikian, E., Ter-Kazarian, G., Sargsyan, L., Yerknapetyan, K., \& Terzian, Ye. 2006, MNRAS, 368, 461

Kim, D.-C., Sanders, D. B., Veilleux, S., Mazzarella, J. M., \& Soifer, B. T. 1995, ApJS, 98, 129

Kollatschny, W., \& Kowatsch, P. 1998, A\&A, 336, L21

Komossa, S., Burwitz, V., Hasinger, G., et al. 2003, ApJ, 582, L15

Koss, M., Mushotzky, R., Treister, E., et al. 2012, ApJ, 746, L22

Krist, J. 1993, in ASP Conf. Ser. 52, Astronomical Data Analysis Software and Systems II, ed. R. J. Hanisch, R. J. V. Brissenden, \& Jeannette Barnes (San Francisco, CA: ASP), 536

Krolik, J. H., Madau, P., \& Zycki, P. T. 1994, ApJ, 420, L57

Kuntz, K. D., Harrus, I., McGlynn, T., et al. 2008, PASP, 120, 740

Lacey, C. G., Baugh, C. M., Frenk, C. S., et al. 2008, MNRAS, 385, 1155

Larsen, S. S., \& Richtler, T. 1999, A\&A, 345, 59

Lauer, T. R., \& Boroson, T. A. 2009, ApJ, 703, 930

Laurent, O., Mirabel, I. F., Charmandaris, V., et al. 2000, A\&A, 359, 887

Le Floc'h, E., Papovich, C., Dole, H., et al. 2005, ApJ, 632, 169

Leipski, C., Falcke, H., Bennert, N., \& Huttemeister, S. 2006, A\&A, 455, 161

Lim, A. J. 2003, RevMexAA, 15, 131

Liu, X., Greene, J. E., Shen, Y., \& Strauss, M. A. 2010, ApJ, 715, L30

Magtesian, A. P., \& Khachikian, E. Ye. 1991, Sov. Astron. Lett., 17, 447

Marconi, A., \& Hunt, L. K. 2003, ApJ, 589, L21

Marleau, F. R., Fadda, D., Appleton, P. N., et al. 2007, ApJ, 663, 218

Marshall, J. A., Herter, T. L., Armus, L., et al. 2007, ApJ, 670, 129

Mason, K. O., Breeveld, A., Much, R., et al. 2001, A\&A, 365, L36

Mazzarella, J. M., \& Boroson, T. A. 1993, ApJS, 85, 27

Mazzarella, J. M., Bothun, G. D., \& Boroson, T. A. 1991a, AJ, 101, 2034

Mazzarella, J. M., Gaume, R. A., Aller, H. D., \& Hughes, P. A. 1988, ApJ, 333,168

Mazzarella, J. M., Soifer, B. T., Graham, J. R., et al. 1991b, AJ, 102, 1241

McCall, M. L. 2004, AJ, 128, 2144

Mihos, C. 1999, Ap\&SS, 266, 195

Mihos, J. C., \& Bothun, G. D. 1998, ApJ, 500, 619

Mihos, J. C., Dubinski, J., \& Hernquist, L. 1998, ApJ, 494, 183

Mihos, J. C., \& Hernquist, L. 1996, ApJ, 464, 641

Mirabel, I. F., Vigroux, L., Charmandaris, V., et al. 1998, A\&A, 333, L1

Modica, F., Vavilkin, T., Evans, A. S., et al. 2012, AJ, 143, 16

Mould, J. R., Huchra, J. P., Freedman, W. L., et al. 2000a, ApJ, 529, 786

Mould, J. R., Huchra, J. P., Freedman, W. L., et al. 2000b, ApJ, 545, 547

Murphy, T. W., Jr., Armus, L., Matthews, K., et al. 1996, AJ, 111, 1025

Nagar, N. M., Falcke, H., \& Wilson, A. S. 2005, A\&A, 435, 521

Nagar, N. M., Wilson, A. S., Falcke, H., et al. 2003, A\&A, 409, 115

Oesch, P. A., Carollo, C. M., Feldmann, R., et al. 2010, ApJ, 714, L47

Owen, F. N., O’Dea, C. P., Inoue, M., \& Eilek, J. A. 1985, ApJ, 294, L85

Peeters, E., Hony, S., Van Kerckhoven, C., et al. 2002, A\&A, 390, 1089

Peeters, E., Spoon, H. W. W., \& Tielens, A. G. G. M. 2004, ApJ, 613, 986

Peng, C. Y., Ho, L. C., Impey, C. D., \& Rix, H.-W. 2002, AJ, 124, 266

Petrosian, A. R., Saakian, K. A., \& Khachikian, E. E. 1978, Astrofizika, 14, 69

Petrosian, A. R., Saakian, K. A., \& Khachikian, E. E. 1979, Astrofizika, 15, 209
Petrosian, A. R., Saakian, K. A., \& Khachikian, E. E. 1980, Astrofizika, 16, 621 Ptak, A., Heckman, T., Levenson, N. A., Weaver, K., \& Strickland, D. 2003, ApJ, 592, 782

Radford, S. J. E., Downes, D., \& Solomon, P. M. 1991, ApJ, 368, L15

Ramos Almeida, C., Pérez García, A. M., Acosta-Pulido, J. A., \& Rodríguez Espinosa, J. M. 2007, AJ, 134, 2006

Rieke, G. H., Young, E. T., Engelbracht, C. W., et al. 2004, ApJS, 154, 25

Roche, P. F., \& Aitken, D. K. 1984, MNRAS, 208, 481

Rodriguez, C., Taylor, G. B., Zavala, R. T., et al. 2006, ApJ, 646, 49

Risaliti, G., Gilli, R., Maiolino, R., \& Salvati, M. 2000, A\&A, 357, 13

Rush, B., Malkan, M. A., \& Edelson, R. A. 1996, ApJ, 473, 130

Sanders, D. B., Mazzarella, J. M., Kim, D.-C., Surace, J. A., \& Soifer, B. T. 2003, AJ, 126, 1607 (RBGS)

Sanders, D. B., \& Mirabel, I. F. 1996, ARA\&A, 34, 749

Sanders, D. B., Scoville, N. Z., Young, J. S., et al. 1986, ApJ, 305, L45

Sanders, D. B., Soifer, B. T., Elias, J. H., et al. 1988a, ApJ, 325, 74

Sanders, D. B., Soifer, B. T., Elias, J. H., et al. 1988b, ApJ, 328, L35

Schlegel, D. J., Finkbeiner, D. P., \& Davis, M. 1998, ApJ, 500, 525

Schweizer, F. 2000, Phil. Trans. R. Soc. A, 358, 2063

Schweizer, F., \& Seitzer, P. 2007, AJ, 133, 2132

Skrutskie, M. F., Cutri, R. M., Stiening, R., et al. 2006, AJ, 131, 1163

Smith, J. D. T., Armus, L., Dale, D. A., et al. 2007, PASP, 119, 1133

Smith, J. D. T., Draine, B. T., Dale, D. A., et al. 2007, ApJ, 656, 770

Solomon, P. M., \& Vanden Bout, P. A. 2005, ARA\&A, 43, 677

Spoon, H. W. W., Armus, L., Cami, J., et al. 2004, ApJS, 154, 184

Spoon, H. W. W., Marshall, J. A., Houck, J. R., et al. 2007, ApJ, 654, L49

Stickel, M., Lemke, D., Klaas, U., et al. 2004, A\&A, 422, 39

Sturm, E., Rupke, D., Contursi, A., et al. 2006, ApJ, 653, L13

Su, K. Y. L., Kelly, D. M., Latter, W. B., et al. 2004, ApJS, 154, 302

Surace, J. A., Sanders, D. B., Vacca, W. D., Veilleux, S., \& Mazzarella, J. M. 1998, ApJ, 492, 116

Tacconi, L. J., Genzel, R., Tecza, M., et al. 1999, Ap\&SS, 266, 157

Tadhunter, C. 2008, New Astron. Rev., 52, 227

Thean, A. H. C., Gillibrand, T. I., Pedlar, A., \& Kukula, M. J. 2001, MNRAS, 327,369

Thompson, T. A., Quataert, E., \& Murray, N. 2005, ApJ, 630, 167

U, V., Sanders, D. B., Mazzarella, J. M., et al. 2012, ApJS, in press (arXiv:1209.1611)

Ueda, Y., Ishisaki, Y., Takahashi, T., et al. 2005, ApJS, 161, 185

Veilleux, S. 2006, New Astron. Rev., 50, 701

Veilleux, S., Cecil, G., \& Bland-Hawthorn, J. 2005, ARA\&A, 43, 769

Veilleux, S., Kim, D.-C., \& Sanders, D. B. 1999, ApJ, 522, 113

Veilleux, S., Kim, D.-C., Sanders, D. B., Mazzarella, J. M., \& Soifer, B. T 1995, ApJS, 98, 171

Veilleux, S., Rupke, D. S. N., Kim, D.-C., et al. 2009, ApJS, 182, 628

Wang, J., Heckman, T. M., Weaver, K. A., \& Armus, L. 1997, ApJ, 474, 659

Whitmore, B. C., Chandar, R., \& Fall, S. M. 2007, AJ, 133, 1067

Whitmore, B. C., Chandar, R., Schweizer, F., et al. 2010, AJ, 140, 75

Whitmore, B. C., Zhang, Q., Leitherer, C., et al. 1999, AJ, 118, 1551

Wrobel, J. M., \& Laor, A. 2009, ApJ, 699, L22

Xu, C. K., Domingue, D., Cheng, Y.-W., et al. 2010, ApJ, 713, 330

Young, J. S., Schloerb, F. P., Kenney, J. D., \& Lord, S. D. 1986, ApJ, 304, 443

Yuan, T.-T., Kewley, L. J., \& Sanders, D. B. 2010, ApJ, 709, 884

Yun, M. S., Reddy, N. A., \& Condon, J. J. 2001, ApJ, 554, 803

Yun, M. S., Scoville, N. Z., \& Knop, R. A. 1994, ApJ, 430, L109

Zhu, M., Gao, Y., Seaquist, E. R., \& Dunne, L. 2007, AJ, 134, 118

Zhu, M., Seaquist, E. R., \& Kuno, N. 2003, ApJ, 588, 243 